\title{
SIMULATION AND MODELING OF UNDERWATER ACOUSTIC COMMUNICATION CHANNELS WITH WIDE BAND ATTENUATION AND AMBIENT NOISE
}

\author{
by \\ Jie Huang \\ A thesis submitted to \\ the Faculty of Graduate Studies and Research \\ in partial fulfillment of \\ the requirements for the degree of \\ MASTER OF SCIENCE \\ School of Computer Science \\ at \\ CARLETON UNIVERSITY \\ Ottawa, Ontario \\ September, 2015
}

(c) Copyright by Jie Huang, 2015 


\section{Table of Contents}

List of Tables $\quad$ v

List of Figures $\quad$ vi

Glossary $\quad$ ix

Abstract $\quad$ xi

Acknowledgements xii

Chapter 1 Introduction 1

1.1 Statement of the Problem . . . . . . . . . . . . . . 1

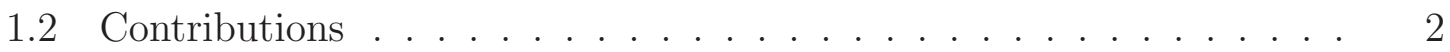

1.3 Overview of Results and Conclusions . . . . . . . . . . . . 3

1.4 Organization of Thesis . . . . . . . . . . . . . . 4

$\begin{array}{lll}\text { Chapter } 2 & \text { Background } & 6\end{array}$

2.1 Introduction . . . . . . . . . . . . . . . . . 6

2.2 Acoustic Waves . . . . . . . . . . . . . . . . . 7

2.2.1 Frequency ....................... 7

2.2.2 Acoustic Pressure . . . . . . . . . . . . . . 7

2.2.3 Velocity .................... 8

2.2.4 Energy ...................... 8

2.3 Multipath ............................. 9

2.4 Transmission Loss . . . . . . . . . . . . . . . . . . . . . . . . . 12

2.5 Noise . . . . . . . . . . . . . . . . . . . . . . . 14

2.6 Modulation Techniques . . . . . . . . . . . . . 15

2.6.1 Frequency Shift Keying . . . . . . . . . . . . . . 16

2.6.2 Phase Shift Keying . . . . . . . . . . . . . . . . . . . . 19 
2.7 BELLHOP Software . . . . . . . . . . . . . . . . . . . . . . . 19

Chapter 3 Related Work: State of the Art 23

3.1 Introduction . . . . . . . . . . . . . . . . . . . . 23

3.2 Wave Equation Models . . . . . . . . . . . . . . . . 23

3.3 Statistic Models . . . . . . . . . . . . . . . 26

$\begin{array}{lll}\text { Chapter } 4 & \text { Time Domain Modeling } & 28\end{array}$

4.1 Baseline . . . . . . . . . . . . . . . . . . . . 30

4.2 Multipath-Pressure-Sum . . . . . . . . . . . . . . . . 31

Chapter $5 \quad$ Frequency Domain Modeling 34

5.1 Incoherent Attenuation . . . . . . . . . . . . . . . . 37

5.1.1 Multi-Frequency-Merge-Path . . . . . . . . . . . . . 37

5.1.2 Multi-Frequency-Pressure-Sum . . . . . . . . . . . . . 38

5.1.3 Multi-Frequency-Merge-Path Down Conversion . . . . . . . . 39

5.1.4 Linear Fitting . . . . . . . . . . . . . . . . . . . . 44

5.2 Coherent Attenuation . . . . . . . . . . . . . . 46

5.2.1 Coherent Multi-Frequency-Merge-Path . . . . . . . . . . . 46

5.2.2 Coherent Multi-Frequency-Pressure-Sum . . . . . . . . . . . . 47

$\begin{array}{lll}\text { Chapter } 6 & \text { Noise Modeling and Simulation } & 49\end{array}$

$\begin{array}{lll}\text { Chapter } 7 & \text { Simulation Results } & 51\end{array}$

7.1 Time Domain Simulation Results . . . . . . . . . . . . . . 52

7.1.1 Baseline ........................ 52

7.1.2 Multipath-Pressure-Sum . . . . . . . . . . . . . 52

7.2 Frequency Domain Simulation Results . . . . . . . . . . . . . 56

7.2.1 Incoherent Simulation Result . . . . . . . . . . . 56

7.2 .2 Coherent ....................... 64

7.3 Comparison With the Experimental Data . . . . . . . . . . . 68

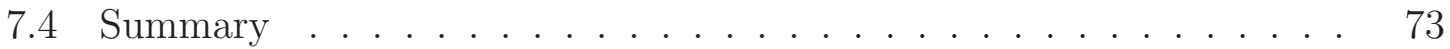


$\begin{array}{lll}\text { Chapter } 8 & \text { Conclusions and Future Work } & \mathbf{7 5}\end{array}$

8.1 Conclusions . . . . . . . . . . . . . . . . . . . . . 75

8.2 Future Work . . . . . . . . . . . . . . . . . . . 76

$\begin{array}{lll}\text { Appendix A Experiment environment } & 78\end{array}$

Appendix B Incoherent, coherent and acoustic pressure calculation $\begin{array}{lr}\text { interface in BELLHOP } & 80\end{array}$

B.1 Incoherent Interface . . . . . . . . . . . . . . . . . . . . . 80

B.2 Coherent Interface . . . . . . . . . . . . . . . . . . . . . 81

B.3 Acoustic Pressure Interface . . . . . . . . . . . . . . . . . . . 81

$\begin{array}{ll}\text { Bibliography } & 82\end{array}$ 


\section{List of Tables}

Table 5.1 Time complexities of all models. . . . . . . . . . . . . . . . . 48

Table A.1 Key Parameters . . . . . . . . . . . . . . . . . 78

Table A.2 Environmental file . . . . . . . . . . . . . . . . . . . . 79

Table B.1 Run-type parameters . . . . . . . . . . . . . . . 80 


\section{List of Figures}

Figure 1.1 Proposed underwater acoustic communication channel models. 2

Figure 2.1 An example of multipath propagation with reflection and refraction. . . . . . . . . . . . . . . . 10

Figure 2.2 Sound velocity profile as a function of depth. . . . . . . . . . . 11

Figure 2.3 Sound absorption coefficient in sea water. . . . . . . . . . . . 13

Figure 2.4 Four noise PSD and the total PSD. . . . . . . . . . . . . . . 15

Figure 2.5 An example of BFSK [57]. . . . . . . . . . . . . . . . . . 17

Figure 2.6 Theoretical BER curve of BFSK and 4-FSK. . . . . . . . . . . 18

Figure 2.7 An example of BPSK. . . . . . . . . . . . . . . . . . . . . 20

Figure 2.8 Theoretical BER curves of PSK. . . . . . . . . . . . . . . . . . 21

Figure 3.1 Sound propagation models. . . . . . . . . . . . . . . . . 24

Figure 3.2 The wavefronts of a plane wave. . . . . . . . . . . . . . . . . . 24

Figure 4.1 Underwater acoustic communication model. . . . . . . . . . . 28

Figure 4.2 Underwater channel simulator. . . . . . . . . . . . . . . . . . . 29

Figure 5.1 Spectrum of an analog signal with bandwidth 2 kilohertz after going through a bandpass filter. . . . . . . . . . . . 35

Figure 5.2 Spectrum of a signal after BPSK modulation. . . . . . . . . . 36

Figure 5.3 Half spectrum before and after attenuation ( $f_{c}$ is 20 kilohertz). 41

Figure 5.4 Half spectrum before and after attenuation with down conver-

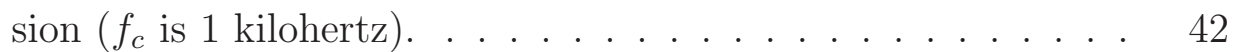

Figure 5.5 BER of the no down conversion - down conversion comparison. 43

Figure 5.6 Attenuation as a function of frequency and distance. . . . . . 45

Figure 5.7 Attenuation of linear fitting. . . . . . . . . . . . . . 45

Figure 7.1 Theoretical BER of BPSK modulation vs. the BER of the baseline model. . . . . . . . . . . . . . . . . . . 53 
Figure 7.2 Theoretical BER of BPSK modulation vs. the BER of the multipath-pressure-sum model. . . . . . . . . . . . . 54

Figure 7.3 The BER of the baseline model vs. the BER of the multipathpressure-sum model. . . . . . . . . . . . . . 55

Figure 7.4 Theoretical BER of BPSK modulation vs. the BER of the multi-frequency-merge-path model. . . . . . . . . . . . 57

Figure 7.5 The BER of the baseline model vs. the BER of the multifrequency-merge-path model. . . . . . . . . . . . . . 58

Figure 7.6 Theoretical BER of BPSK modulation vs. the BER of the multi-frequency-pressure-sum model. . . . . . . . . . . . 59

Figure 7.7 The BER of the baseline model vs. the BER of the multifrequency-pressure-sum model. . . . . . . . . . . . . . . 60

Figure 7.8 Theoretical BER of BPSK modulation vs. the BER of the multi-frequency-merge-path down conversion model. . . . . . . 61

Figure 7.9 The BER of the baseline model vs. the BER of the multifrequency-merge-path down conversion model. . . . . . . . . . 62

Figure 7.10 Theoretical BER of BPSK modulation vs. the BER of the linear fitting model. . . . . . . . . . . . . . . .

Figure 7.11 The BER of the baseline model vs. the BER of the linear fitting model. . . . . . . . . . . . . . . . . . . . . . . . 64

Figure 7.12 Theoretical BER of BPSK modulation vs. the BER of the coherent multi-frequency-merge-path model. . . . . . . . . .

Figure 7.13 The BER of the baseline model vs. the BER of the coherent multi-frequency-merge-path model. . . . . . . . . . . . 66

Figure 7.14 Theoretical BER of BPSK modulation vs. the BER of the coherent multi-frequency-pressure-sum model. . . . . . . . . 67

Figure 7.15 The BER of the baseline model vs. the BER of the coherent multi-frequency-pressure-sum model. . . . . . . . . . . . .

Figure 7.16 The BER of the coherent multi-frequency-pressure-sum model with colored noise. . . . . . . . . . . . . . . . 70 
Figure 7.17 The BER comparison of experimental data vs. the coherent multi-frequency-pressure-sum model. . . . . . . . . . . . 72 


\title{
Glossary
}

\author{
AWGN Additional white Gaussian noise \\ BER Bit error rate \\ BFSK Binary frequency shift keying \\ BPSK Binary Phase shift keying \\ FD Finite difference \\ FFP Fast Field Program \\ FFT Fast Fourier transform \\ FSK Frequency shift keying \\ ISI Inter Symbol Interference \\ MAPE Mean absolute percentage error \\ MFSK M-ary frequency shift keying \\ MSE Mean square error \\ NM Normal Mode \\ OFDM Orthogonal Frequency Division Multiplexing \\ PE Parabolic Equation \\ PSD Power spectral density \\ PSK Phase shift keying \\ RAP Reliable Acoustic Path \\ RMS Root mean square
}


SOFAR Sound fixing and ranging

TL Transmission loss

UWA Underwater Acoustic 


\section{Abstract}

Underwater acoustic communication uses acoustic waves to transmit and receive data under the water. Underwater radio waves suffer from high attenuation. Optical waves suffer from heavy scattering. Acoustic waves are a better communication mode for underwater environments. Simulation of underwater acoustic communication is challenging due to impairments, including attenuation, multipath propagation, noise and Doppler spread. In this thesis, several models are proposed and compared considering multipath attenuation and ambient noise. These models are divided into two groups. One group is in the time domain and the other is in the frequency domain. The models in the time domain and frequency domain all take into account the attenuation calculation along with white and colored noise. In the time domain, there are two models for attenuation. In the frequency domain, not only incoherent but also coherent attenuation are implemented. Incoherent means that the interference created by these phase differences is ignored. Coherent refers that the phase differences of signals propagating through different paths are taken into account. White noise is modeled as traditional Additive white Gaussian noise. Colored noise makes the white noise go through a low-pass filter with a profile similar to the one of the underwater ambient noise. The bit error rate versus energy per bit to noise power spectral density ratio $E_{b} / N_{0}$ is applied for evaluation. The simulations are implemented using MATLAB and the BELLHOP tracing program. The main conclusions of this work are:

1. In the situation where there is only one path from a transmitter to a receiver, the incoherent model can be used. The linear fitting model is the best one, due to the fact that it balances accuracy and efficiency.

2. In the situation with multipath propagation, the coherent model with colored noise in frequency domain is more similar to channels in the real shallow ocean environment. 


\section{Acknowledgements}

I have had the pleasure to meet a lot of friends during my study as a master student. I would like to thank all of you for your encouraging and supporting and making all this possible. Firstly, I would like to thank my supervisor Professor Michel Barbeau for patiently passing on knowledge and giving a lot of directions. I acknowledge financial support from Natural Sciences and Engineering Research Council (NSERC) of Canada (Engage Grant).

I also would like to thank the expert Craig Hamm, Martin Taillefer from Maritime Way Scientific Ltd and Stephane Blouin from DRDC Atlantic Research Center for the professional suggestions. Furthermore, I would like to thank all schoolfellows for the interesting discussions. Especially, I would like to thank Bita Hasannezhad and Inam Haq.

At last, I would like to appreciate the support from my family. I thank you all for the support and encouragement, my husband, my daughter, my mother, sister and of course my mother in-law and farther in-law. 


\section{Chapter 1}

\section{Introduction}

This chapter gives a brief introduction to the thesis. Section 1.1 presents the problems. Section 1.2 introduces the contributions. The overview of the simulation results are presented in Section 1.3. Section 1.4 describes the organization of the thesis.

\subsection{Statement of the Problem}

Underwater acoustic communications are widely used in military and civilian applications [14], such as ocean pollution monitoring, oil discovering, environmental data collection and disaster prediction. There are some differences between underwater and air transmission. Underwater communications have unique characteristics. The major characteristics consist of multipath propagation, attenuation and noise. Because of reflection and refraction, multipath propagation implies that the signal transmits through not only one path. Reflection happens at the sea surface and bottom. Refraction is caused by changes of sound velocity. Underwater sound velocity various because of salinity, water temperature and depth. Multipath propagation may result in severe interference among signals traveling through different paths. Attenuation provokes power loss during signal propagation under the water. Absorption loss and spreading loss are the two types of attenuation. Noise is another great source of communication errors, which includes ambient and localized noise.

Because of the special features of underwater transmission, new protocols and algorithms are designed. Evaluations and comparisons of new methods can be conducted through field experiments. However, they are expensive and time-consuming [45]. Effective evaluations and comparisons can be obtained through simulation. In this thesis, we study the problem of simulating underwater acoustic communications, considering multipath propagation, frequency dependent attenuation and ambient 


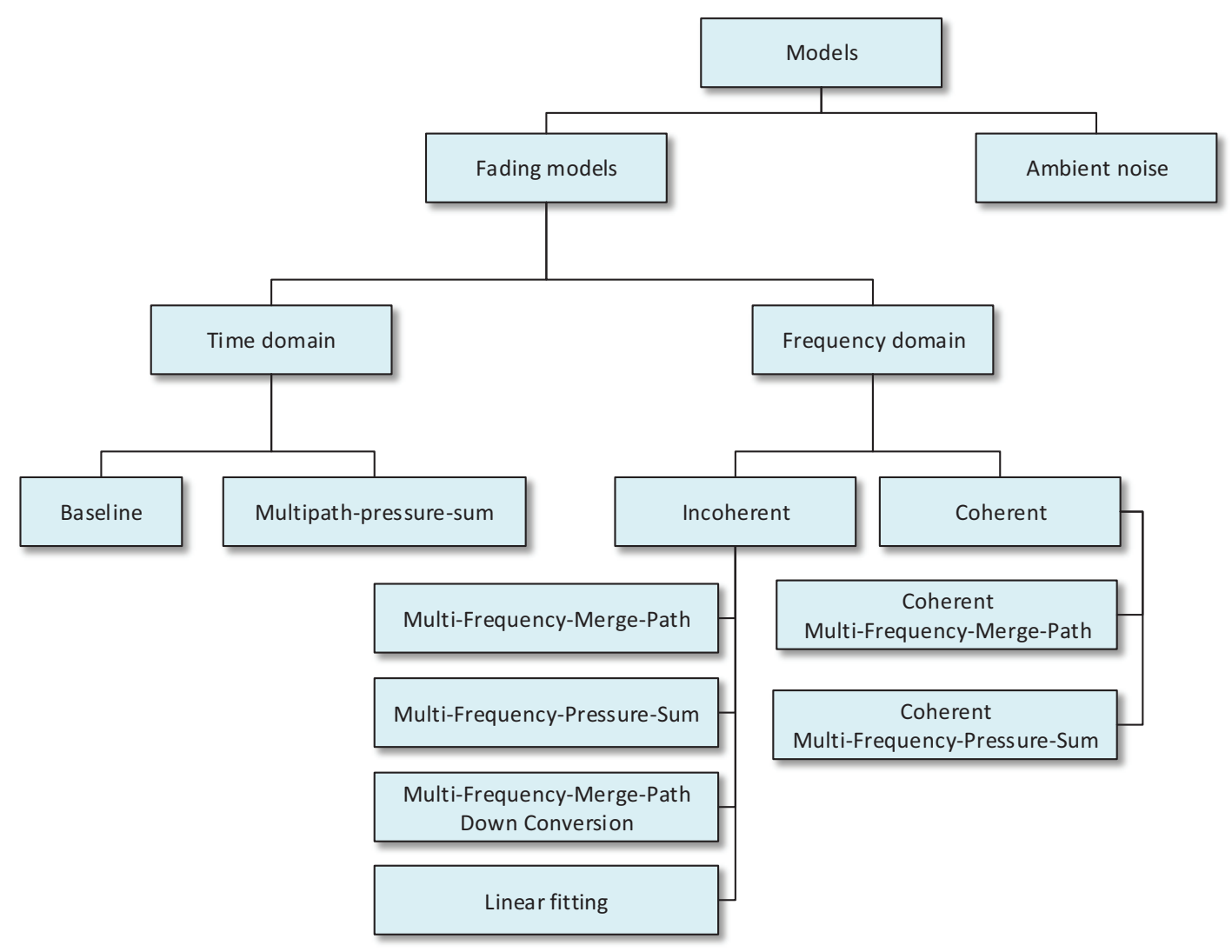

Figure 1.1: Proposed underwater acoustic communication channel models.

noise. The modeling and simulation of an underwater acoustic communication channel are challenging problems [10].

\subsection{Contributions}

In this thesis, eight underwater acoustic communication channel models are proposed, as shown in Figure 1.1. They are simulated and compared. The models are built in the time domain or in the frequency domain. Both incoherent and coherent attenuation are considered. Incoherent means that the interference created by phase differences is ignored. Coherent means that phase differences of signals propagating through different paths are taken into account. The first model, that we call baseline, comes from a book authored by Proakis [37]. The seven other models are our own extensions 
of this baseline model.

Two models are time domain representations. One is the baseline model and the other is the multipath-pressure-sum model, an extension of the baseline model. In the frequency domain, algorithms to calculate incoherent and coherent attenuation are put forward. Four models include algorithms for calculating incoherent attenuation: multi-frequency-merge-path, multi-frequency-pressure-sum, multi-frequencymerge-path down conversion and linear fitting. Two approaches calculate coherent attenuation: coherent multi-frequency-merge-path and coherent multi-frequencypressure-sum.

The simulations are implemented using the MATLAB and BELLHOP software. BELLHOP is a ray tracing tool used to calculate the eigenrays, acoustic pressures, delays and transmission loss. The acoustic pressures and delays computed by BELLHOP are transformed to attenuations in our models. For each model, a curve of bit error rate $(\mathrm{BER})$ versus energy per bit to noise power spectral density ratio $\left(E_{b} / N_{0}\right)$ is drawn to demonstrate the differences between the proposed models and theoretical reference or baseline model, with white noise or colored noise. A BER curve for the experimental data, acquired from a sea trial, is compared with the BER curves of all models. The BER curve of the coherent multi-frequency-pressure-sum model with colored noise is the most similar to the BER curve of the field experimental data. Statistical tests verify that these two BER curves have the same probability distribution.

\subsection{Overview of Results and Conclusions}

Eight underwater acoustic communication channel models are simulated, in the time domain or frequency domain models attenuated by incoherent or coherent loss with

white or colored noise. According to the simulation results, our main conclusions are:

1. All models, in time domain or in frequency domain, have higher BERs, i.e., worse performance, than the theoretical reference. It is due to the fact that the theoretical reference takes solely into account AWGN.

2. Similar, but not identical, simulation results can be obtained using incoherent 
models.

3. The BER values with all incoherent models are slightly larger than the BER values with the baseline model. All BERs of the coherent models are larger than the ones of the baseline model.

4. The models with coherent attenuation result in worse performance, in terms of BERs, than the models with incoherent attenuation.

5. Colored noise impacts the system performance more than white noise for all models, due to the frequency dependent feature of colored noise. For the incoherent models, when the $E_{b} / N_{0}$ ratio rises, the BERs with AWGN noise decrease more quickly than those with colored noise. However, for the coherent models with colored noise, the BER is falling almost the same as for the coherent models with white noise. The attenuation of the coherent models is larger than for call the incoherent models. The noise has less effects on the BERs in the coherent models than in the incoherent models.

6. The coherent attenuation is such that the BERs of coherent models cannot drop down to zero, even if the noise decreases to zero. The BERs of coherent models are maintained around a certain value after the $E_{b} / N_{0}$ ratio is higher than a threshold.

7. The BER curve of the coherent multi-frequency-pressure-sum model with colored noise is the most similar to the BER curve of the field experimental data. However, the BER curves are not identical because the environment are different from those of the field experiment. The statistical tests verify that these two BER curves have the same distribution.

\subsection{Organization of Thesis}

Chapter 2 introduces underwater acoustic communications, main impairments and modulation techniques.

Chapter 3 reviews the related works in the simulation of underwater acoustic communication. 
Chapter 4 explains the principles of two time domain models of an underwater acoustic communication channel.

Chapter 5 presents a general procedure of fading model in the frequency domain. Meanwhile, the principles of four incoherent models and two coherent models, in the frequency domain, are described.

Chapter 6 introduces two types of underwater noise models.

Chapter 7 presents the results obtained with the simulation of all underwater communication fading models discussed in Chapters 4 and 5 along with the noise models introduced in Chapter 6.

Chapter 8 shows the major conclusions and the future work. 


\section{Chapter 2}

\section{Background}

\subsection{Introduction}

About 71 percent of the surface on Earth is covered by the oceans. However, only up to 5 percent of it has been explored [1]. Hence, the utilization of the sea has attracted increasing attention during the recent decades. Underwater Acoustic Communication (UAC) refers to information transmission in water using acoustic waves [47]. It is a key technique of military or civilian underwater applications, such as ocean pollution monitoring, oil discovering, environmental data collection and disaster prediction.

Although several alternative waves, such as radio and optical waves, can be used for underwater communications, acoustic waves are the optimal solution [47]. Radio waves experience large absorption when traveling in water. The higher the frequency of waves, the larger absorption the signal suffers from. Therefore, the frequency range of usable radio waves is very low, from 30 Hertz to 300 Hertz. To transmit a signal underwater using radio waves at these frequencies, large aerials are needed and transmission power has to be high. Optical waves are not attenuated as much as radio waves. However, optical waves are subject to scattering. As a result, a highly precise pointing technique is required to transmit optical waves. Unfortunately, the precise pointing technology is not mature enough for application in practice. Therefore, acoustic waves are best for underwater transmission.

Nevertheless, communication through acoustic waves also face a certain degree of attenuation. Underwater acoustic communications are mainly influenced by path loss, noise, multipath, Doppler spread, and high and variable propagation delay [4]. We design the underwater acoustic communication models focusing on the impacts of multipath, transmission loss, delay and noise. This chapter explains these phenomenon one by one. Section 2.2 shows some fundamental concepts of acoustic waves. Section 2.3 introduces the concept and effects of multipath. Section 2.4 presents the concept 
and effects of underwater transmission loss. Section 2.5 shows the background of underwater noise. Two types of modulation technologies are introduced in Section 2.6. Section 2.7 introduces the BELLHOP software.

\subsection{Acoustic Waves}

\subsubsection{Frequency}

The vibrations of an acoustic signal are characterized by frequency $f$ (Hertz) or period time $T$ (second), where $f$ is equal to $1 / T[27]$. The frequency range of underwater acoustics is from 10 Hertz to one megahertz.

When the bandwidth is lower than one percent of the center frequency of a signal, the latter is narrow band [55]. When the bandwidth is between one to twenty percent of the center frequency of the signal, the latter is wide band. When the bandwidth is larger than twenty percent of the center frequency, the latter is ultra wide band. Although the absolute bandwidth of underwater communication is small, e.g., 2 kilohertz, the center frequency is also small, e.g., 20 kilohertz. Underwater acoustic communications is wide band because the center frequency is low relative to the signal bandwidth.

\subsubsection{Acoustic Pressure}

Acoustic pressure, or sound pressure, is the amplitude of an acoustic wave. It is the force of the sound on an area perpendicular to the propagation direction of the sound [18]. The Pascal (Pa) is the System International (SI) unit of acoustic pressure. One $\mathrm{Pa}$ is equal to one Newton per square meter $\left(\mathrm{N} / \mathrm{m}^{2}\right)$. Acoustic pressure is one of the most commonly used parameters in underwater acoustics [27]. It can be measured using a hydrophone.

The root mean square (RMS) is a square root of the mean of the sample square. Acoustic pressure level, or sound pressure level $(L)$, is the ratio of a sound pressure to a reference pressure. It is expressed on a logarithmic scale, in decibels (dB), as in the following equation [27]:

$$
L=20 \log _{10}\left(\frac{p}{p_{0}}\right) \mathrm{dB}
$$


$p$ is the root mean square acoustic pressure. $p_{0}$ is the reference acoustic pressure. Generally, the underwater reference pressure is one micropascal $(\mu \mathrm{Pa})$.

\subsubsection{Velocity}

The propagation of acoustic waves is due to the mechanical perturbation and elastic properties of water [27]. Velocity is the propagation speed of the perturbation. Normally, the acoustic velocity of sea water is about $1500 \mathrm{~m} / \mathrm{s}$. It is much lower than the speed of radio waves. The velocity changes, in the range of 1450 to $1550 \mathrm{~m} / \mathrm{s}$, according to water temperature, pressure and salinity [58]. Mackenzie summarized in an empirical equation for the sound velocity in ocean [28]:

$$
\begin{array}{r}
c(T, S, D)=A_{1}+A_{2} T+A_{3} T^{2}+A_{4} T^{3}+A_{5}(S-35) \\
+A_{6} D+A_{7} D^{2}+A_{8} T(S-35)+A_{9} T D^{3} \mathrm{~m} / \mathrm{s}
\end{array}
$$

Function $c$ is the sound velocity. Variable $T$ is temperature in Celsius. Variable $S$ is water salinity in parts per thousand. Variable $D$ is the depth in meters. $A_{1}$ to $A_{9}$ are constants with various values (see Ref. [28]).

\subsubsection{Energy}

When a signal propagates, acoustic energy is transmitted along paths [27]. Acoustic intensity and power are two characteristics of acoustic energy. Acoustic intensity $(I)$ is an averaged value of the energy per area, in Watts $/ \mathrm{m}^{2}$ :

$$
I=\frac{p^{2}}{2 \rho c} \text { Watts } / m^{2}
$$

Where $p$ is the pressure of an acoustic plane wave; $\rho$ is the density of the medium; $c$ is the sound velocity. Acoustic power $(P)$ is the acoustic intensity imposed on a surface $(S)$, in Watts. For an plane wave, we have:

$$
P=I \cdot S=\frac{p^{2} S}{2 \rho c} \text { Watts }
$$

When comparing the difference two powers $P_{1}$ and $P_{2}$, the following equation shows the relationship between acoustic power and pressure.

$$
10 \log _{10}\left(\frac{P_{1}}{P_{2}}\right)=20 \log _{10}\left(\frac{p_{1}}{p_{2}}\right)
$$


Where $p_{1}$ and $p_{2}$ are the sound pressures corresponding to the power level $P_{1}$ and $P_{2}$ respectively.

\subsection{Multipath}

Multipath means that a signal propagates from a transmitter to a receiver along several different paths [27]. Each path has its own departure angle and delay. Besides a quasi direct path, other paths come form the reflection at the sea surface and seabed along with the refraction caused by variable sound velocity. A path, or sound ray, from a transmitter to a receiver is called an eigenray [21]. The location of a transmitter and a receiver, sea depth and sound velocity profile are major parameters that determine the eigenrays.

Figure 2.1 shows an example of multipath propagation with reflection and refraction produced using the BELLHOP software [35]. In this example, the seabed is assumed to be muddy sandy. The sound velocity changes according to the Munk sound profile [41], see Figure 2.2. The blue (thin line) and black (thick line) straight lines are the eigenrays reflected by the sea surface, seabed or both. The red curves (with circles) are the eigenrays deformed by refraction.

Underwater multipath propagation results in fading and reverberation effects, which cause higher transmission loss [42]. The effects of multipath for transmission loss in shallow water are simpler than those in deep water. A transmission loss model of multipath propagation in shallow water has to consider the repeated reflection at sea surface and seabed. A propagation model in deep water has to take into account six major impacts: surface reflection, surface duct, seabed bounce, convergence zone, SOund Fixing And Ranging (SOFAR) channel and Reliable Acoustic Path (RAP). Surface duct is a phenomenon where a deep sound channel is present. Sound waves can only propagate inside that channel. Seabed bounce propagation means that a sound wave hits the seabed and reflects back to the surface. The convergence zone is a zone near the surface with high sound intensity. It is generated when the transmitter is located in shallow water while the receiver is in deep water. Moreover, the sound velocity in deep ocean is larger than that in shallow ocean. A SOFAR channel or deep sound channel is along the level of minimum sound velocity. A RAP is present when 


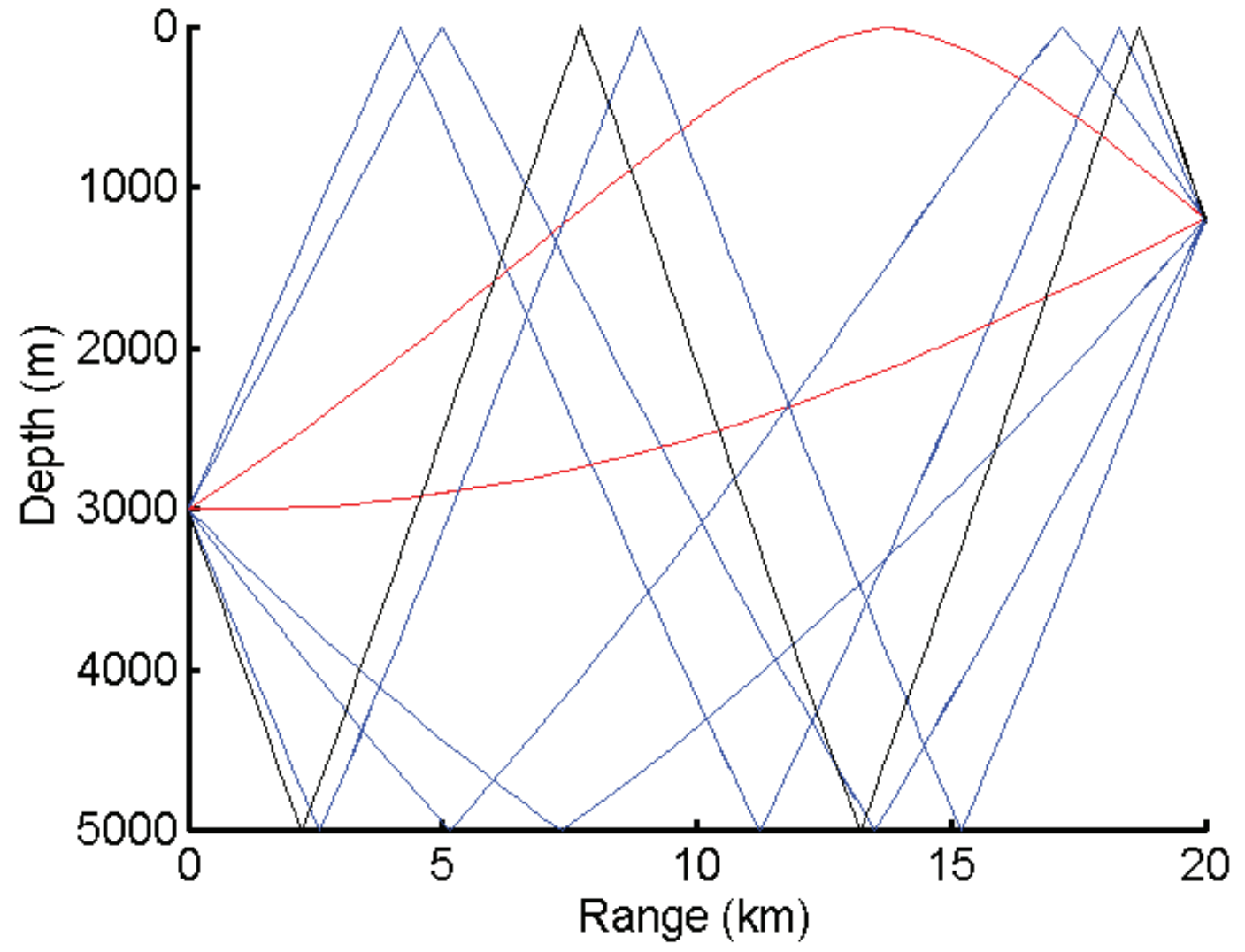

Figure 2.1: An example of multipath propagation with reflection and refraction. 


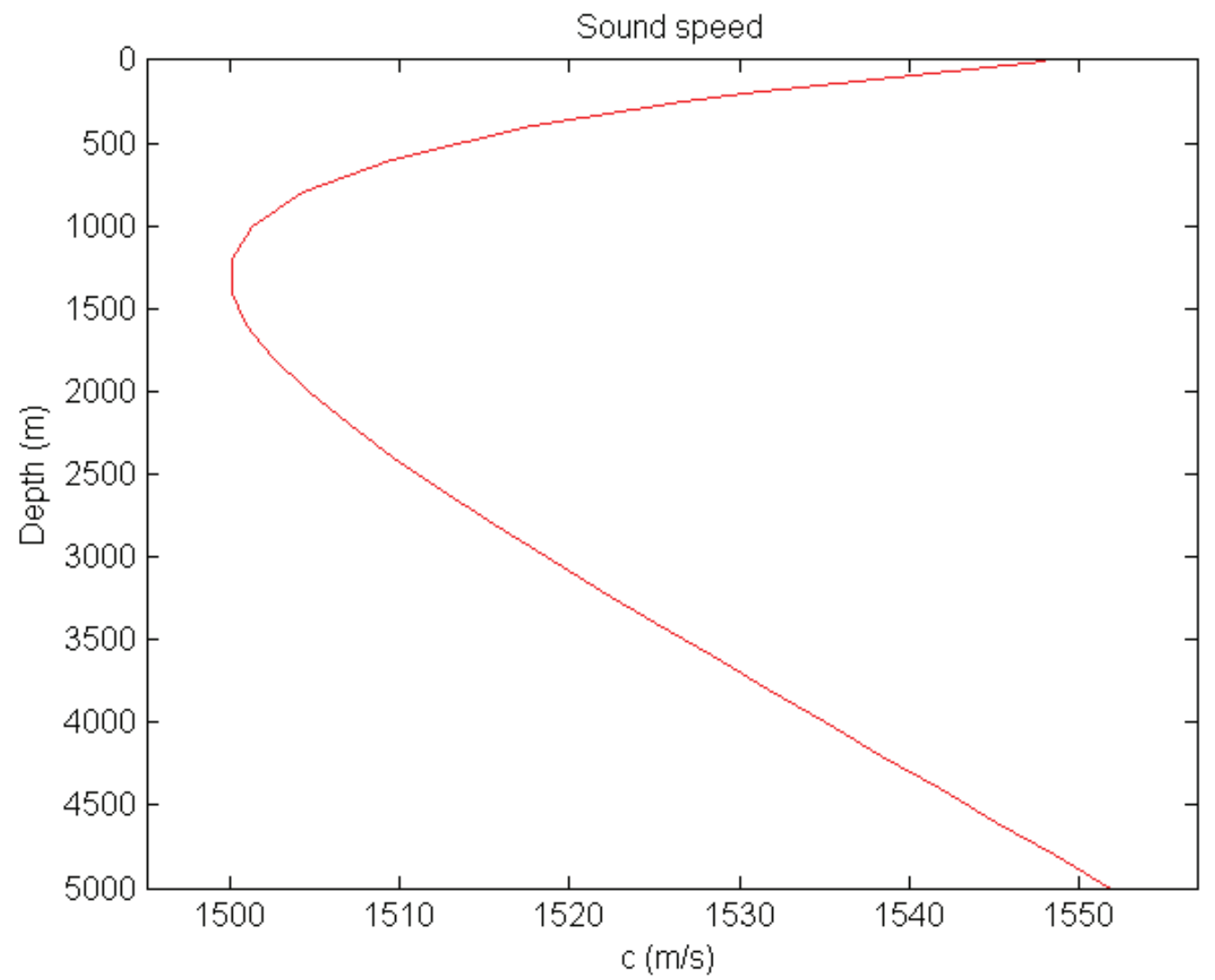

Figure 2.2: Sound velocity profile as a function of depth. 
a path has little interaction with the sea surface or seabed. The sound can propagate through a RAP at moderate distance when the transmitter is in deep water and the receiver lies in shallow water.

\subsection{Transmission Loss}

The transmission loss (TL) or propagation loss means the reduction of acoustic intensity from a reference to a destination [18]. Let $I_{0}$ be the intensity at a reference distance (one meter), $I$ be the intensity at distance $r(\mathrm{~m})$ and depth $d(\mathrm{~m}), p$ be the acoustic pressure $(\mu \mathrm{Pa})$ at distance $r$ and depth $d, p_{0}$ be the reference acoustic pressure $(\mu \mathrm{Pa})$. The transmission loss $T L$ can be represented as:

$$
\begin{aligned}
T L & =-10 \log \left(\frac{I(r, d)}{I_{0}}\right) \\
& =-20 \log \left(\frac{p(r, d)}{p_{0}}\right) \mathrm{dB}
\end{aligned}
$$

The transmission loss consists of spreading loss and absorption loss [27]. When an acoustic signal propagates through a surface, the acoustic intensity drops. The drop rate is inversely proportional to the distance. The intensity reduction of a homogeneous point source can be calculated as below.

$$
T L=20 \log \left(\frac{R}{R_{1}}\right) \mathrm{dB}
$$

$R$ is the distance between the transmitter and receiver in meters. $R_{1}$ is the reference distance.

Due to the viscosity of water and molecular relaxation, sea water absorbs the energy of the acoustic waves. This is called absorption loss. A large number of absorption models were proposed. The model proposed by Francois and Garrison in 1982 is the most commonly used model nowadays [27]. This model covers three parts: the boric acid contribution, magnesium sulphate contribution and pure water contribution, shown as the following equation:

$$
\alpha=A_{1} P_{1} \frac{f_{1} f^{2}}{f_{1}^{2}+f^{2}}+A_{2} P_{2} \frac{f_{2} f^{2}}{f_{2}^{2}+f^{2}}+A_{3} P_{3} f^{2} \quad \mathrm{~dB} / \mathrm{km}
$$

Variable $\alpha$ is the absorption. Coefficients $A_{1}$ to $A_{3}$ represent the impacts of sound speed and water temperature. Coefficients $P_{1}$ to $P_{3}$ capture the effects of depth. 


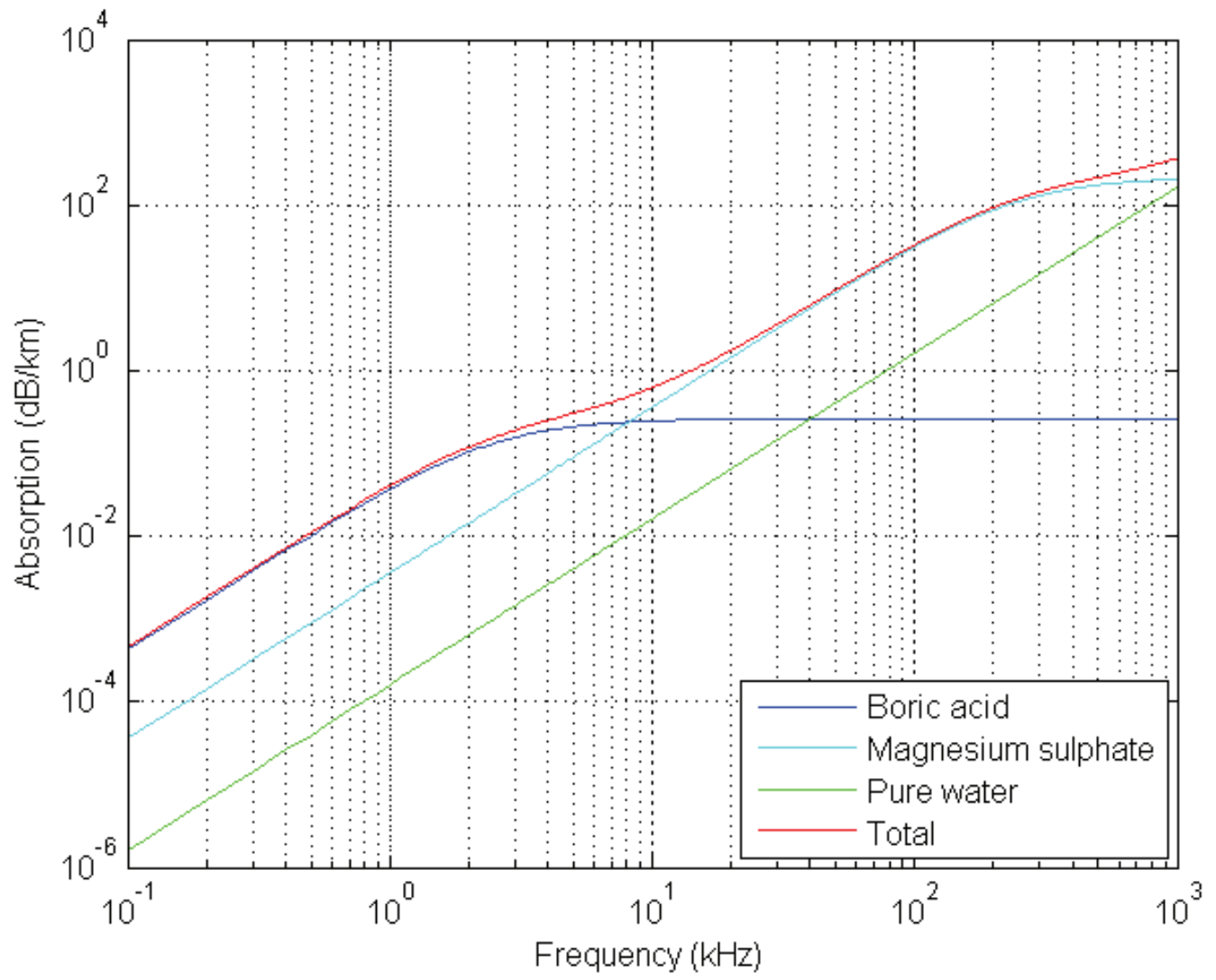

Figure 2.3: Sound absorption coefficient in sea water.

Coefficient $f_{1}$ is the relaxation frequency of boric acid. Coefficient $f_{2}$ is the relaxation frequency of magnesium sulphate. An analysis of the Francois-Garrison model was conducted by Ainslie and McColm. Figure 2.3 shows the contribution of the three parts in the Francois-Garrison model and total absorption as a function of frequency [2]. The conventional calculation of total transmission loss for spherical spreading consists of adding the spreading loss (Eq. 2.1) and absorption (Eq. 2.2) together, as in the following equation [27].

$$
T L=20 \log R+\alpha R
$$

Although this simple equation is approximate, it is enough for analyzing the underwater acoustic performance when there are no special requirements, such as the influence of bubbles, changing of sound velocity, multipath and Doppler effect [27]. 


\subsection{Noise}

Noise is unwanted signal in water. The sources of noise in the ocean are classified as ambient or localized [50]. Ambient noise is caused by shrimps, fishes, turbulence and various mammals, which always exists in the background of the sea. Localized noise is only present in certain areas. Many sources, such as rain, thermal and wave, create ambient noise which can be modeled by a Gaussian distribution [27].

The underwater ambient noise has four major sources: turbulence, waves, shipping and thermal [48]. The power spectral density (PSD) $N_{t}(f)$, in $\mathrm{dB}$ re $\mu \mathrm{Pa}$, as a function of frequency $f$, in kilohertz, for turbulence noise is modeled as:

$$
N_{t}(f)=17-30 \log f \mathrm{~dB} \text { re } \mu \mathrm{Pa}
$$

The unit $\mathrm{dB}$ re $\mu \mathrm{Pa}$ means a logarithmic measure of a acoustic pressure relative to a reference pressure of one $\mu \mathrm{Pa}$.

The power spectral density $N_{w}(f)$, in $\mathrm{dB}$ re $\mu \mathrm{Pa}$, as a function of frequency $f$, in kilohertz, with wind speed $w$, in $\mathrm{m} / \mathrm{s}$, for wave noise is modeled as:

$$
N_{w}(f)=50+7.5 w^{1 / 2}+20 \log f-40 \log (f+0.4) \mathrm{dB} \text { re } \mu \mathrm{Pa}
$$

The power spectral density $N_{s}(f)$, in $\mathrm{dB}$ re $\mu \mathrm{Pa}$, as a function of frequency $f$, in kilohertz, for shipping noise is modeled as:

$$
N_{s}(f)=40+20(s-0.5)+26 \log f-60 \log (f+0.03) \mathrm{dB} \text { re } \mu \mathrm{Pa}
$$

$s$ is a shipping activity factor in the range from zero to one, which is from low to high. $N_{t h}(f)$, in $\mathrm{dB}$ re $\mu \mathrm{Pa}$, as a function of frequency $f$ in kilohertz for thermal noise is modeled as:

$$
N_{t h}(f)=-15+20 \log f \mathrm{~dB} \text { re } \mu \mathrm{Pa}
$$

The total noise PSD is the sum of all noise sources in linear form:

$$
N_{T}(f)=N_{t}(f)+N_{w}(f)+N_{s}(f)+N_{t h}(f) \mu \mathrm{Pa}
$$

Figure 2.4 shows the curves of the PSD of turbulence, wave, shipping and thermal noise and the total PSD [42]. Wind speed $w$ is set to $10 \mathrm{~m} / \mathrm{s}$. Shipping activity factor 


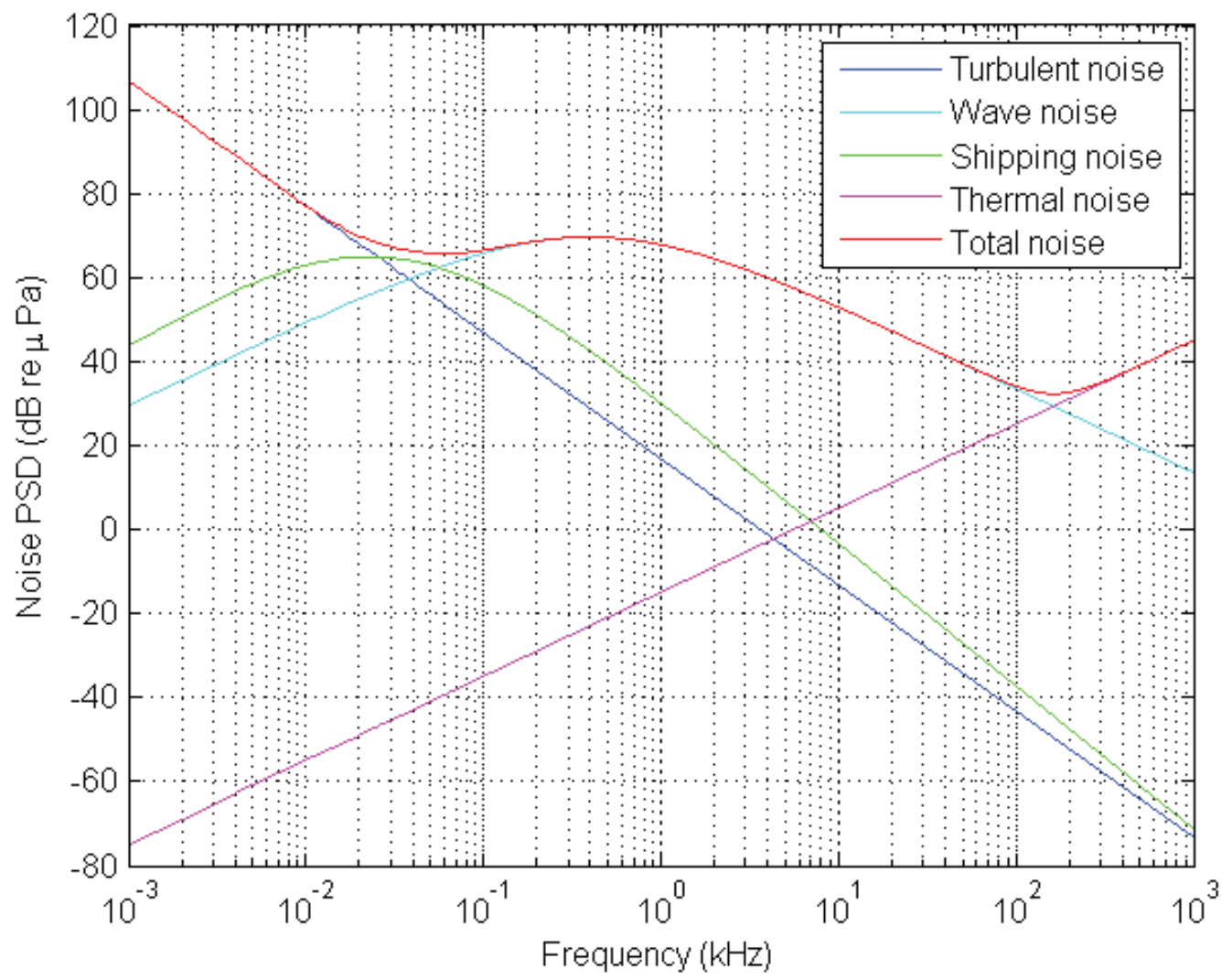

Figure 2.4: Four noise PSD and the total PSD.

$s$ is set to 0.05 . When the frequency is lower than $20 \mathrm{Hertz}$, turbulence noise plays the key role. When the frequency is from 20 to 80 Hertz, shipping noise plays the key role. When the frequency is from 80 Hertz to 100 kilohertz, waves driven noise plays the key role. When the frequency is larger than 100 kilohertz, thermal noise plays the key role.

\subsection{Modulation Techniques}

The characteristics of underwater acoustic communications, such as multipath propagation and phase variation, are the causes of limited data rate and low bandwidth efficiency [25]. To address these issues, adapted modulation techniques are required. 
Modulation refers to a process where properties of a carrier signal are changed according to the content of a message signal. The message signal can be restored using an inverse process, called demodulation. There are two digital modulation methods used for underwater acoustic communications: Frequency Shift Keying (FSK) and Phase Shift Keying (PSK) [56, 49].

\subsubsection{Frequency Shift Keying}

FSK is a digital modulation technique used by underwater devices. It is a scheme that consists of modifying the frequency of a carrier signal according to the content of a message signal [11]. Binary FSK (BFSK) and M-ary FSK (MFSK) are two variants of FSK. The $M$ in MFSK means the number of digital symbols used for modulation is larger than two. When the number of digital symbols equals to two, it is BFSK.

BFSK uses two signals, $s_{1}$ and $s_{2}$, at two different frequencies, $f_{1}$ and $f_{2}$, to represent 1 and 0, shown as the following equation [59].

$$
\begin{aligned}
& s_{1}(t)=A \cos \left(2 \pi f_{1} t+\phi_{1}\right), \text { for binary "1" } \\
& s_{2}(t)=A \cos \left(2 \pi f_{2} t+\phi_{2}\right), \text { for binary "0", } \quad k T \leq t \leq(k+1) T
\end{aligned}
$$

Where $\phi_{1}$ and $\phi_{2}$ are initial angles for respectively $s_{1}$ and $s_{2}$. $T$ is the bit period. $k=-\infty, \ldots,-1,0,1, \ldots, \infty$, is the index of the period.

Figure 2.5 shows an example of a BFSK signal in the time domain.

There are two types of FSK modulation: non-coherent and coherent. When the initial angles $\phi_{1}$ is different from $\phi_{2}$, the two modulated signals $s_{1}$ and $s_{2}$ are noncoherent. This type of BFSK is non-coherent FSK. When the initial angles $\phi_{1}$ and $\phi_{2}$ are the same, the type of BFSK is coherent. Non-coherent FSK works best for underwater acoustics communication, because it requires detection of energy instead of phase [4]. Phase tracking is challenging due to its variability in an underwater acoustic channel. In FSK modulation, the effects of multipath can be mitigated, and reverberation decreased, by adding time guards between consecutive pulses. The theoretical performance of FSK modulation can be modeled by a curve of bit error rate $(\mathrm{BER})$ versus energy per bit to noise power spectral density ratio $\left(E_{b} / N_{0}\right)$, as shown in Figure 2.6. Coherent FSK has slightly better BER than non-coherent FSK. 

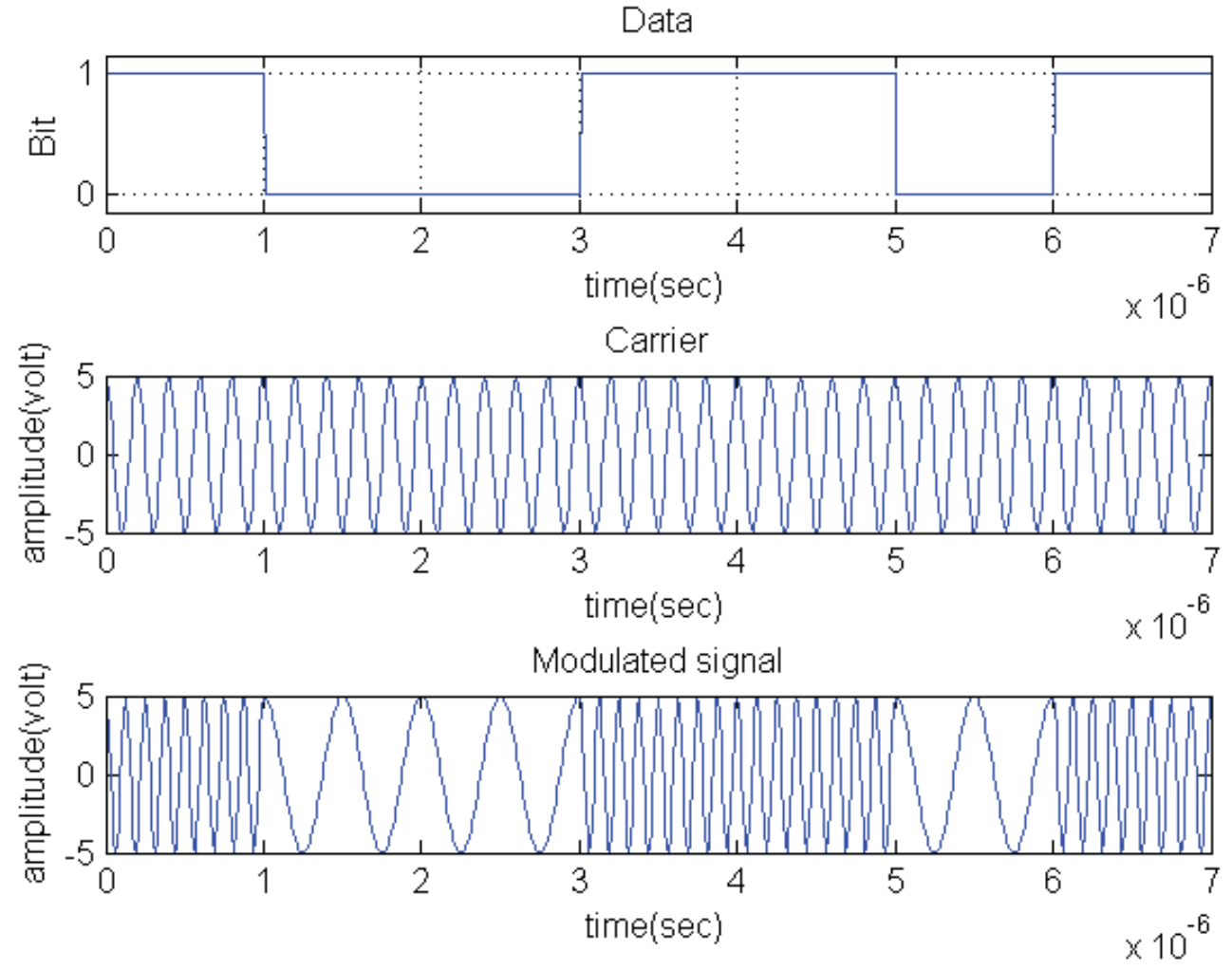

Figure 2.5: An example of BFSK [57]. 


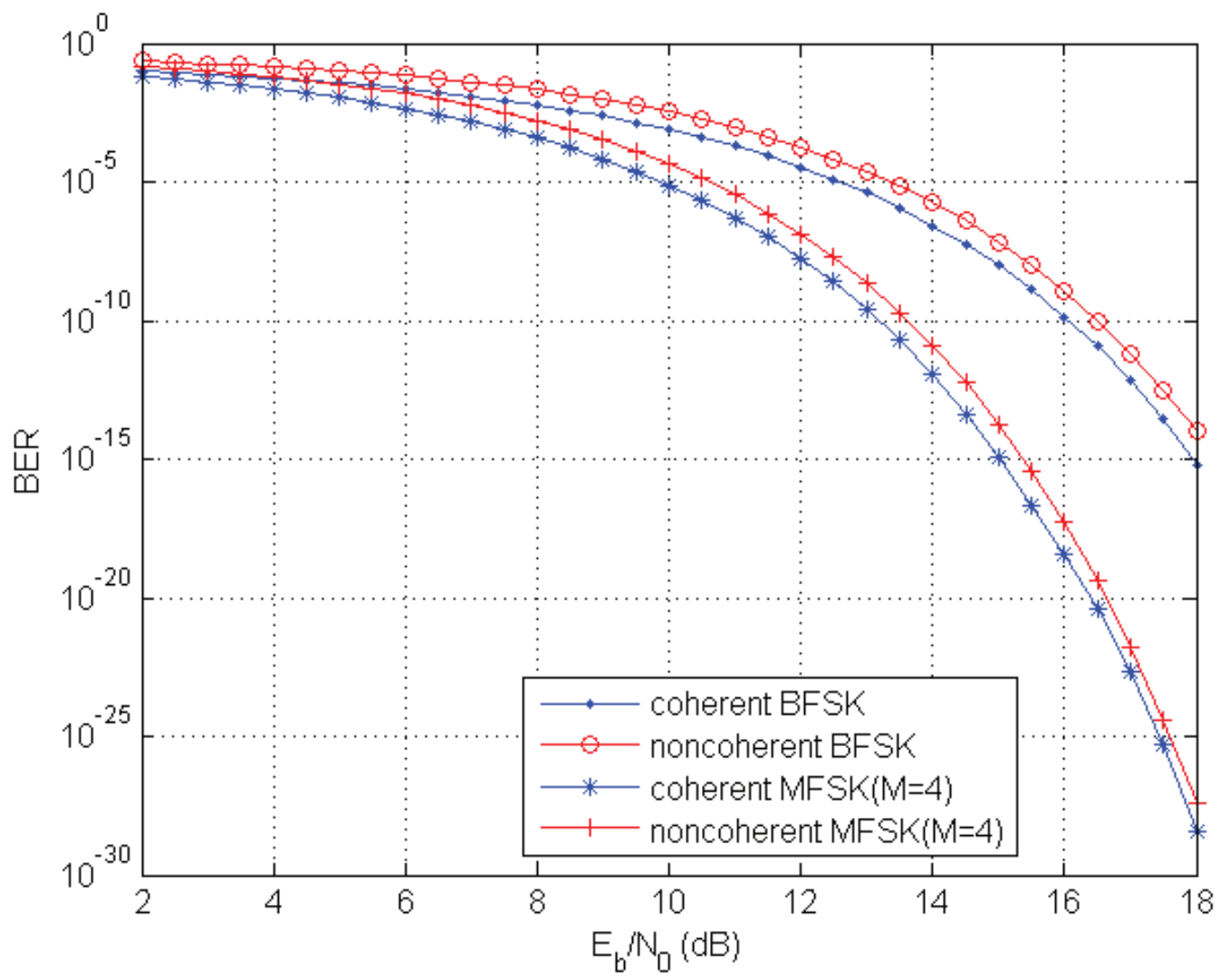

Figure 2.6: Theoretical BER curve of BFSK and 4-FSK. 
Non-coherent FSK modulation has high energy efficiency, but it has low bandwidth efficiency [4]. Therefore, fully coherent modulation techniques, such as Phase Shift Keying (PSK), emerged for high data rate and high throughput systems.

\subsubsection{Phase Shift Keying}

Phase Shift Keying (PSK) refers to a modulation scheme that modifies the phase of a carrier signal according to the content of a message signal [11]. Binary PSK (BPSK) uses two phases, 0-degree and 180-degree, to represent 1 and 0, shown as in the following equation [59]:

$$
\begin{aligned}
& s_{1}(t)=A \cos \left(2 \pi f_{c} t\right), \quad \text { for binary "1" } \\
& s_{2}(t)=-A \cos \left(2 \pi f_{c} t\right), \quad \text { for binary " } 0 ", \quad 0 \leq t \leq T
\end{aligned}
$$

$s_{1}$ and $s_{2}$ are two modulated signals as a function of time $t . f_{c}$ is the frequency of a carrier wave. $T$ is the bit period. After using BPSK, the signals are modulated to be 0 or 1 . An example is shown in Figure 2.7 This scheme is the simplest and most robust among all the PSK modulation techniques. It has the lowest bit error rate for the same $E_{b} / N_{0}$ ratio, as Figure 2.8 .

\subsection{BELLHOP Software}

BELLHOP is an open source ray tracing program coded in FORTRAN, MATLAB and PYTHON by Porter [41, 35]. Gaussian beam theory is the principle used to calculate acoustic pressure. The overview of the structure of the BELLHOP software see Ref. [35]).

An environmental file, with the .env extension, is the input to the BELLHOP software [35]. Input information mainly consists of locations of a source and a destination, a frequency and running parameters. Additional conditions can be provided with other files. For example, a sound speed profile, a file with the .ssp extension, can be provided when the sound speed changes depending on the depth. Moreover, a variable seabed can be specified with a bathymetry file. All the input information can be visualized using the plot programs of BELLHOP, such as plotssp, to plot the sound speed profile, and plotbty, to plot the seabed specified in a bathymetry file. 

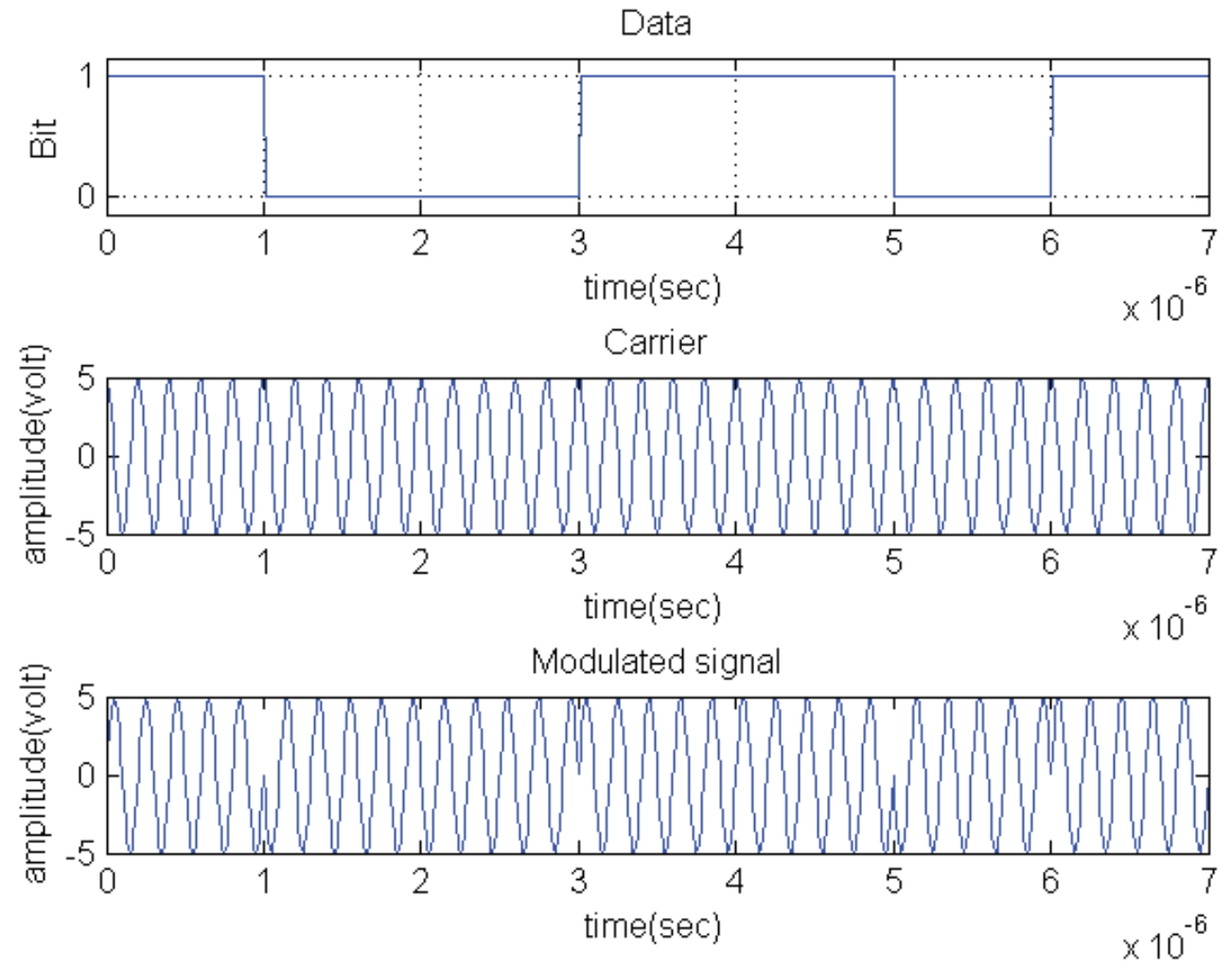

Figure 2.7: An example of BPSK. 


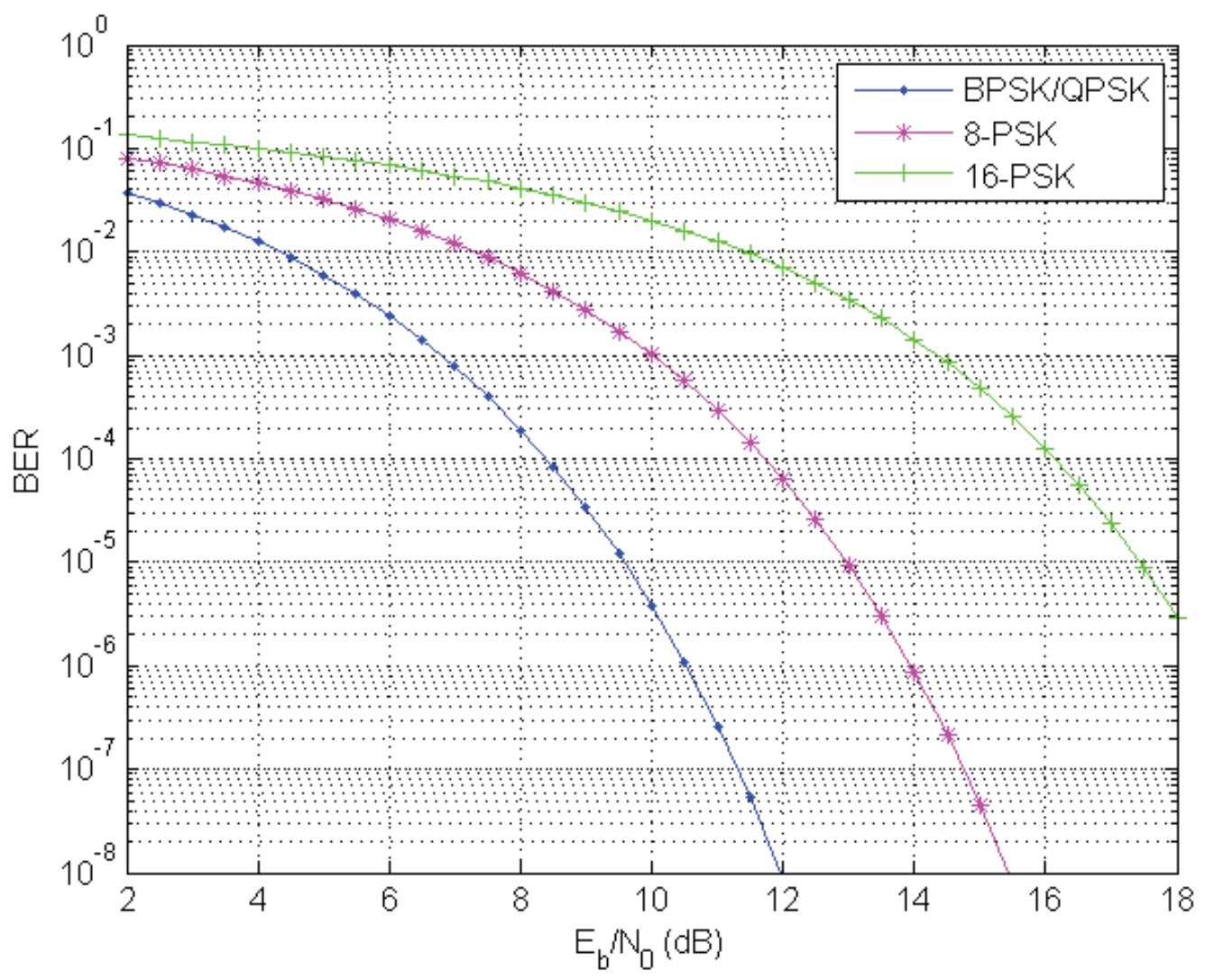

Figure 2.8: Theoretical BER curves of PSK. 
The BELLHOP software determines which function is invoked and what data is produced according to the running parameters contained in the environmental file. Output data include ray coordinates, eigenrays, acoustic pressure, travel time and transmission loss (coherent, incoherent or semi-coherent). The output files can be read and plotted using the BELLHOP functions plotray, plotshd, plottlr, plottld and plotarr. 


\section{Chapter 3}

\section{Related Work: State of the Art}

\subsection{Introduction}

Since underwater acoustic communications have been developed for a long time, several modeling and simulation techniques have been published. The aim of modeling and simulation is to predict the performance of underwater acoustic systems [14]. These simulation methods can be divided into two categories. The methods in the

first category are variants based on wave equation models to calculate multipath propagation loss. The methods in the second category apply statistic techniques. Section 3.2 describes the wave equation models. Section 3.3 presents the models using statistic techniques.

\subsection{Wave Equation Models}

Wave equation refers to a hyperbolic partial differential equation, which is used to describe sound waves [14]. There are five types of propagation models: Fast Field Program (FFP), Normal Mode (NM), ray model, Parabolic Equation (PE) model, and Finite-Difference (FD) solutions [23], see Figure 3.1. The FFP and NM model were initially used to solve problems with range independence. However, they can be extended to handle range dependent problems. The ray, PE and FD models are designed to treat range dependent issues.

The ray model calculates transmission loss by tracing rays. The assumption is that the rays that sound propagates along are normal to wavefront [19]. Wavefront is the surface where the wave propagates through at the same phase. For example, the wavefronts of a plane wave are also planes, see Figure 3.2. When a wave is generated by a point source with a fixed sound velocity, the sound rays modeling the propagation paths are straight lines. When the sound velocity changes, the rays are 


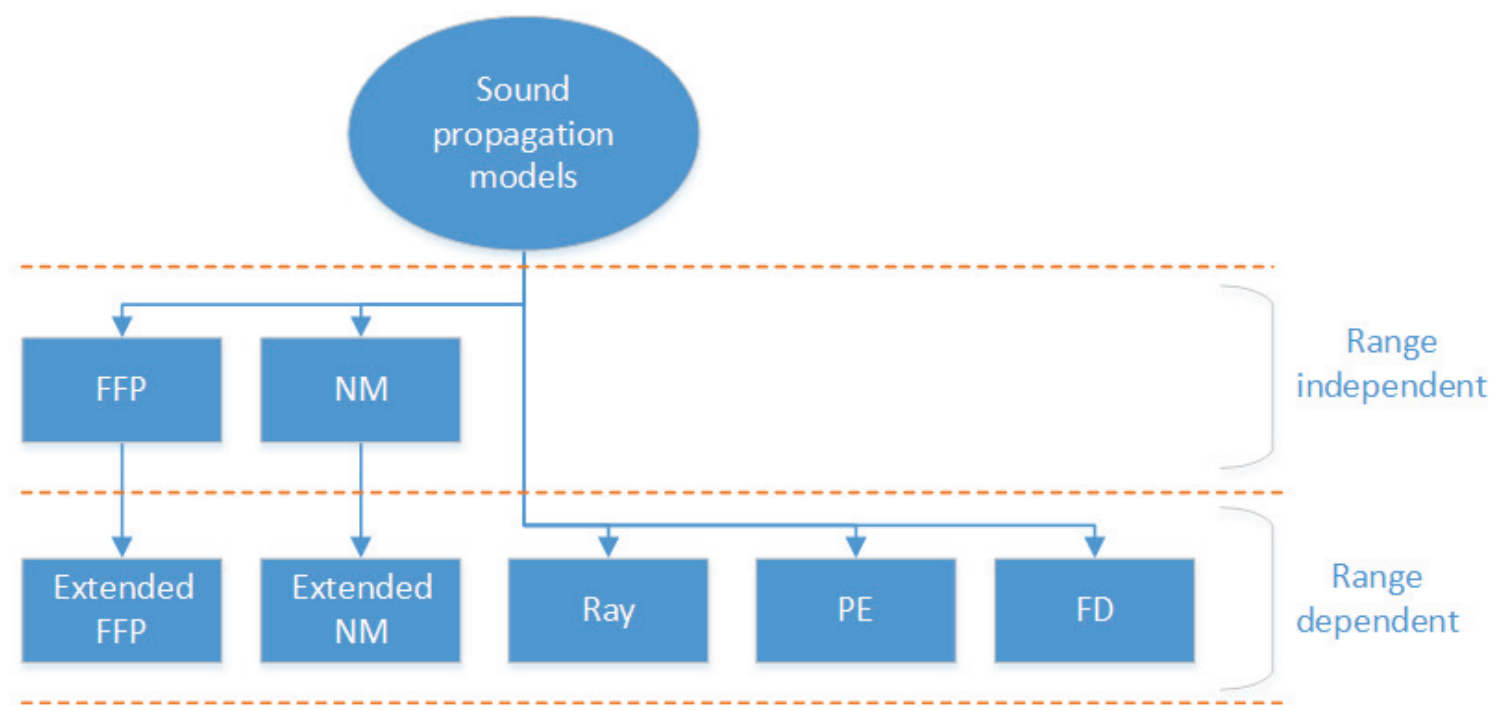

Figure 3.1: Sound propagation models.

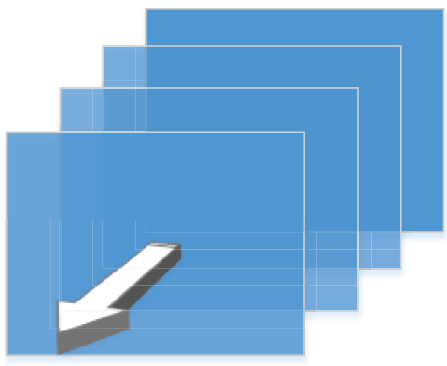

Wave propagation

Figure 3.2: The wavefronts of a plane wave. 
curved. The ray model is an approximation for high frequency acoustic waves. It calculates the TL for a certain receiver depth and range, aggregating all the paths. Since ray model is based on a geometrical acoustic approximation, it is limited to high frequency and ignores diffraction. The BELLHOP software uses the ray model with Gaussian beam tracing. A beam with a Gaussian intensity profile is connected with a ray. Other commonly used ray models include RAY [9], TRIMAIN [13] and Hamiltonian Acoustic Ray-tracing Program for the Ocean (HARPO) [24].

The NM model is in a integral form of the wave equation with a range independent assumption. The TL calculation is done at all depths and ranges of the receiver. The NM model has lower time complexity than the ray model. However, NM needs information about the sea bed structure, fluid density and various sound velocity in different layers of the fluid. Since the number of modes increases with frequency, the NM model is more suited to the low frequency applications [33]. Examples of models using NM include KRAKEN [34], Modal Acoustic Transmission Loss (MOATL) [20], SACLANTCEN Normal-mode Acoustic Propagation (SNAP) [22] and COUPLE [15]. Two solutions, coupled mode and adiabatic approximation, have been developed to extend NM from range independence to dependence [14]. Adiabatic approximation assumes that there is no energy exchange from one mode to the other. It is based on the work of Pierce to adapt the wave equation to range dependence [33]. Since it ignores the energy exchange, the calculation is simple and fast. However, it can be used only when the energy changes gradually. The coupled mode divides the range into several segments [23]. Each segment computes the TL using the standard NM. The Coupled mode is straightforward, but it results in high time complexity.

The FFP model is also an integration of the wave equation, like the NM model. The FFP model uses the same approach of NM to separate the parameters of the wave equation. Nevertheless, it applies a different Hankel function than in the NM model. Moreover, a Fast Fourier Transform (FFT) is used to evaluate the result of FFP. Similar to NM, a coupled FFP solution was published to solve range dependent problems [43]. In coupled FFP, the range dependent environment is divided into several range independent parts. Then, a boundary-element or finite-element method is combined. 
The PE model assumes that the speeds of energy propagation are similar to a reference speed [23]. Unlike the ray model, the PE model handles all the diffraction effects in the acoustic channel. Therefore, it is more suitable to lower frequencies than the ray model. The PE model was essentially developed to solve problems with a long range and small propagation angles (less than or equal to about 15 degrees) by Tappert [51]. Afterwards, it was modified by Thomson and Chapman for wide propagation angles [54]. The modified wide angle equation decreases the impact of phase error. The PE model can easily be extended into three dimensions (3D). The $\mathrm{PE} 3 \mathrm{D}$ model is normally combined with the wide angle method. A wide angle PE model in a 3D heterogeneous flowing liquid was designed by Godin [17]. It can achieve higher precision than the wide angle PE model.

The FD model is derived from the classic PE model [14]. It approximates the differential equation by finite discrete differences. The FD model includes explicit and implicit methods. The explicit method can be easily implemented, but it is unstable and inaccurate. Implicit method is stable but more time-consuming than explicit method. The FD model is one of the most pervasive forms for PE model. It has been applied to situation where the sea bottom is elastic and geometrically irregular.

\subsection{Statistic Models}

The models based on the wave equation are limited to one frequency calculation in a certain underwater geometry. For multiple frequencies, the models have to be repeated. Statistical models make the acoustic channel simulation more efficient. For the past few years, there are increasing research on the development of statistical acoustic channel models. According to the analyses of experimental acoustic data, different statistical laws have been used, such as Rician fading, Rayleigh fading, lognormal distribution and K-distribution [38]. Different underwater environments and acoustic signals cause statistical model changes.

Galvin and Wang implemented experiments to build underwater acoustic communication using pulse carrier signals [16]. The center frequency of their acoustic signal is five kilohertz and bandwidth is two kilohertz. They analyzed a multipath channel and 
a single path channel. The amplitude fluctuation shows a Rician distribution. The phase fluctuation follows a Gaussian distribution. Duncan et al. proposed a Rayleigh distribution method for the coherent sound pressure calculation in long range shallow water [12]. The center frequency of their acoustic signal is seven kilohertz. Some experiments were conducted according to coherent and incoherent pressures obtained by the BELLHOP software. The coherent pressure was normalized by being divided by the incoherent pressure. Then, it was converted to decibels to give normalized coherent received levels. Histograms of normalized coherent received levels for every range intervals of 100 meters compared to the expected Rayleigh distribution showed good fit. Yang et al. found statistical characteristics for high frequency (from 15 to 19 kilohertz) acoustic channels [60]. They stated that the statistics of long term fading are different from those of short term fading. Short term means that the high frequency signal goes over a short time. The major cause of fading is the turbulence. The short term fading was found following the Rayleigh distribution. Long term means that the high frequency signal goes over a long time. The major cause of fading comes from the fine structure perturbation. The long term fading was found following the log-normal distribution. Sequentially, they combined the two statistics together and concluded that the high frequency fading follows the K-distribution. 


\section{Chapter 4}

\section{Time Domain Modeling}

In this chapter, a model of underwater acoustic communication is built. It includes three parts: a Binary Phase Shift Keying (BPSK) modulator, an underwater acoustic communication channel simulator and a BPSK demodulator, shown in Figure 4.1. Function $s(t)$ is the amplitude $(\mu \mathrm{Pa})$ of the transmitted signal at time $t$ (second). Function $r(t)$ represents the amplitude $(\mu \mathrm{Pa})$ of the received signal as a function of time $t$. Attenuation, delays and ambient noise are considered in the model. The transmitter and receiver are assumed to be stationary. The emphasis of this work is on the model of the channel simulator. The modulator and demodulator are the work of Borowski [8].

The channel simulator consists of a fading model $c(t)$ and an ambient noise model $n(t)(\mu \mathrm{Pa})$ [37], see Figure 4.2. Multipath fading is calculated and applied. Then, ambient noise is added. The channel simulator is modeled as the following equation:

$$
r(t)=s(t) * c(t)+n(t)
$$

Function $n(t)$ is the ambient noise at time $t$. The operator ${ }^{(*)}$ means convolution. Function $c(t)$ is the impulse response of the fading model as a function of time $t$,

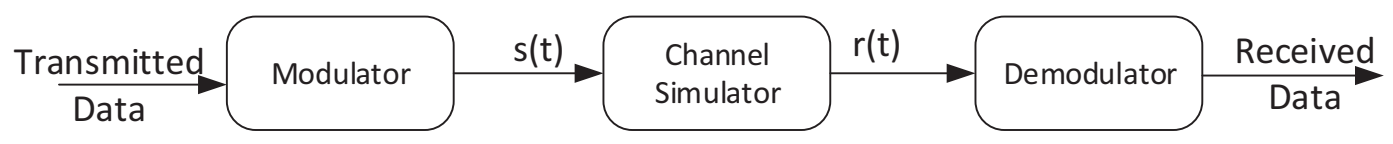

Figure 4.1: Underwater acoustic communication model. 


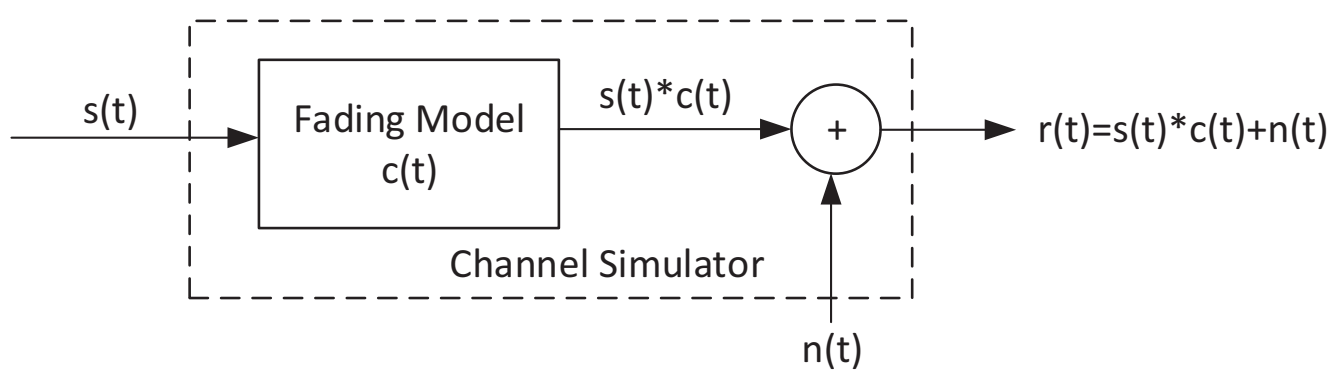

Figure 4.2: Underwater channel simulator.

represented by the following equation:

$$
c(t)=\sum_{k=1}^{L} a_{k} \delta\left(t-\tau_{k}\right)
$$

Constant $L$ is the number of multipath components. Variable $a_{k}$ represents the attenuation factor of the $k$-th component. Variable $\tau_{k}$ is the time delay of the $k$-th component. The Dirac delta function $\delta(t)$ is the unit impulse [40]. It produces an impulse value 1 at time 0 and goes to 0 at any other time:

$$
\delta(t)=\left\{\begin{array}{l}
1, t=0 \\
0, t \neq 0
\end{array}\right.
$$

The function $\delta\left(t-\tau_{k}\right)$ is the unit impulse function at time $t-\tau_{k}$. In Equation 4.1, the convolution is defined as follow:

$$
s(t) * c(t)=\int_{-\infty}^{\infty} c(\tau) s(t-\tau) d \tau .
$$

In Equation 4.2, replacing $t$ with $\tau$, we get

$$
c(\tau)=\sum_{k=1}^{L} a_{k} \delta\left(\tau-\tau_{k}\right) .
$$

The integral in Equation 4.3 can be transformed to

$$
\int_{-\infty}^{\infty} \sum_{k=1}^{L} a_{k} \delta\left(\tau-\tau_{k}\right) s(t-\tau) d \tau
$$


Because the Dirac delta function $\delta\left(\tau-\tau_{k}\right)$ equals one only when $\tau$ is equal to $\tau_{k}$, the output of the fading model $s_{f a d}(t)$ is inferred as:

$$
s_{f a d}(t)=\sum_{k=1}^{L} a_{k} s\left(t-\tau_{k}\right)
$$

We employ different algorithms to compute the fading model. This chapter describes two time domain fading models. Section 4.1 introduces a baseline model. Section 4.2 presents a multipath-pressure-sum model in the time domain.

\subsection{Baseline}

This model is well documented in a book authored by Proakis [37]. We call it the baseline model. A transmitted modulated signal can be represented by a low-pass equivalent signal when it is bandpass filtered [37]. We assume that the signals are bandpass filtered. Therefore, we can apply the low-pass equivalent analysis. According to Proakis and Salehi [37], the low-pass equivalent model of a signal is represented as:

$$
s(t)=\operatorname{Re}\left(s_{l}(t) e^{j 2 \pi f_{c} t}\right)
$$

The operator Re extracts the real part of a complex signal. Function $s_{l}(t)$ is the low-pass equivalent of the transmitted signal as a function of time $t$. Variable $f_{c}$ is the signal center frequency. After the equivalent low-pass signal goes through the fading model of Equation 4.2, we get the response $s_{f a d}$ of the fading channel:

$$
s_{f a d}(t)=\operatorname{Re}\left(\sum_{k=1}^{L} a_{k} s_{l}\left(t-\tau_{k}\right) e^{j 2 \pi f_{c}\left(t-\tau_{k}\right)}\right)
$$

It is equivalent to the following equation:

$$
s_{f a d}(t)=\operatorname{Re}\left(\left[\sum_{k=1}^{L} a_{k} s_{l}\left(t-\tau_{k}\right) e^{-j 2 \pi f_{c} \tau_{k}}\right] e^{j 2 \pi f_{c} t}\right)
$$

Using Equation 4.5, we get the received low-pass equivalent signal $r_{l}(t)$ without noise:

$$
r_{l}(t)=\sum_{k=1}^{L} a_{k} s_{l}\left(t-\tau_{k}\right) e^{-j 2 \pi f_{c} \tau_{k}} .
$$


Adding ambient noise $n(t)$, the low-pass equivalent received signal corresponds to the following equation:

$$
r(t)=\sum_{k=1}^{L} a_{k} s_{l}\left(t-\tau_{k}\right) e^{-j 2 \pi f_{c} \tau_{k}}+n(t)
$$

Function $r(t)$ is the received signal as a function of time $t$. Constant $L$ is the number of multipath components. Function $s_{l}\left(t-\tau_{k}\right)$ is the transmitted low-pass equivalent signal at time $t-\tau_{k}$. Variable $f_{c}$ is the center frequency of the signal. Variable $a_{k}$ is the attenuation factor of the $k$-th component. Variable $\tau_{k}$ is the delay of the $k$-th component. Function $n(t)$ is the noise at time $t$.

For every multipath component, the attenuation and delay are extracted from the arrival file (ending with the arr extension) produced by the BELLHOP software. For a given distance and a center frequency, only one BELLHOP simulation is needed. We do the calculation of the attenuation and delay for the center frequency only. It is running very quickly. We make abstraction of frequency dependent differences. Therefore, it results into a certain inaccuracy (see Chapter 7).

The running time of the algorithm can be approximately quantified by a time complexity [44]. The big $\mathcal{O}$ notation is used to express the time complexity. In the baseline model, there are two stages: one calculates the attenuation factors $\left(a_{k}\right)$ and delays $\left(\tau_{k}\right)$; the other adds attenuated signals together along all propagation paths. The attenuation factors and delays are obtained using a single invocation of the BELLHOP software. We abstract that invocation as a single operation. Therefore, the time complexity of the calculation of the attenuation factors and delays is of $\mathcal{O}(1)$. Let $L$ be the number of propagation paths, the running time of adding signals is $\mathcal{O}(L)$. The total time is $\mathcal{O}(1)+\mathcal{O}(L)$. Hence, the time complexity of this model is $\mathcal{O}(L)$.

\subsection{Multipath-Pressure-Sum}

The difference between the baseline model and this model is the calculation and application of the attenuation to every frequency component. The aim is to get a more accurate results. Indeed, attenuation is sensitive to frequency. The model of Equation 4.8 is transformed to the following equation: 


$$
r(t)=\sum_{f \in F} \sum_{k=1}^{L} a_{f, k} s_{l}\left(t-\tau_{k}\right) e^{-j 2 \pi f \tau_{k}}+n(t)
$$

Function $r(t)$ is the channel output signal as function of time $t$. Constant $L$ is the number of multipath components. Variable $a_{f, k}$ is the attenuation factor at frequency $f$ of the $k$-th component. Variable $\tau_{k}$ is the delay of the $k$-th component. Function $s_{l}\left(t-\tau_{k}\right) e^{-j 2 \pi f \tau_{k}}$ is the lowpass equivalent input signal (Equation 4.5). Function $n(t)$ is the noise at time $t$. Variable $f$ is a frequency component. Variable $F$ corresponds to the bins of a Fast Fourier Transform (FFT). Let $f_{\min }$ and $f_{\max }$ be the minimum and maximum frequencies of the signal, respectively. Variable $N$ is the number of frequencies. Variable $F$ can be calculated as:

$$
F=\left\{f_{\min }+\frac{f_{\max }-f_{\min }}{N} \cdot i: i=0,1, \ldots, N-1\right\}
$$

The determination of the number of frequency components $N$ depends on the sampling frequency, number of bits per data packet and bit rate. According to the Nyquist sampling theorem, the sampling frequency has to be greater than twice the maximum frequency. However, it is not practical for a bandpass signal due to the required large sampling frequency. Harmonic sampling, or bandpass sampling, works better for a bandpass signal [5]. With harmonic sampling, the sampling frequency $f_{s}$ has to be greater than twice the difference $f_{\max }-f_{\min }$, i.e, the signal bandwidth:

$$
f_{s}>2\left(f_{\max }-f_{\min }\right) \text { samples/second. }
$$

Given a buffer of size, in bits, and a signal data rate bit_rate, in bits per second, the number of buffered samples is:

$$
n=\frac{\text { size } \cdot f_{s}}{\text { bit_rate }} \text { samples. }
$$

Overall, the number of signal components is determined by the following equation:

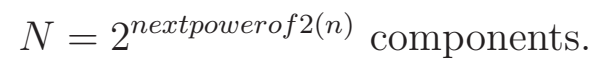

The operation nextpowerof $2(n)$ returns the smallest power of two that is equal to or larger than the absolute value of $n$. 
This model includes calculating the attenuation factors $\left(a_{k}\right)$ and delays $\left(\tau_{k}\right)$, at each frequency, and adding attenuated pressures of received signals together along all propagation paths for all frequencies. The attenuation factors and delays are obtained using the BELLHOP software for each frequency. Let $N$ be the number of frequency components, the time complexity of the calculation of attenuation factors and delays is $\mathcal{O}(N)$. Let $L$ be the number of propagation paths, the time complexity of adding received pressures is $\mathcal{O}(N L)$. The total time complexity is $\mathcal{O}(N)+O(N L)$, that is, $\mathcal{O}(N L)$. 


\section{Chapter 5}

\section{Frequency Domain Modeling}

Since underwater absorption of an acoustic signal is sensitive to frequency, we explore the relevance of modeling transmission loss in the frequency domain. The transmission loss is calculated for and applied to each frequency component of the signal.

Harmonic sampling is used because it can greatly reduce the sampling rate. The sampling frequency $f_{s}$ is calculated as Equation 4.11. The input analog signal goes through a Chebyshev Type 1 bandpass filter. The low cutoff frequency of the bandpass filter is set to $f_{\min }$ and high cutoff frequency is $f_{\max }$. To transform a signal from the time domain to the frequency domain, the Fast Fourier Transform (FFT) is used. Figure 5.1 shows the spectrum of an analog signal after going through a bandpass filter. Then, the analog signal is digitized by a modulator, shown as Figure 5.2.

The procedure for the calculation of the attenuation in the frequency domain consists of the following steps:

Step 1: For each frequency $f \in F$ and path index $k$, compute the attenuation $a_{f, k}$, with $a_{f, k}$ as in Equation 4.9 and $F$ as in Equation 4.10.

Step 2: For each frequency $f \in F$, use a FFT to get $y_{f}$, the amplitude of frequency component $f$.

Step 3: For each frequency $f \in F$ and path index $k$, get the amplitude of frequency component $f$ after attenuation.

$$
Y_{f}=a_{f, k} \cdot y_{f}
$$

Step 4: Transform the resulting signal to the time domain using an inverse FFT.

For Step 1, we explore the calculation of the attenuation with two groups of models: incoherent and coherent. The following sections introduce the models we use to compute incoherent and coherent attenuation. 


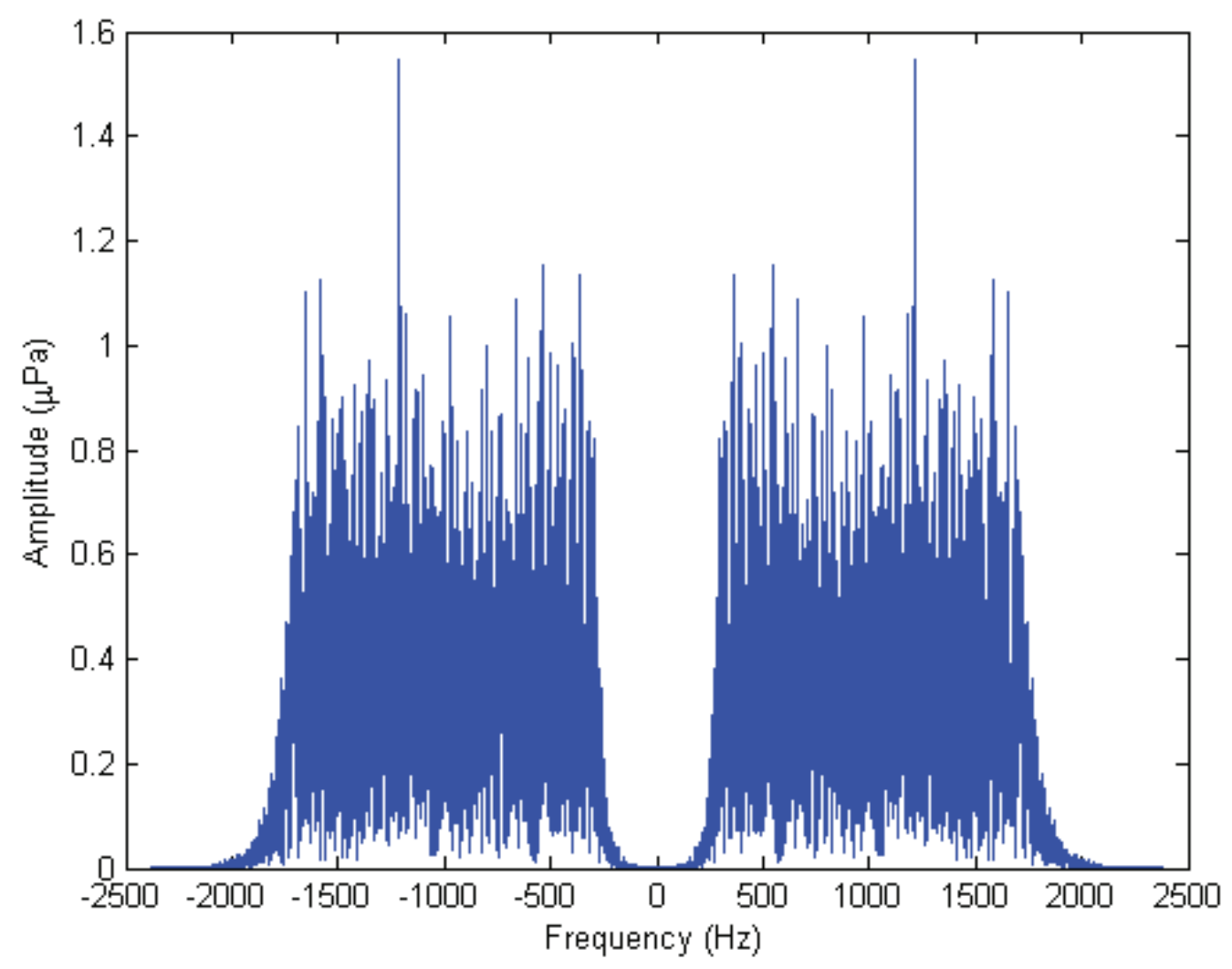

Figure 5.1: Spectrum of an analog signal with bandwidth 2 kilohertz after going through a bandpass filter. 


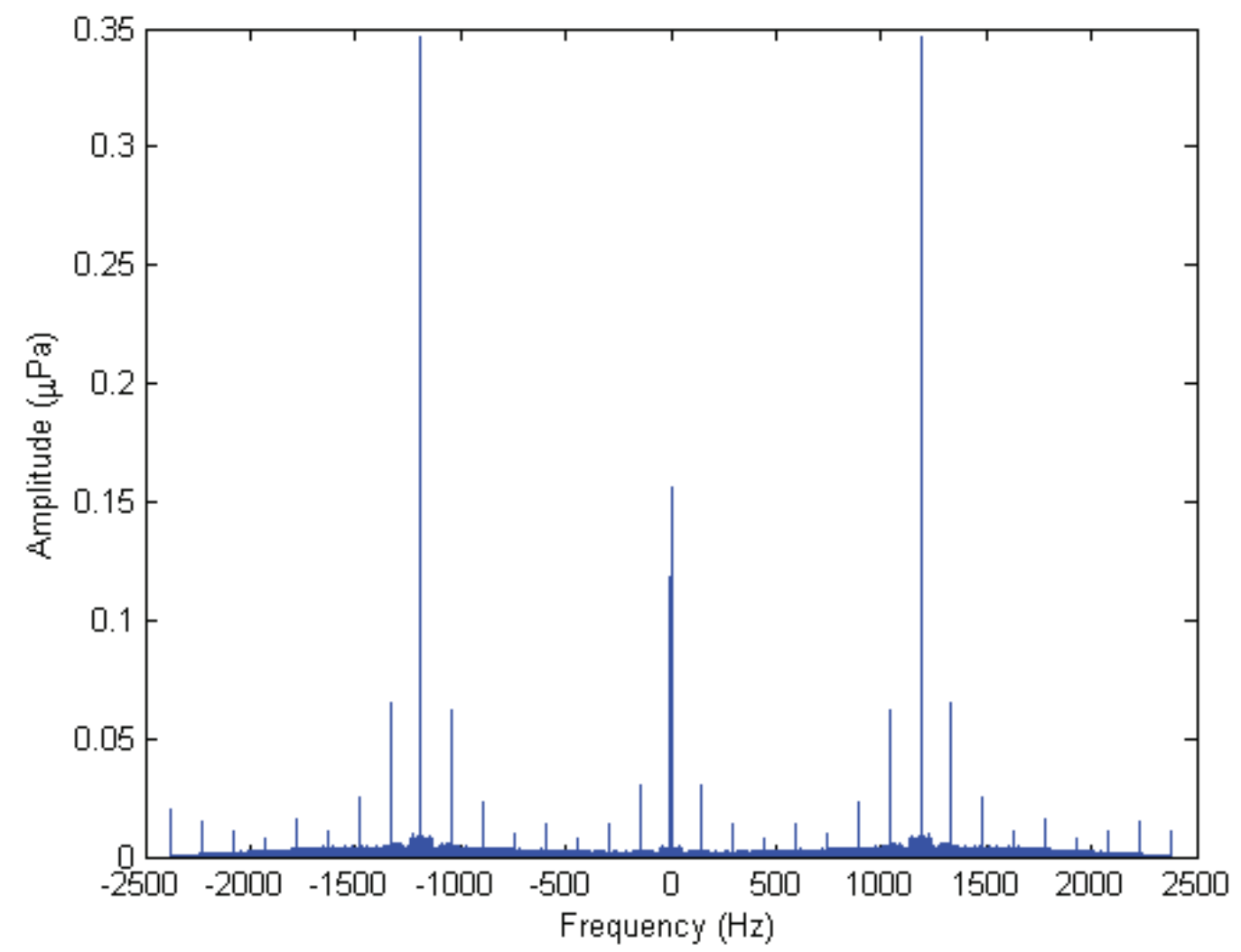

Figure 5.2: Spectrum of a signal after BPSK modulation. 


\subsection{Incoherent Attenuation}

This model is called incoherent because we make abstraction of phase differences between signals coming from different paths. We ignore the interference created by these phase differences. This model adds together the signals of all paths, without any phase effect.

In this section, we describe four models for incoherent attenuation calculation. Subsection 5.1.1 describes the multi-frequency-merge-path model. The incoherent acoustic pressure of all merged paths is calculated for each frequency component using the BELLHOP software. The acoustic pressure is turned into the attenuation. Subsection 5.1.2 introduces the multi-frequency-pressure-sum model. It uses the acoustic pressure for each eigenray using the BELLHOP software to calculate the received incoherent acoustic pressure. For every frequency, the sum of acoustic pressures of all eigenrays is turned into one attenuation value. Subsection 5.1.3 describes the multi-frequency-merge-path down conversion model. The spectrum of a broadband signal is down converted to baseband. Subsection 5.1.4 presents the linear fitting model to improve the efficiency of the multi-frequency-merge-path model.

\subsubsection{Multi-Frequency-Merge-Path}

In this model, we calculate the incoherent acoustic pressure for each frequency component $f \in F$, determined by Equation 4.10. The incoherent acoustic pressure calculation of the BELLHOP software is used, see Appendix B for details. In BELLHOP, each eigenray is traced. Accumulated acoustic pressure and phase along each eigenray are calculated [31], therefore, there is only one path index. The incoherent acoustic pressure calculation ignores phase differences. For an eigenray, the received acoustic pressure $p_{r}$ is the square root of the sum of the squared eigenray segment acoustic pressures, weighted by their corresponding Gaussian amplitude factor:

$$
p_{r}=\sqrt{\sum_{i} W_{i} A_{i}^{2}} \mu \mathrm{Pa}
$$

Variable $i$ is the index of an eigenray. Variable $W_{i}$ is the Gaussian amplitude factor

of eigenray $i$. It depends on the sound speed profile, propagation range and depth of 
transmitted and received signal [31]. Variable $A_{i}$ is the acoustic pressure of the $i$-th eigenray. The attenuation is the pressure loss, determined by the ratio of the received acoustic pressure $p_{r}$ and transmitted acoustic pressure $p_{t}$ :

$$
a_{f, 1}=\frac{p_{r}}{p_{t}}
$$

In the BELLHOP software, the transmitted acoustic pressure $p_{t}$ is $1 \mu \mathrm{Pa}$. Therefore, the attenuation equals to the received acoustic pressure.

In this model, there are three stages: the first transforms the signal from the time domain to the frequency domain using the FFT; the second calculates the received pressure for each frequency; the third converts the received pressure to attenuation for each frequency. Let $N$ be the number of frequency components, the time complexity of the FFT is $\mathcal{O}(N \log N)$ [29]. The attenuation factors are obtained using an invocation of the BELLHOP software for each frequency. For each frequency, BELLHOP outputs the attenuation factor for each path. Because of Equation 5.2, we view each invocation of the BELLHOP software as $L$ operations, where $L$ is the number of propagation paths. The time complexity of the calculation of the attenuation is $\mathcal{O}(N L)$. The time complexity of the third stage is $\mathcal{O}(N)$. Therefore, the total time complexity is $\mathcal{O}(N \log N)+\mathcal{O}(N L)+\mathcal{O}(N)$, that is, $\mathcal{O}(N L)$, with the reasonable assumption that $L$ is greater than or equal to $\log N$.

\subsubsection{Multi-Frequency-Pressure-Sum}

In this model, the BELLHOP software is used to calculate the incoherent acoustic pressure (relative to a source of $1 \mu \mathrm{Pa}$ ) for each frequency component and each path (corresponding to an eigenray). The Thorpe model is used to compute the attenuation using BELLHOP [41]. BELLHOP uses the following absorption equation:

$$
\alpha(f)=\frac{44 f^{2}}{4100+f^{2}}+\frac{0.11 f^{2}}{1+f^{2}}+3 \cdot 10^{-4} f^{2}+3.3 \cdot 10^{-3} \mathrm{~dB} / \mathrm{m}
$$

Variable $f$ is the signal frequency in KHz. The absorption is converted from $\mathrm{dB} / \mathrm{m}$ to nepers/m using the following equivalence:

$$
\alpha(f)=\frac{\alpha(f)}{8.69} \text { nepers } / \mathrm{m} \text {. }
$$


The received acoustic pressure $p_{f, k}$ on path $k$ is calculated by the following equation:

$$
p_{f, k}=p_{t} \cdot e^{-\alpha(f) r} \mu \mathrm{Pa}
$$

Constant $r$ (in meter) is the wave propagation distance between the source and destination on path $k$. For each $f \in F$, we sum the acoustic pressures of different paths:

$$
p_{r}=\sum_{k} p_{f, k} \mu \mathrm{Pa}
$$

We transform the acoustic pressure to attenuation using Equation 5.3.

In this model, there are four stages: the first transforms the signal from the time domain to the frequency domain using the FFT; the second calculates the received acoustic pressures along all paths for each frequency; the third adds received pressures along all paths for each frequency; the fourth converts the received pressures to attenuation factors for each frequency. Let $N$ be the number of frequency components, the time complexity of the FFT is $\mathcal{O}(N \log N)$. Let $L$ be the number of propagation paths, the time complexity of the calculation of the received pressures is $\mathcal{O}(N L)$. The time complexity of adding all pressures is $\mathcal{O}(N L)$. The time complexity for the fourth stage is $\mathcal{O}(N)$. The total time complexity is $\mathcal{O}(N \log N)+\mathcal{O}(N L)+\mathcal{O}(N L)+\mathcal{O}(N)$, that is, $\mathcal{O}(N L)$, with the reasonable assumption that $L$ is greater than or equal to $\log N$.

\subsubsection{Multi-Frequency-Merge-Path Down Conversion}

In this model, the spectrum of the signal is down converted to baseband. The purpose of this model is to speed up the FFT calculation. The number of frequency bins is reduced. The down conversion preserves the envelope of the spectrum. The properties of the spectrum are maintained. However, equivalent FFT analysis can be done with less samples and less frequency bins. The connection between the signal center frequency and bins is described by Equation 4.12.

For every component of a signal, the attenuation is calculated in broadband, i.e., before down conversion. We apply the obtained attenuation to the baseband signal, i.e., after down conversion. Let $f_{1}, \ldots, f_{i}, \ldots, f_{n}$ be the frequency components of the baseband signal, they are mapped to corresponding broadband frequency components 
$f_{w 1}, \ldots, f_{w i}, \ldots, f_{w n}$ using the following equation:

$$
f_{w i}=f_{i}+\left(f_{w c}-f_{c}\right) \mathrm{Hz}
$$

Constant $f_{w c}$ is the signal center frequency in broadband. Constant $f_{c}$ is the signal center frequency in baseband. The broadband attenuation is mapped to baseband attenuation, as follows:

$$
A_{f_{i}}=A_{f_{w i}} .
$$

The down conversion process causes a little bit of distortion, as demonstrated in the following experiment, see Figures 5.3, 5.4 and 5.5. Figure 5.3 shows the half spectrum of a digital data signal with center frequency 20 kilohertz and data rate 1 kbps. The distance is 200 kilometer. The spectrum is shown before and after attenuation. Before attenuation, the acoustic pressure peaks at almost up to $4.57 \cdot 10^{7}$ $\mu \mathrm{Pa}$. After attenuation, the signal at 20 kilohertz is very weak with respect to the noise between 19 kilohertz and 19.4 kilohertz (it peaks at almost up to $1.91 \cdot 10^{-74}$ $\mu \mathrm{Pa})$.

Figure 5.4 shows the digital data signal of Figure 5.3 down converted from 20 kilohertz to 1 kilohertz. We have half spectrum before and after attenuation. With attenuation, before down conversion, the peak amplitude is $4.57 \cdot 10^{7} \mu \mathrm{Pa}$. After down conversion, the peak amplitude is $2.82 \cdot 10^{-74} \mu \mathrm{Pa}$.

Figure 5.5 compares the BER obtained with no down conversion with the BER obtained with down conversion (there are 1000 trials). The BER obtained with no down conversion is better than the BER obtained with down conversion because the received signal has more energy and no distortion.

We estimate the error of down conversion with the Mean Square Error $(M S E)$ and Mean Absolute Percentage Error ( $M A P E)$ methods. The equation for the $M S E$ is:

$$
M S E=\frac{\sum_{i=1}^{n}\left(y_{i}-z_{i}\right)^{2}}{n}
$$

Constant $n$ is the number of trials. Variable $y_{i}$ is the BER of trial $i$ before down conversion $(i=1, \cdots, n)$. Variable $z_{i}$ is the BER of trial $i$ with down conversion. The equation for the $M A P E$ is:

$$
M A P E=\frac{1}{n} \sum_{i=1}^{n}\left|\frac{y_{i}-z_{i}}{y_{i}}\right|
$$



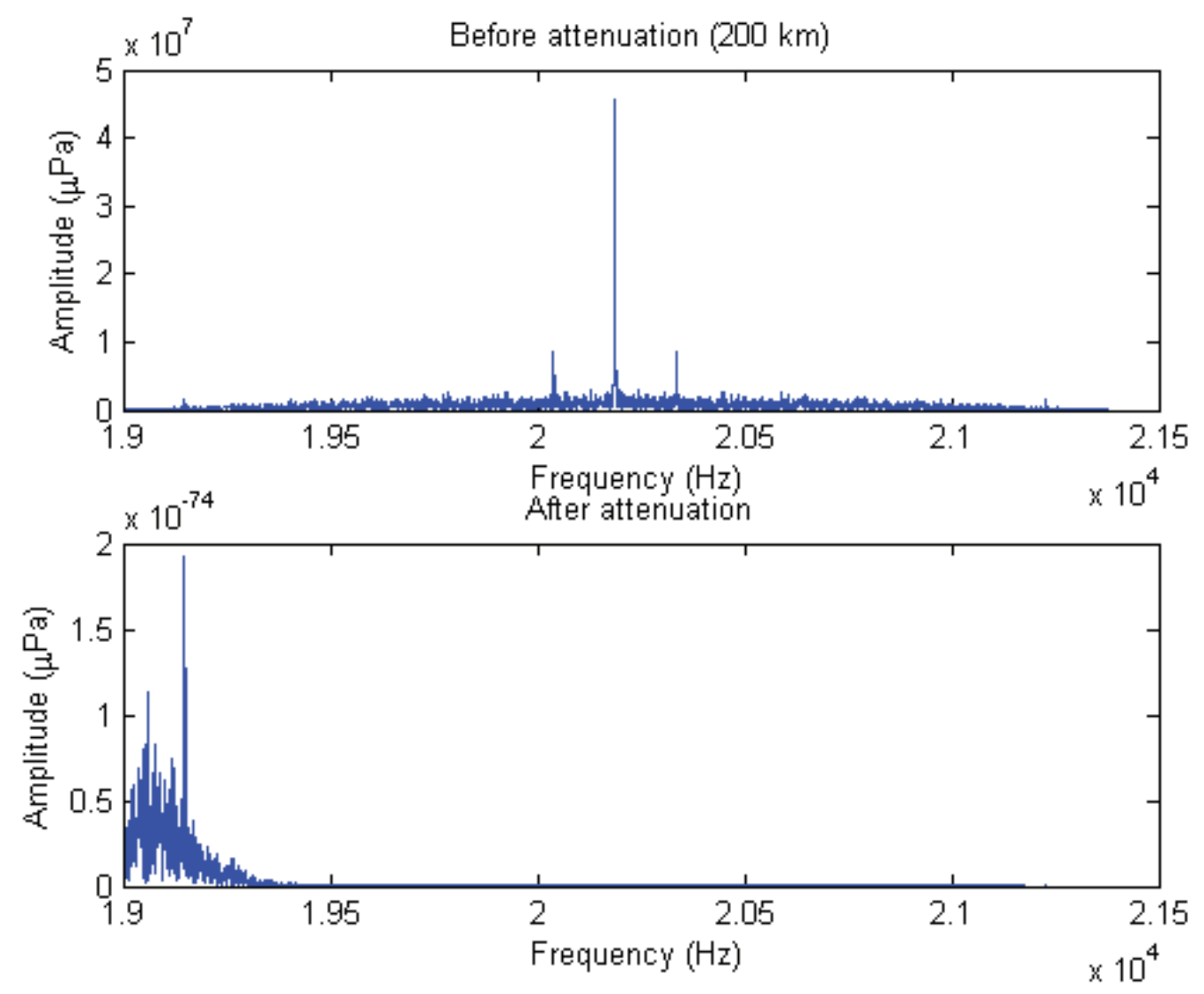

Figure 5.3: Half spectrum before and after attenuation ( $f_{c}$ is 20 kilohertz). 

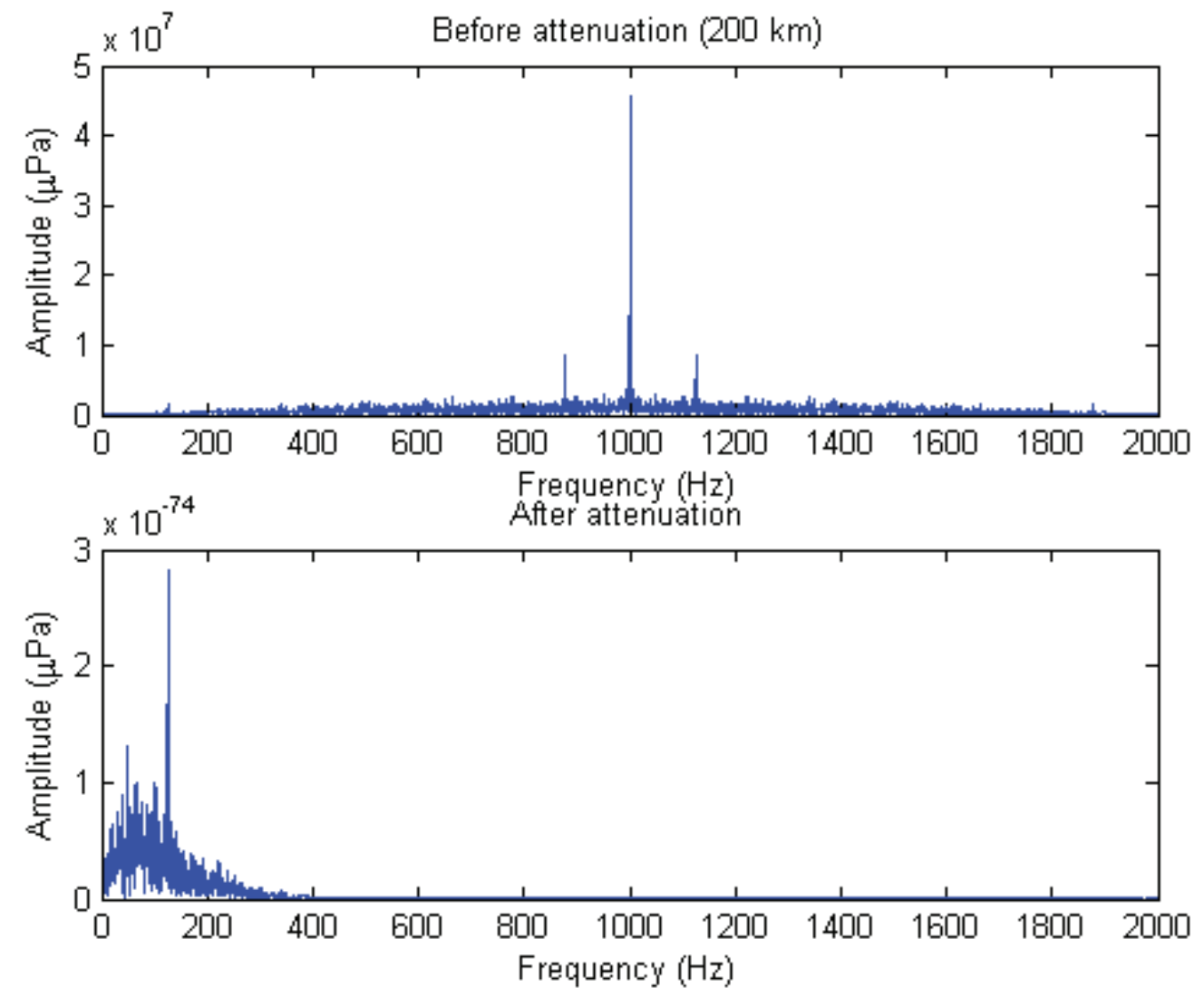

Figure 5.4: Half spectrum before and after attenuation with down conversion $\left(f_{c}\right.$ is 1 kilohertz). 


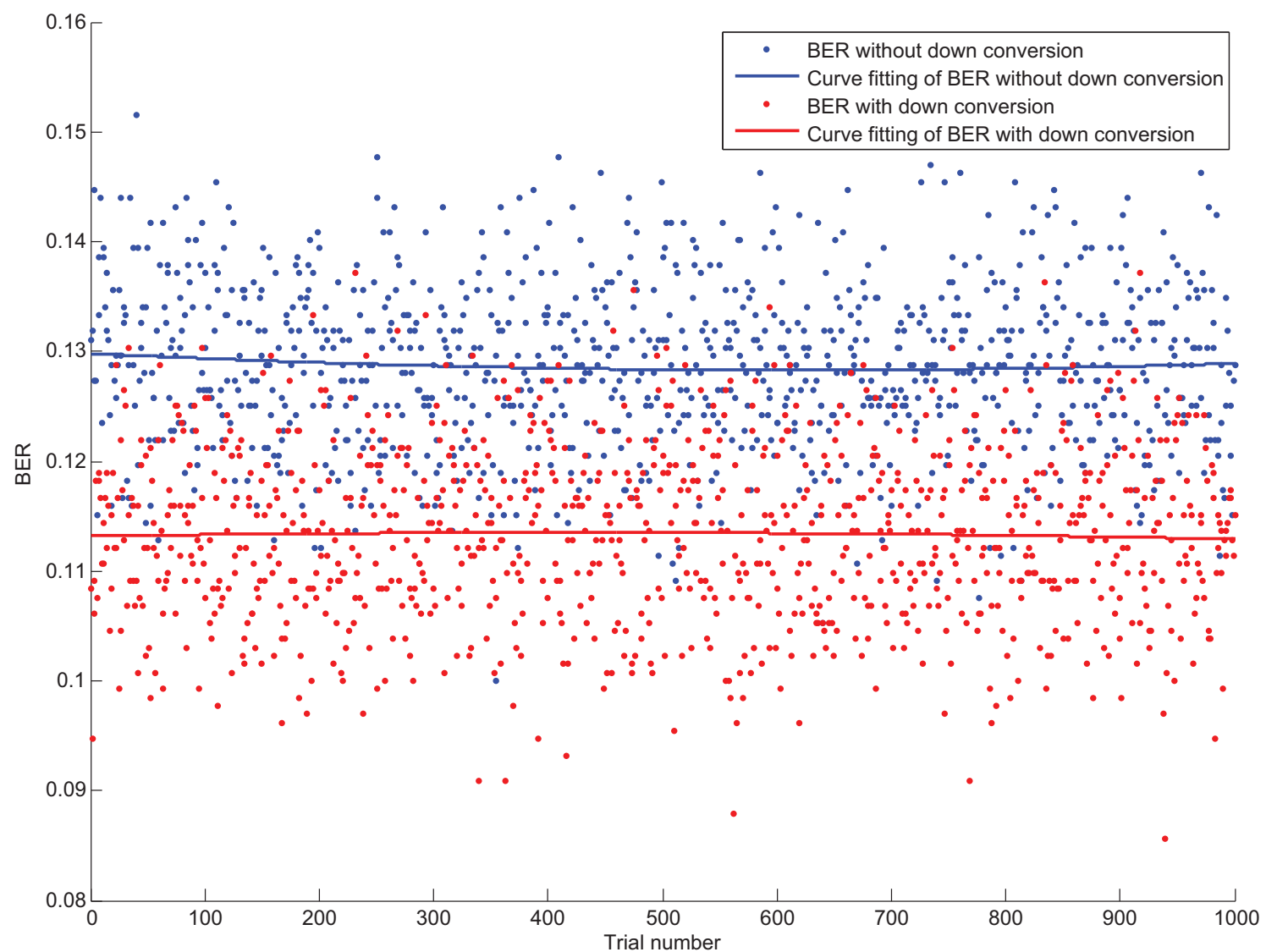

Figure 5.5: BER of the no down conversion - down conversion comparison. 
MSE shows a very tiny error, $0.024 \%$, between the BERs of the two methods. Whereas, MAPE presents a significant error with $11.9 \%$.

In this model, there are three stages: the first transforms the signal from the time domain to the frequency domain using the FFT; the second calculates the received pressure for each frequency; the third converts the received pressures to attenuation factors for each frequency. Let $N$ be the number of frequency components, the time complexity of the FFT is $\mathcal{O}(N \log N)$. The received pressures are obtained using an invocation of the BELLHOP software for each frequency. For each frequency, BELLHOP outputs the received pressure for each path. Because of Equation 5.2, we view each invocation of the BELLHOP software as $L$ operations, where $L$ is the number of propagation paths. Therefore, the time complexity of the calculation of the received pressures is $\mathcal{O}(N L)$. The time complexity for the third stage is $\mathcal{O}(N)$. Therefore, the total time complexity is $\mathcal{O}(N \log N)+O(N L)+O(N)$, that is, $\mathcal{O}(N L)$, with the reasonable assumption that $L$ is greater than or equal to $\log N$.

\subsubsection{Linear Fitting}

Using a three dimensional graph, Figure 5.6 shows the attenuation $(\mathrm{dB})$ as a function of frequency from 19 to 21 kilohertz and transmitter to receiver separation distance between 5 to 20 kilometer. Attenuation, in dB, is linear with respect to frequency. For a fixed distance (20 kilometer), Figure 5.7 shows the attenuation in $\mathrm{dB}$ as a function of frequency between 19 and 21 kilohertz. The environment is as Table A.1 in Appendix A. In blue, we have the incoherent attenuation obtained with the multi-frequencymerge-path model (see Section 5.1.1). In red, we have the incoherent attenuation obtained using linear fitting. Using the BELLHOP software, we calculate the attenuations at the lower-end frequency $\left(f_{l}\right)$ and upper-end frequency $\left(f_{u}\right)$ of the signal bandwidth. Variable $y_{l}$ is the acoustic pressure at the lower-end frequency. Variable $y_{u}$ is the acoustic pressure at the upper-end frequency. For any other frequency $f$, the attenuation is determined using the following equation:

$$
y=\left[\frac{y_{u}-y_{l}}{f_{u}-f_{l}}\right] \cdot\left(f-f_{l}\right)+y_{l}
$$

Although there are small fluctuations, attenuation is matched quite well by the line. Hence, attenuation can be represented quite well with the linear model. 


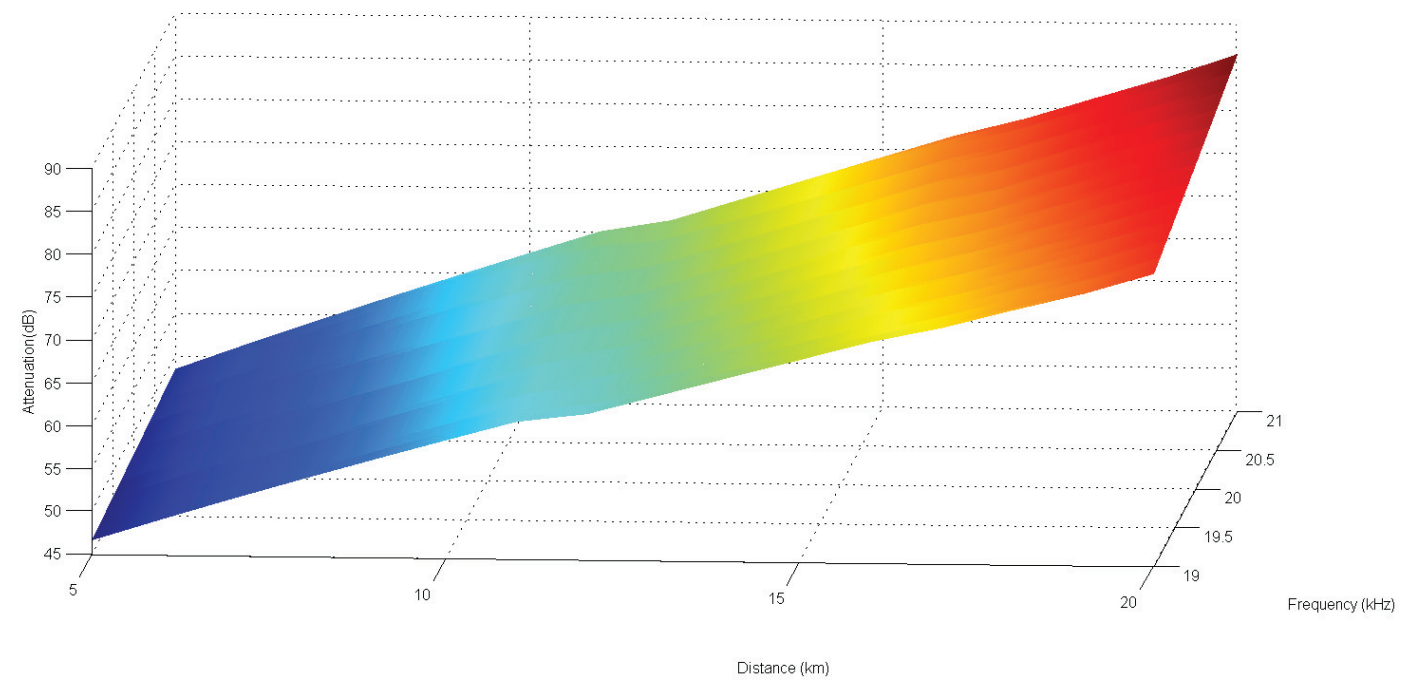

Figure 5.6: Attenuation as a function of frequency and distance.

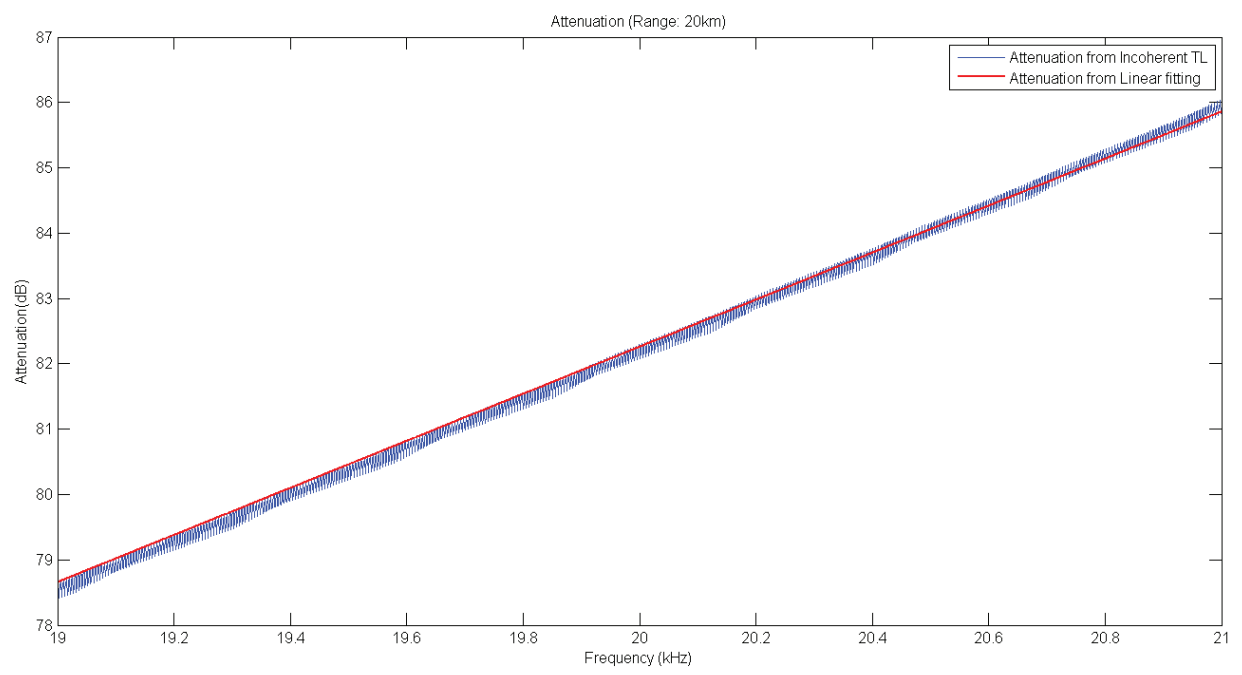

Figure 5.7: Attenuation of linear fitting. 
In this model, there are three stages: the first transforms the signal from the time domain to the frequency domain using the FFT; the second calculates the received pressure for each frequency; the third converts the received pressure to an attenuation factor for each frequency. Let $N$ be the number of frequency components, the time complexity of the FFT is $\mathcal{O}(N \log N)$. Let $L$ be the number of propagation paths, the time complexity of the calculation of the attenuation is $\mathcal{O}(L)$. The time complexity for the third stage is $\mathcal{O}(N)$. The total time complexity is $\mathcal{O}(N \log N)+O(L)+O(N)$, that is, $\mathcal{O}(N \log N)$.

\subsection{Coherent Attenuation}

Coherent attenuation takes into account the phase differences of signals propagating through different paths. Subsection 5.2.1 describes the coherent multi-frequencymerge-path model. A coherent acoustic pressure for all merged paths is calculated for each frequency component using the BELLHOP software. The acoustic pressure is turned into an attenuation factor. Subsection 5.2.2 introduces the coherent multifrequency-pressure-sum model. It uses the coherent acoustic pressure and delay for each eigenray, obtained using the BELLHOP software, to calculate the received coherent acoustic pressure. For every frequency, the sum of received acoustic pressures of all eigenrays is turned into a single attenuation factor.

\subsubsection{Coherent Multi-Frequency-Merge-Path}

The BELLHOP software can calculate the attenuation in a coherent manner. It calculates attenuation $\left(a_{f, k}\right)$ and phase differences as a function of frequency:

$$
p=\sum_{i} W_{i} A_{i} e^{-i\left(\omega t+\phi_{i}\right)}
$$

Variable $p$ represents the coherent acoustic pressure. Variable $i$ is the index of each segment along an eigenray path. Variable $W_{i}$ is the Gaussian amplitude factor in the $i$-th step. Variable $A_{i}$ is the acoustic pressure in the $i$-th step. The term $\omega t+\phi_{i}$ is the phase at time $t$ in the $i$-th step where $\omega$, the angular frequency, is equal to $2 \pi f$. All paths are merged together in BELLHOP. In Equation 5.1, $k$ equals to 1. For multipath within a specific range, the coherent acoustic pressure can be acquired 
from the BELLHOP generated shade file (ending with the shd extension). The programming details can be found in Appendix B.

In this model, there are three stages: the first transforms the signal from the time domain to the frequency domain using the FFT; the second calculates the received pressure for each frequency; the third converts the received pressure to an attenuation factor for each frequency. Let $N$ be the number of frequency components, the time complexity of the FFT is $\mathcal{O}(N \log N)$. Let $L$ be the number of propagation paths, the time complexity of the calculation of the attenuation factors is $\mathcal{O}(N L)$. The time complexity for the third stage is $\mathcal{O}(N)$. The total time complexity is $\mathcal{O}(N \log N)+$ $O(N L)+O(N)$, that is $\mathcal{O}(N L)$, with the reasonable assumption that $L$ is greater than or equal to $\log N$.

\subsubsection{Coherent Multi-Frequency-Pressure-Sum}

The BELLHOP software is used to calculate attenuation and phase differences as a function of frequency and path. Let function $y_{f}$ be the transmitted signal strength at frequency $f$. Because of the linearity property of the FFT [37], we have that at frequency $f$ and on path $k$, the time domain representation of the received $a_{k} s(t)$ can be transformed to the frequency domain representation $a_{k} y_{f}$. Because of the time-shift property of the FFT [37], we have that at frequency $f$, the time domain representation of the received $s(t-\tau)$ can be transformed to the frequency domain representation $y_{f} e^{-j 2 \pi \tau}$.

Referring to Equation 4.4, the time domain model can be transformed to a frequency domain model with $\tau_{k}$ replaced by $\tau_{k}-\tau_{0}$, shown in the following equation:

$$
Y_{f}=\sum_{k=1}^{L} a_{k} y_{f} e^{-2 j \pi f\left(\tau_{k}-\tau_{0}\right)} .
$$

Function $Y_{f}$ is the received signal strength at frequency $f$. Constant $L$ is the total number of paths. Variable $a_{k}$ is the attenuation on the $k$-th path calculated by BELLHOP. Variable $\tau_{0}$ is the minimum delay among all eigenrays. Variable $\tau_{k}$ is the delay on the $k$-th path. Equation 5.11 is equivalent to the following equation:

$$
Y_{f}=y_{f} \sum_{k=1}^{L} a_{k} e^{-2 j \pi f\left(\tau_{k}-\tau_{0}\right)} .
$$


In this model, there are four stages: the first transforms the signal from the time domain to the frequency domain using the FFT; the second calculates the received acoustic pressures along all paths for each frequency; the third adds received pressures along all paths for each frequency; the fourth converts the received pressure to an attenuation factor for each frequency. Let $N$ be the number of frequency components, the time complexity of the FFT is $\mathcal{O}(N \log N)$. Let $L$ be the number of propagation paths, the time complexity of the calculation of the received pressures is $\mathcal{O}(N L)$. The time complexity of adding all received pressures is $\mathcal{O}(N L)$. The time complexity for the fourth stage is $\mathcal{O}(N)$. Therefore, the total time complexity is $\mathcal{O}(N \log N)+$ $O(N L)+O(N L)+O(N)$, that is, $\mathcal{O}(N L)$, with the reasonable assumption that $L$ is greater than or equal to $\log N$. Table 5.1 lists the time complexities of all the models.

Table 5.1: Time complexities of all models.

\begin{tabular}{|l|l|l|}
\hline Domain & Method & Time Complexity \\
\hline Time Domain & Baseline & $\mathcal{O}(L)$ \\
\hline Time Domain & Multipath-Pressure-Sum & $\mathcal{O}(N L)$ \\
\hline Frequency Domain & Multi-Frequency-Merge-Path & $\mathcal{O}(N L)$ \\
\hline Frequency Domain & Multi-Frequency-Pressure-Sum & $\mathcal{O}(N L)$ \\
\hline Frequency Domain & Multi-Frequency-Merge-Path Down Conversion & $\mathcal{O}(N L)$ \\
\hline Frequency Domain & Linear Fitting & $\mathcal{O}(N \log N)$ \\
\hline Frequency Domain & Coherent Multi-Frequency-Merge-Path & $\mathcal{O}(N L)$ \\
\hline Frequency Domain & Multi-Frequency-Pressure-Sum & $\mathcal{O}(N L)$ \\
\hline
\end{tabular}




\section{Chapter 6}

\section{Noise Modeling and Simulation}

The sources of noise in the ocean are classified as ambient or localized [50]. Ambient noise is caused by shrimps, fishes, and various mammals, which always exist in the background of the sea. Localized noise is only present in certain areas. In this project, we focus on ambient noise. White noise and colored noise are the two forms of ambient noise that we simulate.

White noise is defined as a random signal with a fixed energy density with respect to frequency [32]. Additive White Gaussian Noise (AWGN) is the simplest and most commonly used type of noise used in simulation. AWGN has two characteristics: additive and Gaussian. Additive means it can be added to other kinds of noise already present in the environment. Gaussian refers to the probability distribution of the density of the noise. Normally, the mean voltage of AWGN is zero and standard deviation is one. In contrast to AWGN, the density of colored noise is frequency dependent. In the underwater environment, the ambient noise is colored.

To evaluate the effect of noise in a communication channel, a common criteria is the Bit Error Rate (BER) versus the ratio $E_{b} / N_{0}$. The term $E_{b} / N_{0}$ is called the energy per bit to noise power spectral density or signal to noise ratio (SNR) per bit, which represents the energy per bit (Joule). $N_{0}$ is the noise spectral density (watts/Hz). $N_{0}$ is equal to the ratio $N / B$, where $N$ is the noise power and $B$ is the bandwidth.

In this project, white noise is generated according to a specific $E_{b} / N_{0}$ ratio. Let $N$ be the power of the noise (milliwatt), $P$ the power of the signal (milliwatt), $B$ the bandwidth (Hz) and $R$ the symbol rate (baud). We have the following equality:

$$
N=\frac{P \cdot B}{R \cdot\left(E_{b} / N_{0}\right)} \text { milliwatt }
$$

For $P$, we use the Root Mean Square $(R M S)$ power of the signal. Let $x_{1}, x_{2}, \ldots, x_{n}$ 
be $n$ discrete samples of a signal, we have:

$$
R M S=\sqrt{\frac{1}{n} \sum_{i=1}^{n} x_{i}^{2}} \text { milliwatt }
$$

In this project, to simulate white noise we use the MATLAB function awgn(). The generation of colored noise includes two steps: AWGN generation and low-pass filtering. The low-pass filter has a profile similar to the profile of the underwater colored ambient noise. More details can be found in Reference [6]. 


\section{Chapter 7}

\section{Simulation Results}

This chapter presents the results obtained with the simulation of all underwater communication fading models discussed in Chapters 4 and 5 along with the noise models introduced in Chapter 6. For each fading model, both white noise and colored noise models are implemented. BER versus $E_{b} / N_{0}$ curves obtained using different models are compared with the curve of a theoretical reference and baseline model.

Since the underwater environment is very complex, there is no universal underwater acoustic communication channel model [36]. In different environments, the same channel model produces different results. In our simulations, we use one environment, i.e., an environment with an isospeed sound profile and shallow water. See Appendix A for details.

All the simulations are conducted using the MATLAB and BELLHOP software. The main functions are written in MATLAB. BELLHOP is invoked by MATLAB functions to calculate the received acoustic pressures and delays. MATLAB obtains the values from BELLHOP by reading its output files. The BELLHOP interfaces and invoked methods are reviewed in Appendix B. Each model is simulated with a $95 \%$ statistical level of confidence. A target $E_{b} / N_{0}$ ratio, in the range from $2 \mathrm{~dB}$ to $12 \mathrm{~dB}$ [37], is used to determine the noise power. The obtained BER values are fitted to a curve using the MATLAB Gaussian model of curve fitting.

Section 7.1 presents the simulation results and analyses of the time domain models

explained in Chapter 4. Section 7.2 discusses the simulation results and analyses of the frequency domain models explained in Chapter 5. All our models are compared with a theoretical reference and baseline model. 


\subsection{Time Domain Simulation Results}

In this section, two time domain models are simulated and compared. Subsection 7.1.1 presents the BER vs. $E_{b} / N_{0}$ ratio curve obtained using the baseline model adding white noise or colored noise. The results of the simulation of the baseline model are compared with a theoretical BER curve. Subsection 7.1.2 presents the BER vs. $E_{b} / N_{0}$ ratio curve acquired using the multipath-pressure-sum model adding white noise or colored noise. The results of these simulations are compared with a theoretical BER curve and BER curve of the baseline model.

\subsubsection{Baseline}

Figure 7.1 shows three BER versus $E_{b} / N_{0}$ curves. The $x$-axis represents the $E_{b} / N_{0}$ ratio in $\mathrm{dB}$ form. The $y$-axis corresponds to the $\mathrm{BER}$ values. The blue dashed curve is the theoretical BER curve of BPSK modulation [46]. The red curve with asterisks is the BER curve derived from the simulation of the baseline model adding AWGN noise $(\mathrm{WN})$. The magenta curve with circles is the BER curve derived from the simulation of the baseline model adding colored noise $(\mathrm{CN})$.

The simulation results show that the BERs of the baseline model, with noise, are higher than the theoretical BERs. With colored noise, the BERs are larger than with AWGN noise. It is due to the frequency dependent feature of colored noise, which means that colored noise has more impact on the signal. When the $E_{b} / N_{0}$ ratio increases, i.e., the signal-to-noise ratio rises, the gap between the BER of the baseline $+W N$ curve and baseline $+C N$ curve are larger. The BERs with AWGN noise decrease more quickly than those with colored noise.

\subsubsection{Multipath-Pressure-Sum}

Figure 7.2 shows three BER versus $E_{b} / N_{0}$ curves. The $x$-axis represents the $E_{b} / N_{0}$ ratio. The $y$-axis corresponds to the BER values. The blue dashed curve is the theoretical BER curve of BPSK modulation [46]. The red curve with asterisks is the BER curve derived from the simulation of the multipath-pressure-sum model adding AWGN noise (WN). The magenta curve with circles is the BER curve derived from 


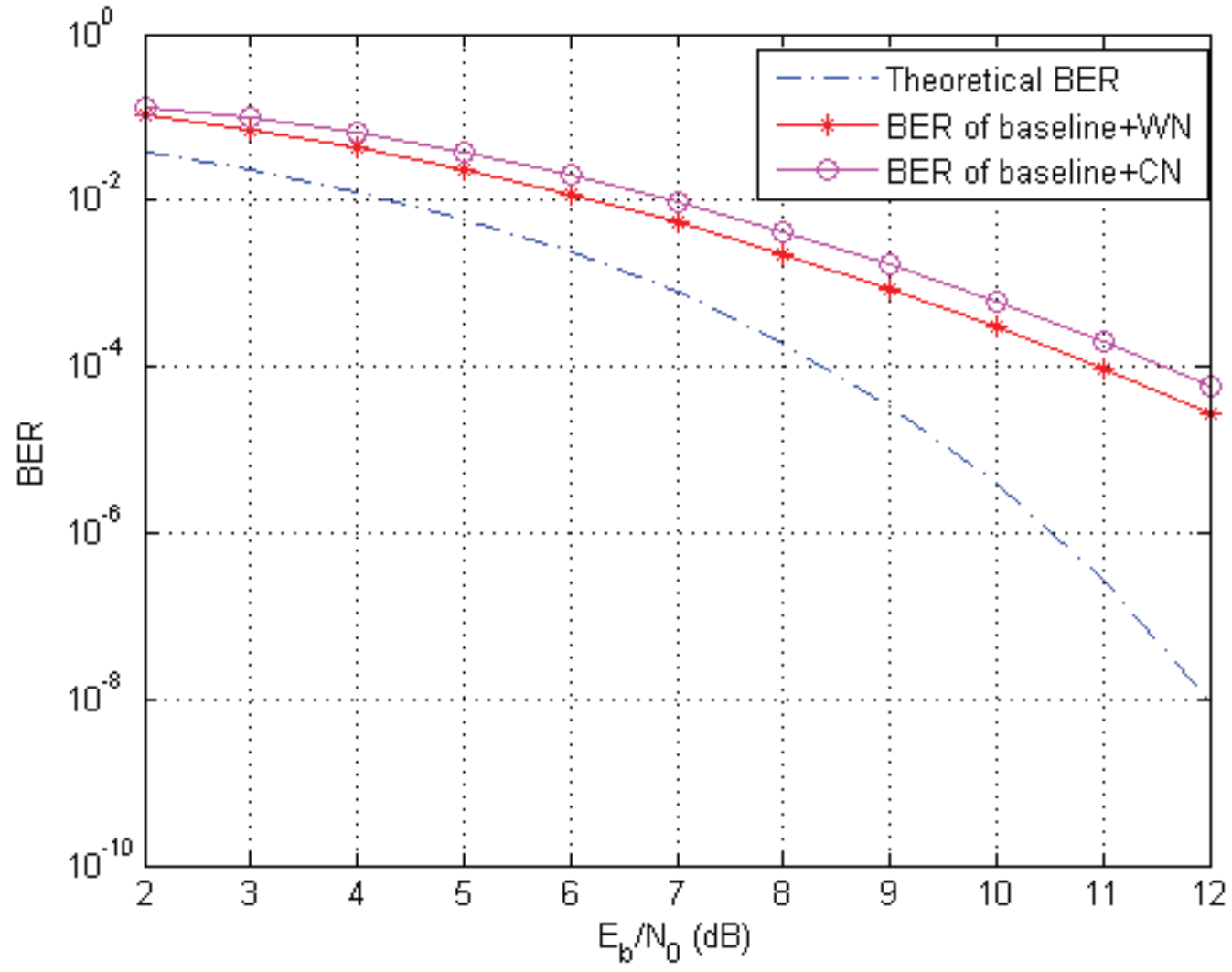

Figure 7.1: Theoretical BER of BPSK modulation vs. the BER of the baseline model. 


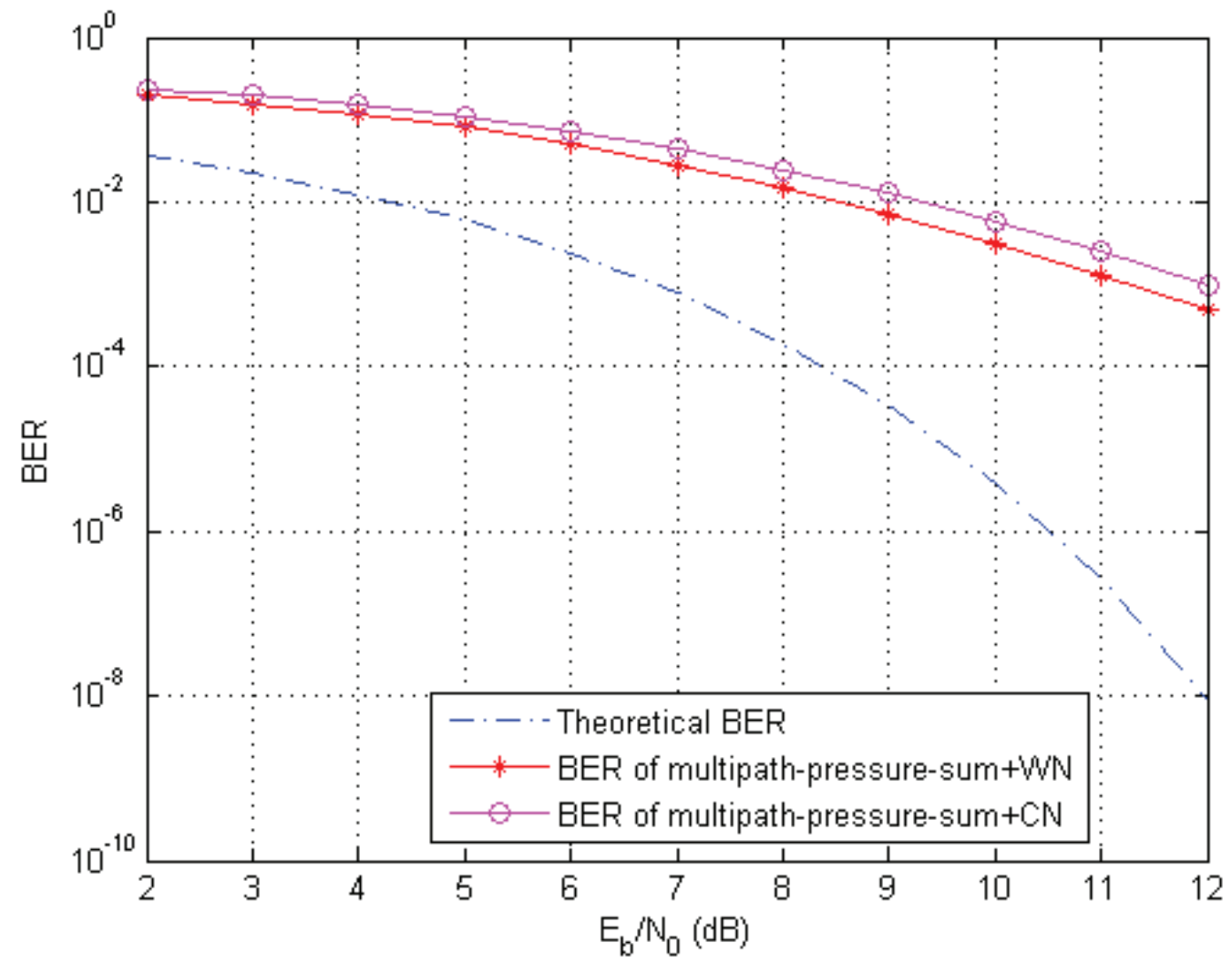

Figure 7.2: Theoretical BER of BPSK modulation vs. the BER of the multipathpressure-sum model.

the simulation of the multipath-pressure-sum model adding colored noise $(\mathrm{CN})$.

The simulation results show that the BERs of the multipath-pressure-sum model, with noise, are higher than the theoretical BERs. With colored noise, the BERs are larger than with AWGN noise. It is due to the frequency dependent feature of colored noise, which means that colored noise has more impact on the signal. When the $E_{b} / N_{0}$ ratio increases, i.e., the signal-to-noise ratio rises, the gap between the BER of the multipath-pressure-sum $+W N$ curve and multipath-pressure-sum $+C N$ curve are larger. The BERs with AWGN noise decrease more quickly than those with colored noise.

The BER curve of the multipath-pressure-sum is also compared with the one of the baseline model in Figure 7.3. The red curve with circles is the BER acquired from 


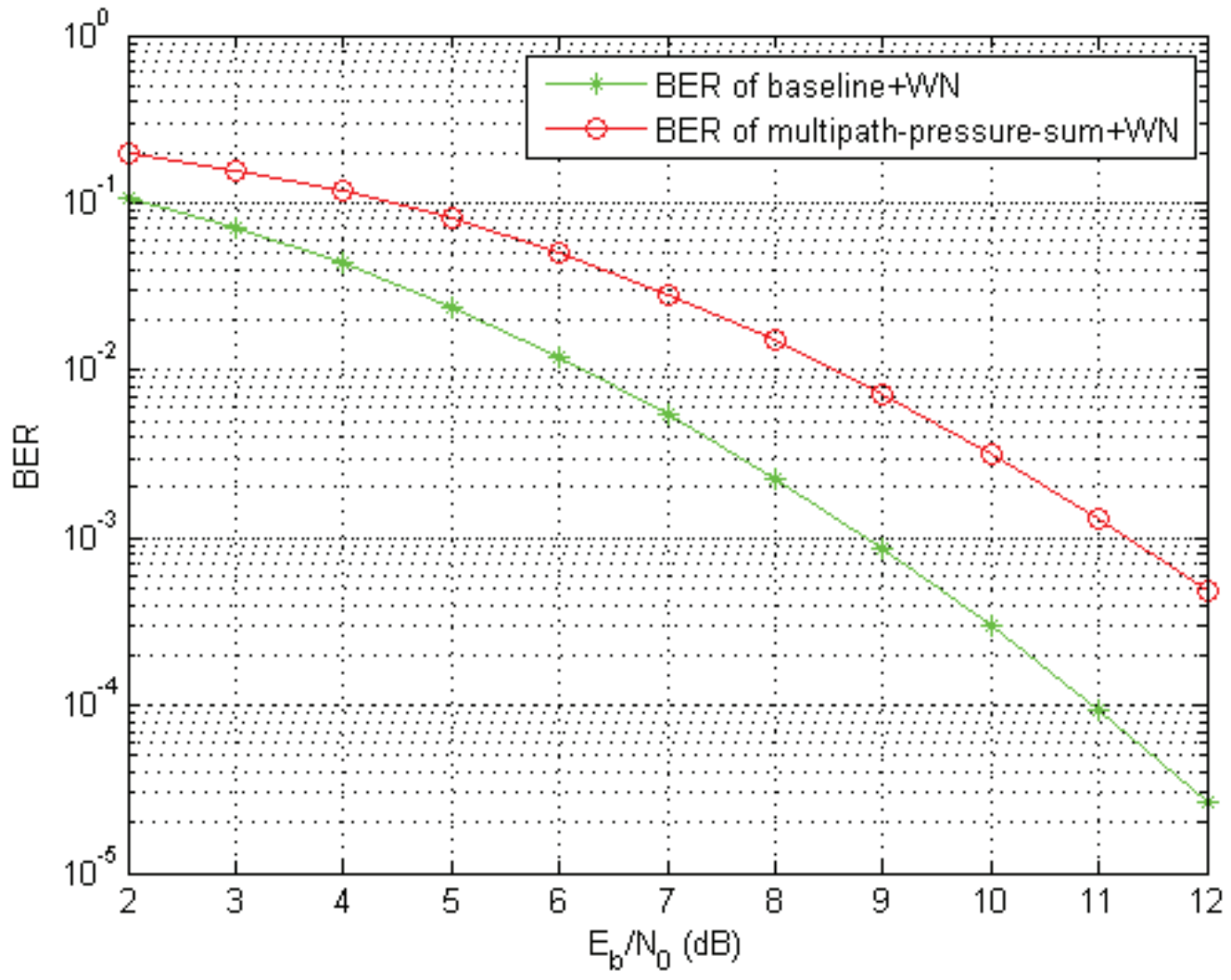

Figure 7.3: The BER of the baseline model vs. the BER of the multipath-pressuresum model.

the simulation of the multipath-pressure-sum model. The green one with asterisks the BER curve derived from the baseline model. Both of them are with AWGN noise. The BER values with the multipath-pressure-sum model are larger than the BER values with the baseline model. When the $E_{b} / N_{0}$ ratio becomes larger, the attenuation calculation increases the differences between the two models. 


\subsection{Frequency Domain Simulation Results}

In this section, two types of frequency domain models are simulated and compared. Subsection 7.2.1 presents the BER vs. $E_{b} / N_{0}$ ratio curve obtained using the incoherent models: multi-frequency-merge-path, multi-frequency-pressure-sum, multifrequency-pressure-sum down conversion and linear fitting, with white noise or colored noise. Subsection 7.2.2 presents the BER vs. $E_{b} / N_{0}$ ratio curve acquired using the incoherent models: coherent multi-frequency-merge-path and coherent multifrequency-pressure-sum, with white noise or colored noise. The results of all models are compared with a theoretical BER curve and BER curve of the baseline model.

\subsubsection{Incoherent Simulation Result}

\section{Multi-Frequency-Merge-Path}

Figure 7.4 shows three BER versus $E_{b} / N_{0}$ curves. The $x$-axis represents the $E_{b} / N_{0}$ ratio. The $y$-axis corresponds to the BER values. The blue dashed curve is the theoretical BER curve of BPSK modulation [46]. The red curve with asterisks is the BER curve derived from the simulation of the multi-frequency-merge-path model adding AWGN noise (WN). The magenta curve with circles is the BER curve derived from the simulation of the multi-frequency-merge-path model adding colored noise $(\mathrm{CN})$.

The simulation results show that the BERs of the multi-frequency-merge-path model, with noise, are higher than the theoretical BERs. With colored noise, the BERs are larger than with AWGN noise. It is due to the frequency dependent feature of colored noise, which means that colored noise has more impact on the signal. When the $E_{b} / N_{0}$ ratio increases, i.e., the signal-to-noise ratio rises, the gap between the BER of the multi-frequency-merge-path $+W N$ curve and multi-frequency-merge-path $+C N$ curve are larger. The BERs with AWGN noise decrease more quickly than those with colored noise.

The BER curve of the multi-frequency-merge-path is also compared with the one of the baseline model in Figure 7.5. The red curve with circles is the BER curve acquired from the simulation of the multi-frequency-merge-path model. The green 


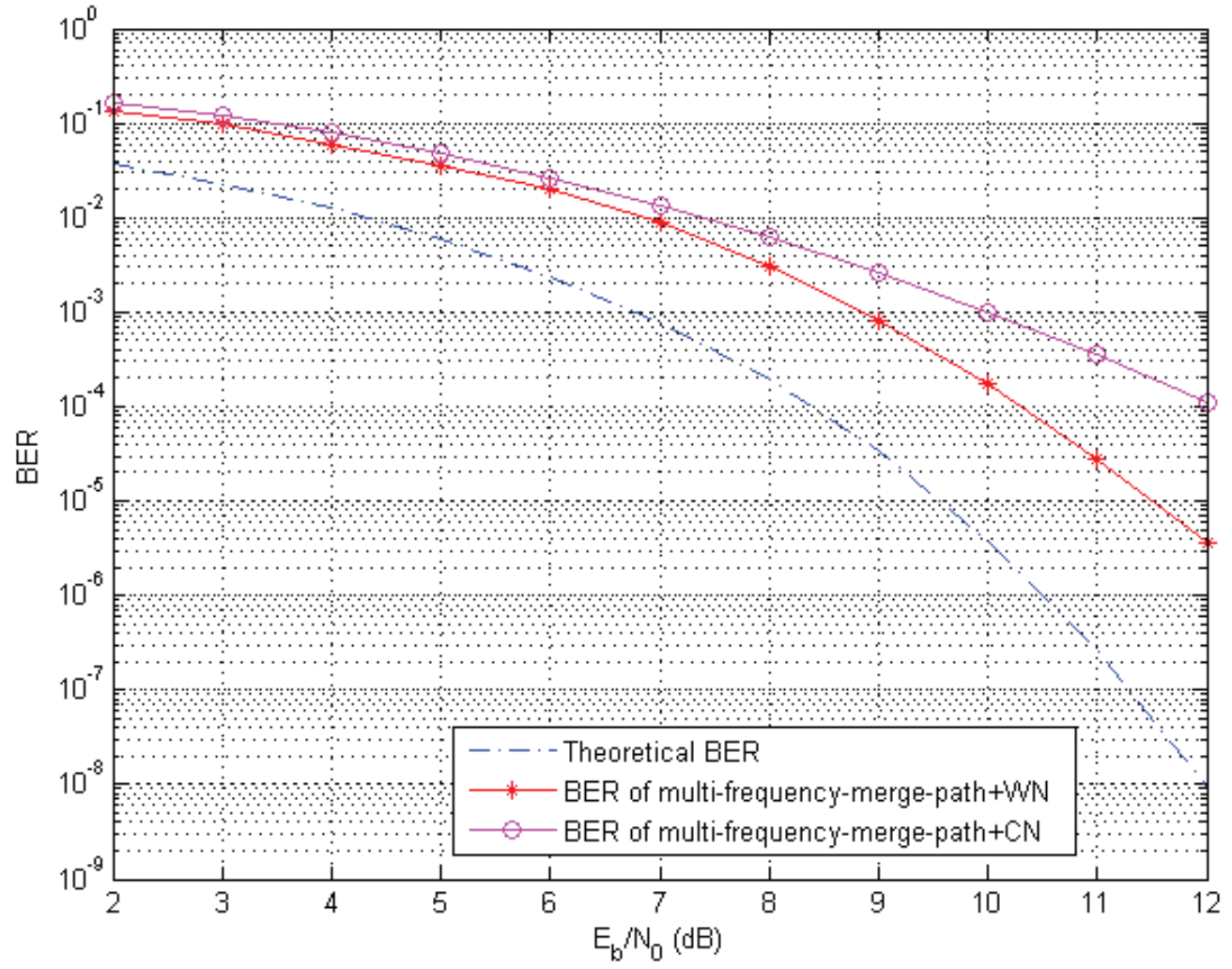

Figure 7.4: Theoretical BER of BPSK modulation vs. the BER of the multifrequency-merge-path model. 


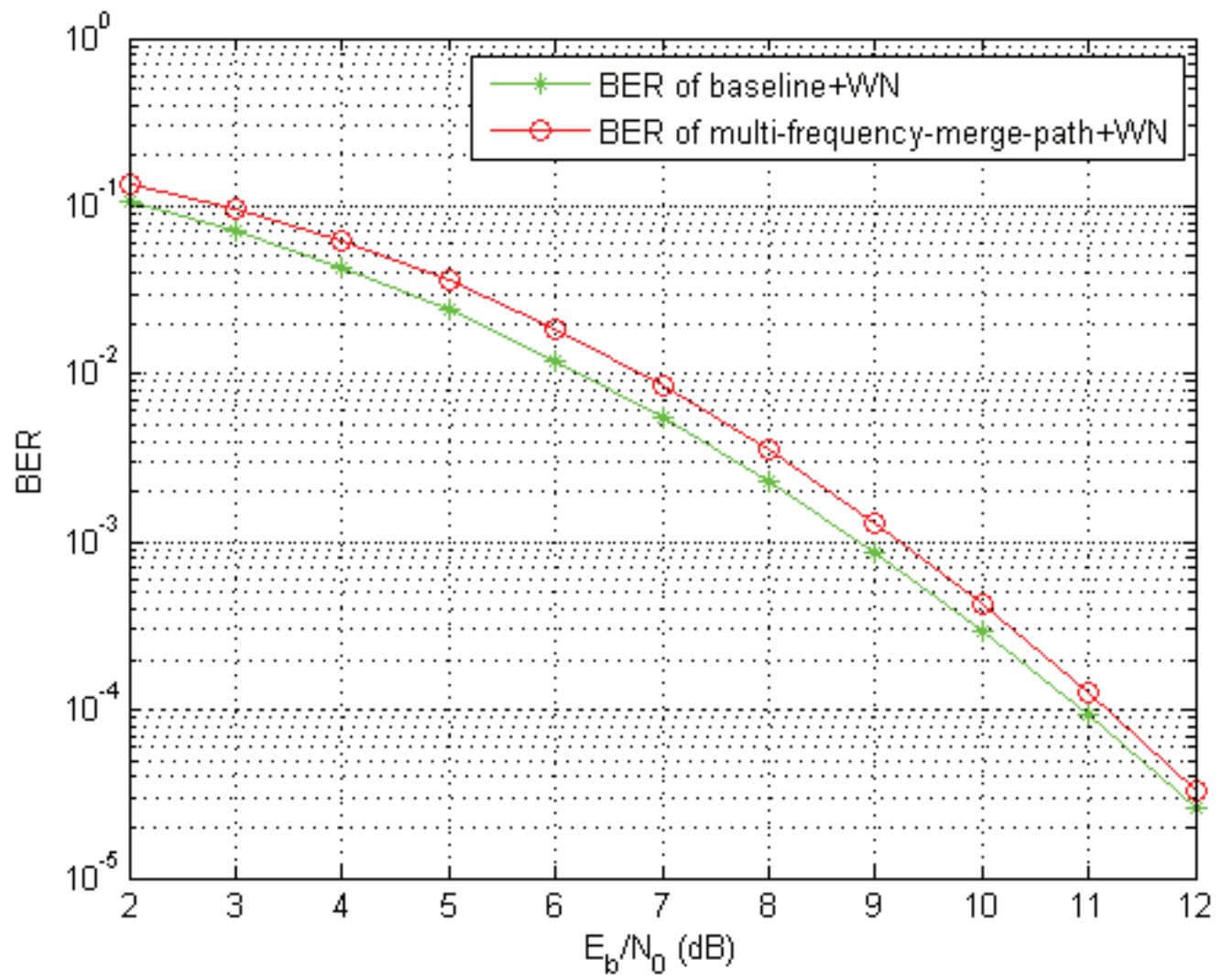

Figure 7.5: The BER of the baseline model vs. the BER of the multi-frequencymerge-path model.

one with asterisks is the BER curve derived from the baseline model. Both of them are with AWGN noise. The BER values with the multi-frequency-merge-path model are slightly larger than the BER values with the baseline model.

\section{Multi-Frequency-Pressure-Sum}

Figure 7.6 shows three BER versus $E_{b} / N_{0}$ curves. The $x$-axis represents the $E_{b} / N_{0}$ ratio. The $y$-axis corresponds to the BER values. The blue dashed curve is the theoretical BER curve of BPSK modulation [46]. The red curve with asterisks is the BER curve derived from the simulation of the multi-frequency-pressure-sum model adding AWGN noise (WN). The magenta curve with circles is the BER curve derived from the simulation of the multi-frequency-pressure-sum model adding colored noise 


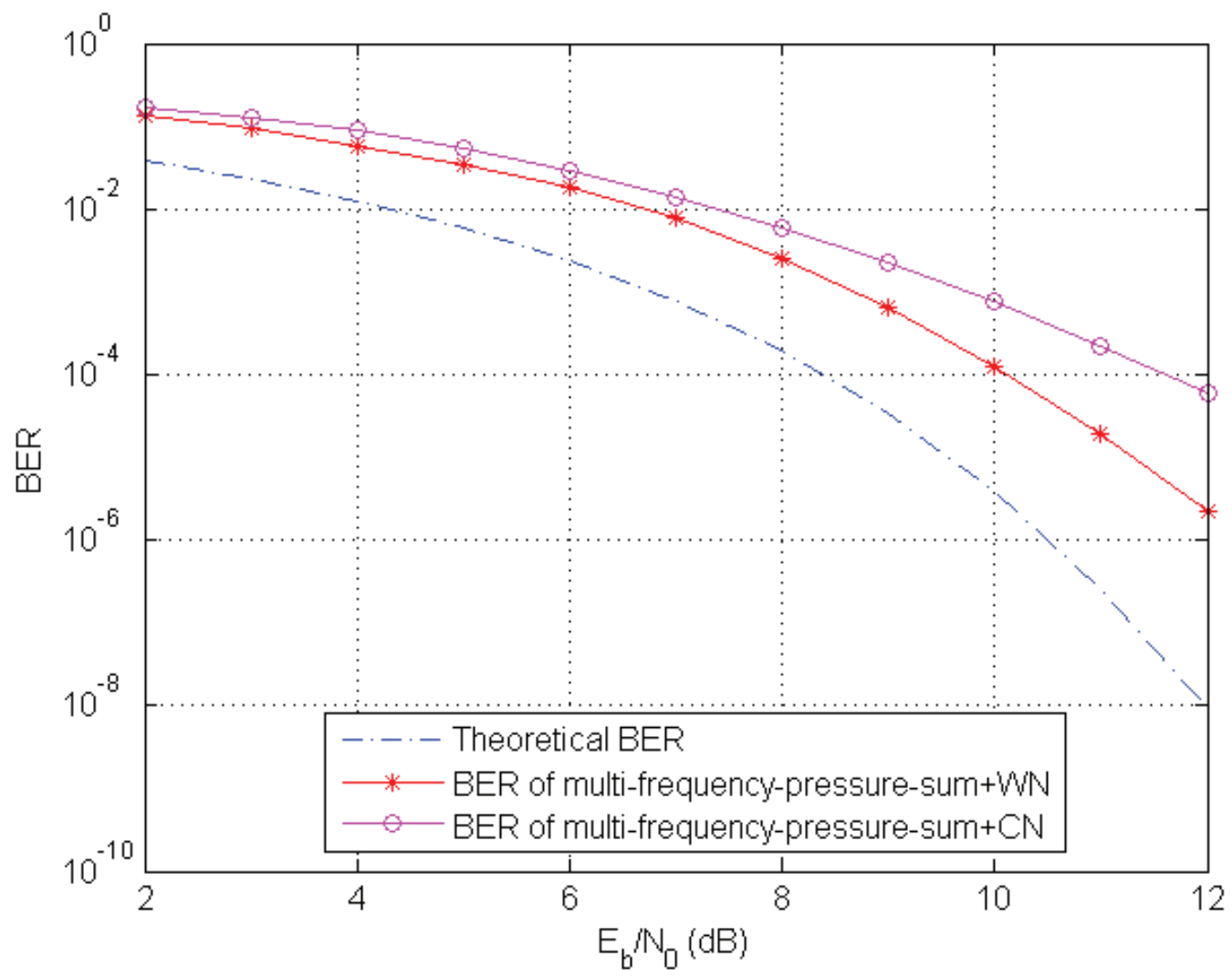

Figure 7.6: Theoretical BER of BPSK modulation vs. the BER of the multifrequency-pressure-sum model.

$(\mathrm{CN})$.

The simulation results show that the BERs of the multi-frequency-pressure-sum model, with noise, are higher than the theoretical BERs. With colored noise, the BERs are larger than with AWGN noise. It is due to the frequency dependent feature of colored noise, which means that colored noise has more impact on the signal. When the $E_{b} / N_{0}$ ratio increases, i.e., the signal-to-noise ratio rises, the gap between the BER of the multi-frequency-pressure-sum + WN curve and multi-frequency-pressure-sum + $C N$ curve are larger. The BERs with AWGN noise decrease more quickly than those with colored noise.

The BER curve of the multi-frequency-pressure-sum is also compared with the one of the baseline model in Figure 7.7. The red curve with circles is the BER curve 


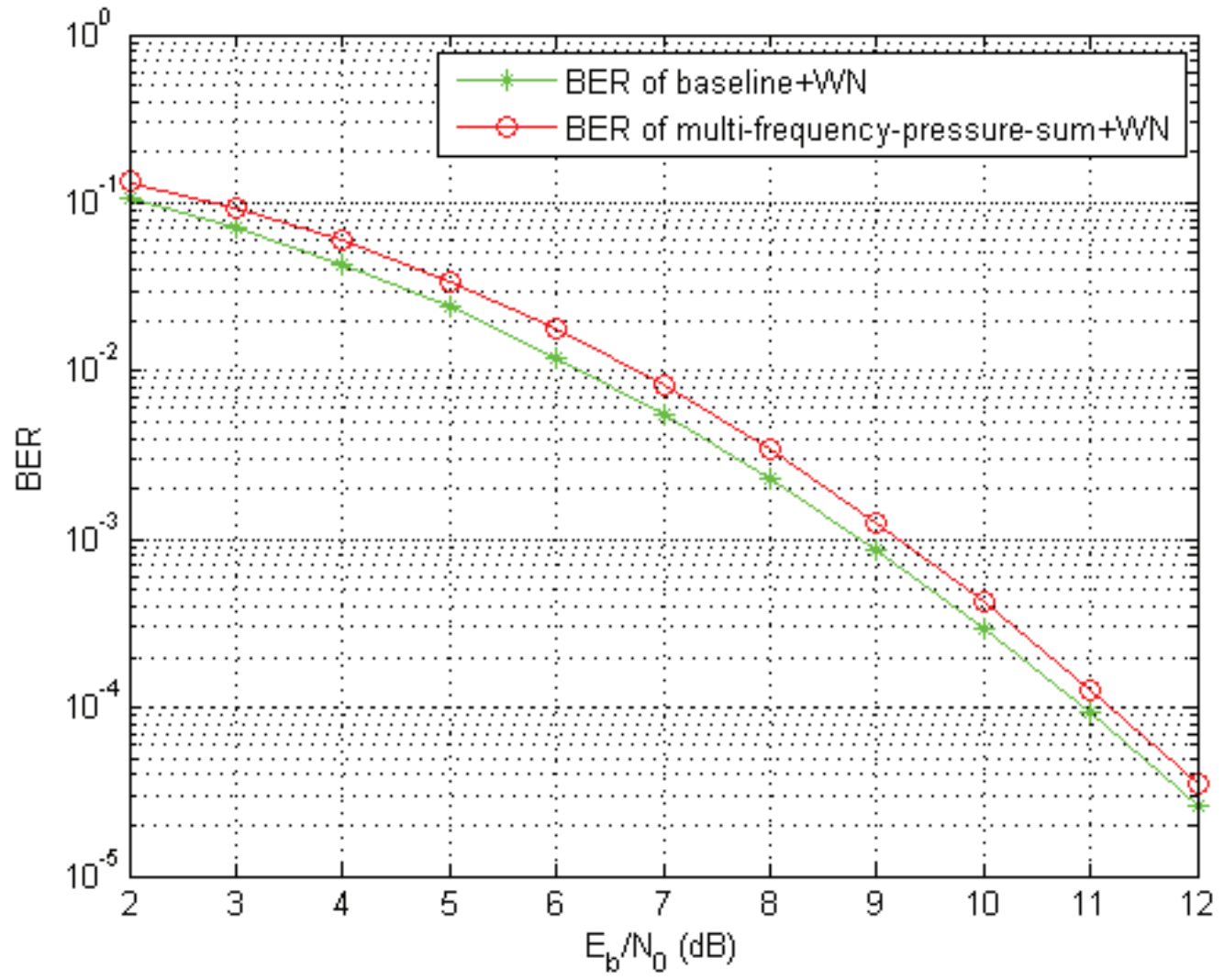

Figure 7.7: The BER of the baseline model vs. the BER of the multi-frequencypressure-sum model.

acquired from the simulation of the multi-frequency-pressure-sum model. The green one with asterisks is the BER curve derived from the baseline model. Both of them are with AWGN noise. The BER values with the multi-frequency-pressure-sum model are slightly larger than the BER values with the baseline model.

\section{Multi-Frequency-Merge-Path Down Conversion}

Figure 7.8 shows three BER versus $E_{b} / N_{0}$ curves. The $x$-axis represents the $E_{b} / N_{0}$ ratio. The $y$-axis corresponds to the BER values. The blue dashed curve is the theoretical BER curve of BPSK modulation [46]. The red curve with asterisks is the BER curve derived from the simulation of the multi-frequency-merge-path down conversion model adding AWGN noise (WN). The magenta curve with circles is the BER 


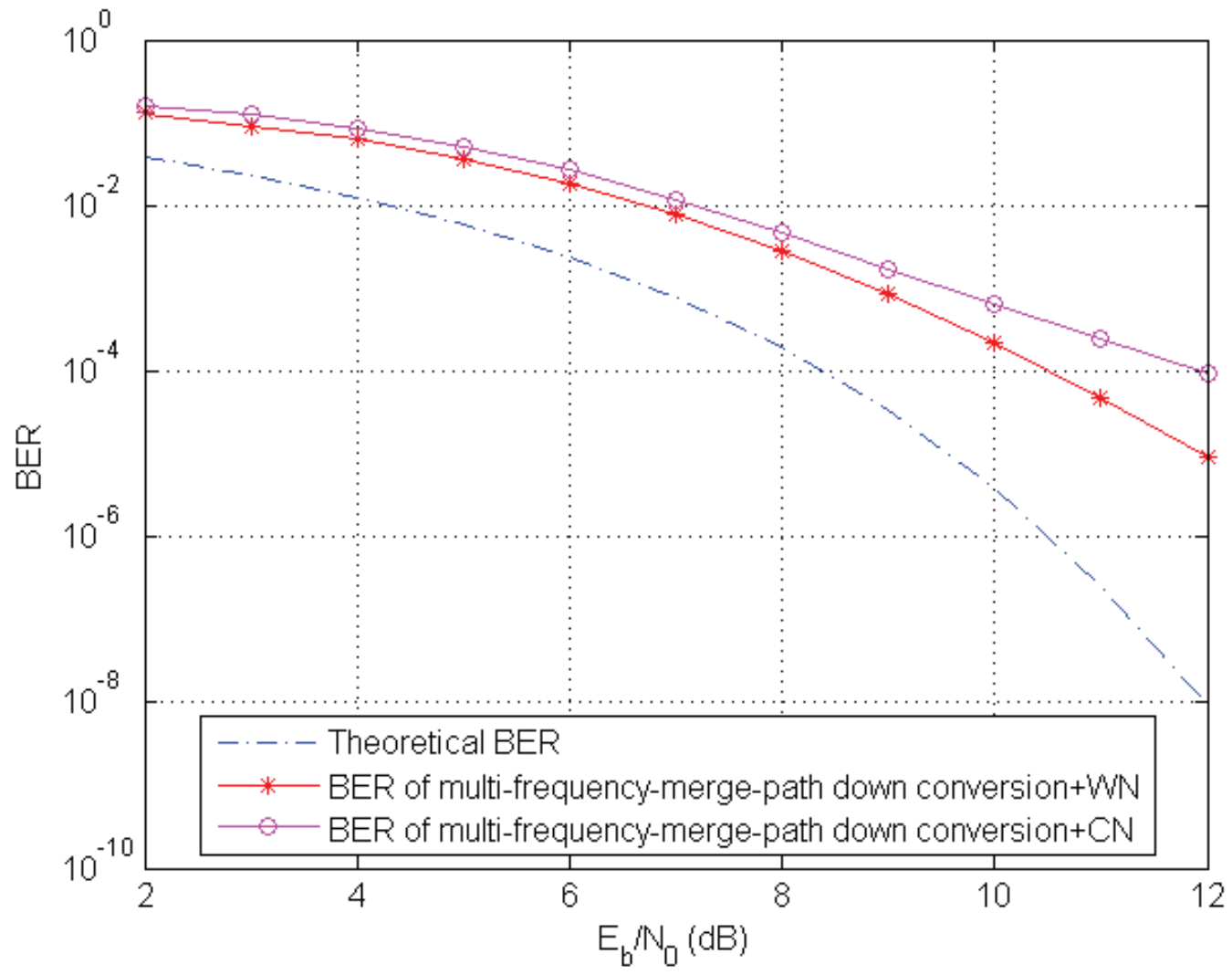

Figure 7.8: Theoretical BER of BPSK modulation vs. the BER of the multifrequency-merge-path down conversion model.

curve derived from the simulation of the multi-frequency-merge-path down conversion model adding colored noise $(\mathrm{CN})$.

The simulation results show that the BERs of the multi-frequency-merge-path down conversion model, with noise, are higher than the theoretical BERs. With colored noise, the BERs are larger than with AWGN noise. It is due to the frequency dependent feature of colored noise, which means that colored noise has more impact on the signal. When the $E_{b} / N_{0}$ ratio increases, i.e., the signal-to-noise ratio rises, the gap between the BER of the multi-frequency-merge-path down conversion $+W N$ curve and multi-frequency-merge-path down conversion $+C N$ curve are larger. The BERs with AWGN noise decrease more quickly than those with colored noise. 


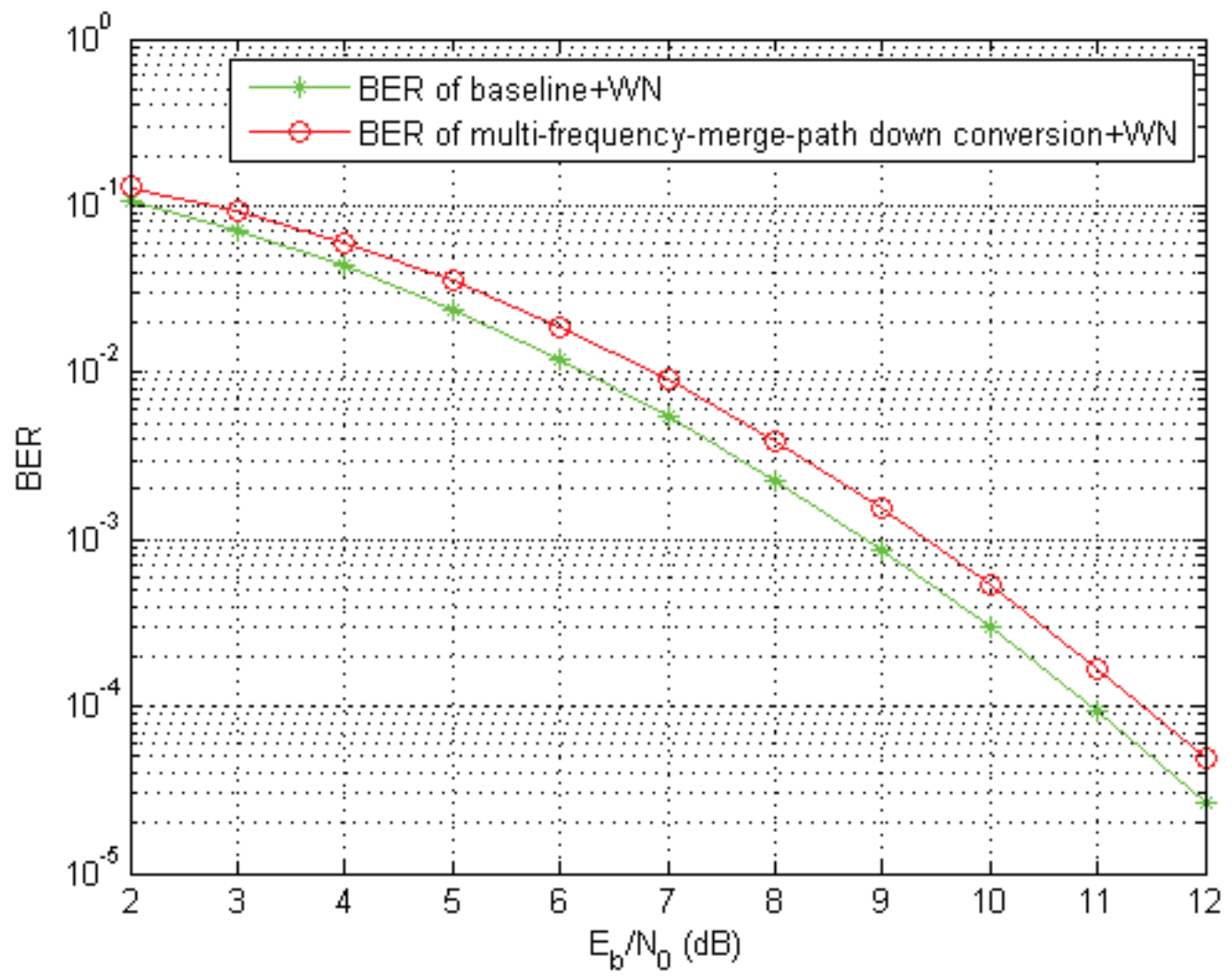

Figure 7.9: The BER of the baseline model vs. the BER of the multi-frequencymerge-path down conversion model.

The BER curve of the multi-frequency-merge-path down conversion is also compared with the one of the baseline model in Figure 7.9. The red curve with circles is the BER curve acquired from the simulation of the multi-frequency-merge-path down conversion model. The green one with asterisks is the BER curve derived from the baseline model. Both of them are with AWGN noise. The BER values with the multi-frequency-merge-path down conversion model are slightly larger than the BER values with the baseline model.

\section{Linear Fitting}

Figure 7.10 shows three BER versus $E_{b} / N_{0}$ curves. The $x$-axis represents the $E_{b} / N_{0}$ ratio. The $y$-axis corresponds to the BER values. The blue dashed curve is the 


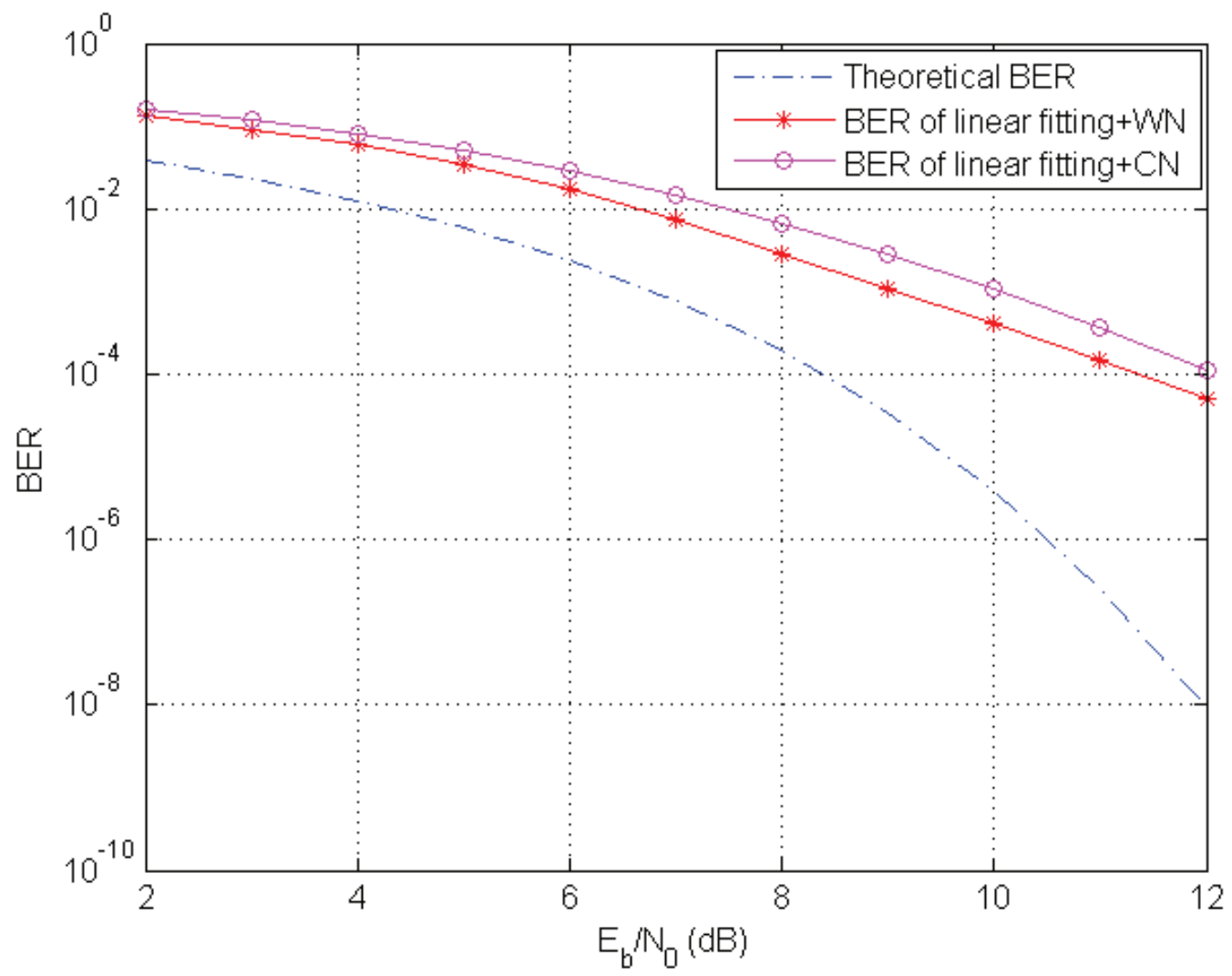

Figure 7.10: Theoretical BER of BPSK modulation vs. the BER of the linear fitting model.

theoretical BER curve of BPSK modulation [46]. The red curve with asterisks is the BER curve derived from the simulation of the linear fitting model adding AWGN noise (WN). The magenta curve with circles is the BER curve derived from the simulation of the linear fitting model adding colored noise $(\mathrm{CN})$.

The simulation results show that the BERs of the linear fitting model, with noise, are higher than the theoretical BERs. With colored noise, the BERs are larger than with AWGN noise. It is due to the frequency dependent feature of colored noise, which means that colored noise has more impact on the signal. When the $E_{b} / N_{0}$ ratio increases, i.e., the signal-to-noise ratio rises, the gap between the BER of the linear fitting $+W N$ curve and linear fitting $+C N$ curve are larger. The BERs with AWGN noise decrease more quickly than those with colored noise.

The BER curve of the linear fitting model is also compared with the one of the 


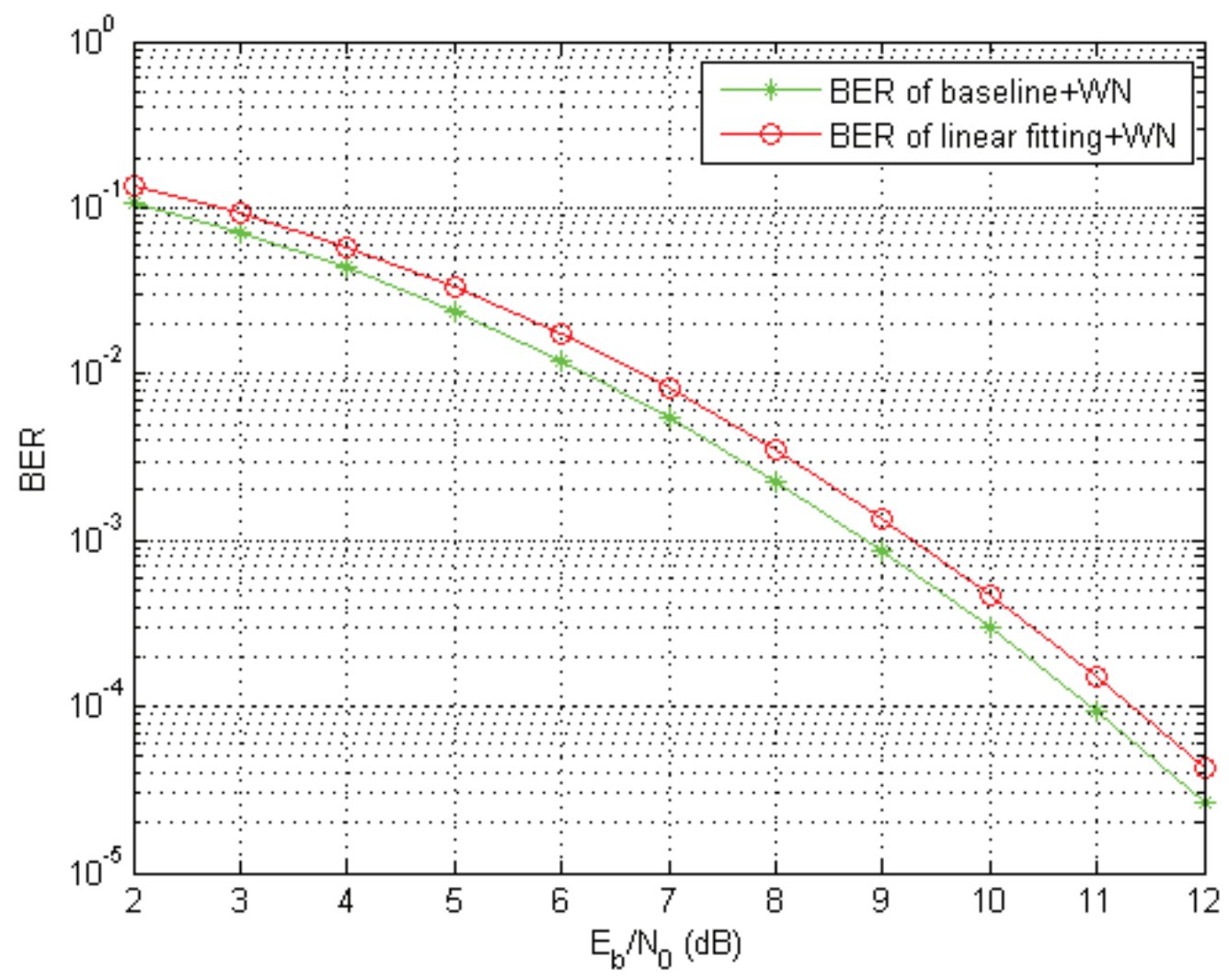

Figure 7.11: The BER of the baseline model vs. the BER of the linear fitting model.

baseline model in Figure 7.11. The red curve with circles is the BER curve acquired from the simulation of the linear fitting model. The green one with asterisks is the BER curve derived from the baseline model. Both of them are with AWGN noise. The BER values with the multi-frequency-merge-path model are slightly larger than the BER values with the baseline model.

\subsubsection{Coherent}

\section{Coherent Multi-Frequency-Merge-Path}

Figure 7.12 shows three BER versus $E_{b} / N_{0}$ curves. The $x$-axis represents the $E_{b} / N_{0}$ ratio. The $y$-axis corresponds to the BER values. The blue dashed curve is the theoretical BER curve of BPSK modulation [46]. The red curve with asterisks is the BER curve derived from the simulation of the coherent multi-frequency-merge-path 


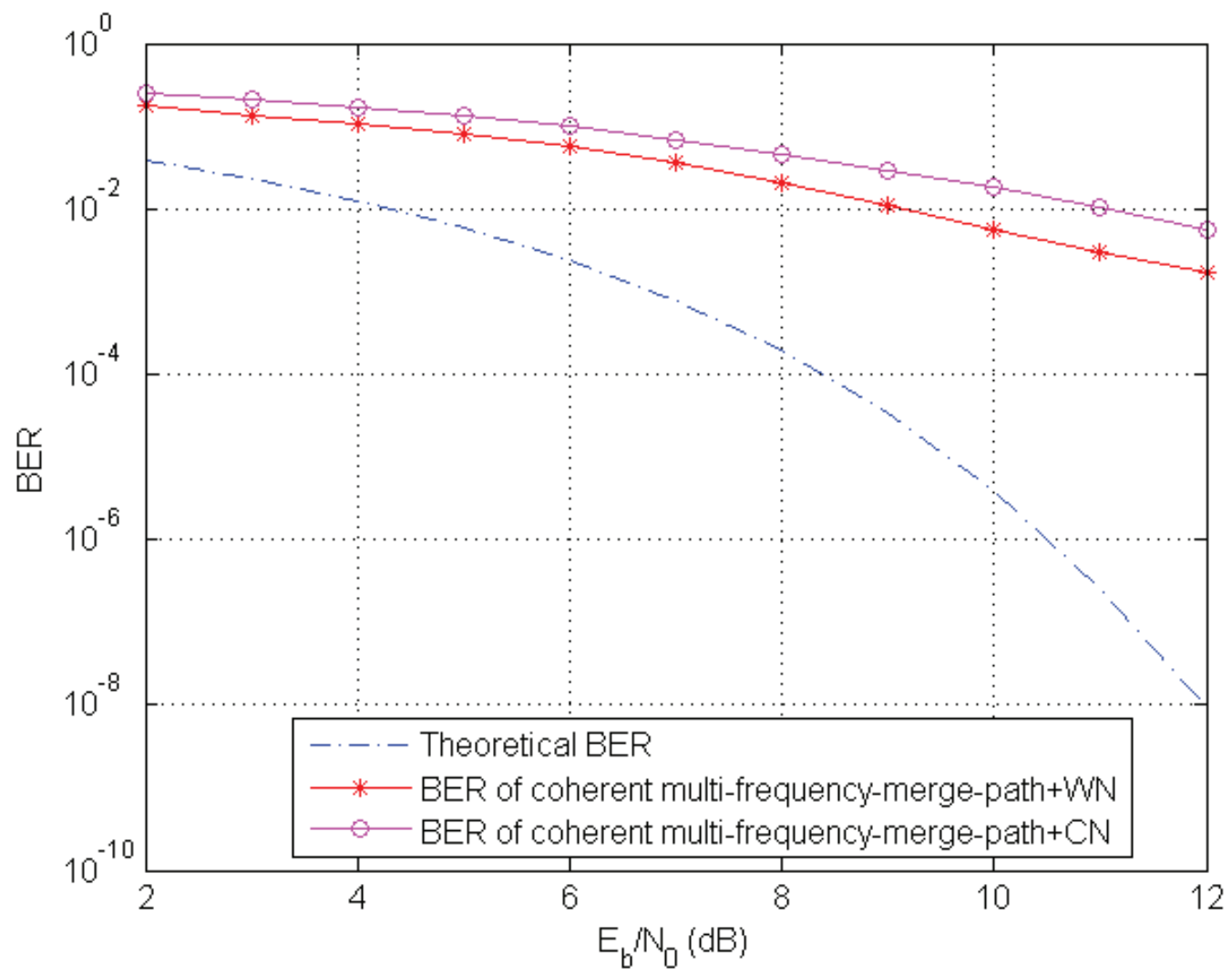

Figure 7.12: Theoretical BER of BPSK modulation vs. the BER of the coherent multi-frequency-merge-path model.

model adding AWGN noise (WN). The magenta curve with circles is the BER curve derived from the simulation of the coherent multi-frequency-merge-path model adding colored noise $(\mathrm{CN})$.

The simulation results show that the BERs of the coherent multi-frequency-mergepath model, with noise, are higher than the theoretical BERs. With colored noise, the BERs are larger than with AWGN noise. It is due to the frequency dependent feature of colored noise, which means that colored noise has more impact on the signal. When the $E_{b} / N_{0}$ ratio increases, i.e., the signal-to-noise ratio rises, the gap between the BER of the coherent multi-frequency-merge-path $+W N$ curve and coherent multifrequency-merge-path $+C N$ curve are larger. The BERs with AWGN noise decrease more quickly than those with colored noise. However, because the attenuation of this 


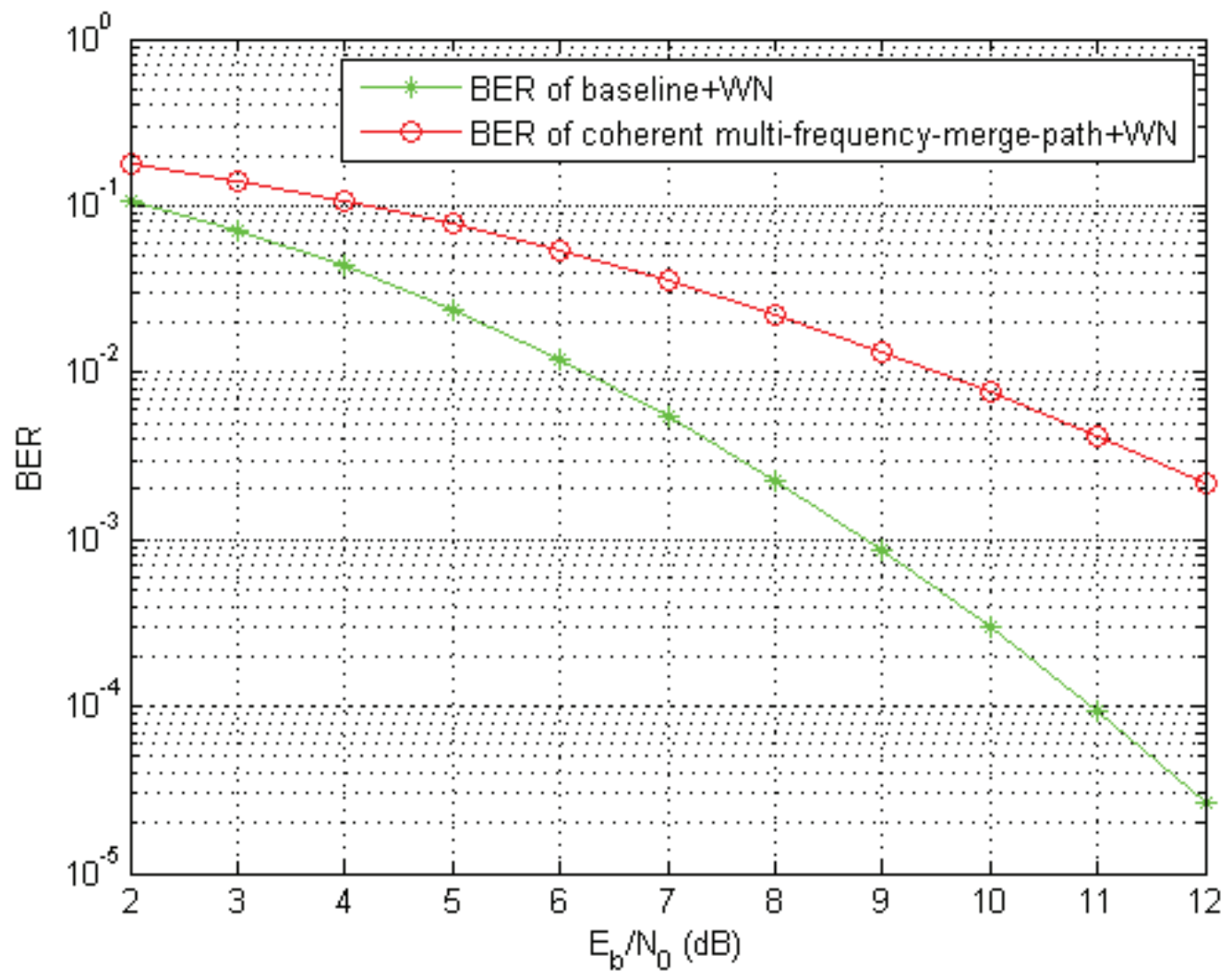

Figure 7.13: The BER of the baseline model vs. the BER of the coherent multifrequency-merge-path model.

coherent model is bigger than all the incoherent models, the noise has less effects on the BER. Hence, the BER decreasing rate with the rising of the $E_{b} / N_{0}$ ratio in this model is lower than in incoherent models.

The BER curve of the coherent multi-frequency-merge-path is also compared with the one of the baseline model in Figure 7.13. The red curve with circles is the BER acquired from the simulation of the coherent multi-frequency-merge-path model. The green one with asterisks is the BER curve derived from the baseline model. Both of them are with AWGN noise. The BER values with the coherent multi-frequencymerge-path model are larger than the BER values with the baseline model. When the $E_{b} / N_{0}$ ratio becomes larger, the attenuation calculation increases the differences between the two models. 


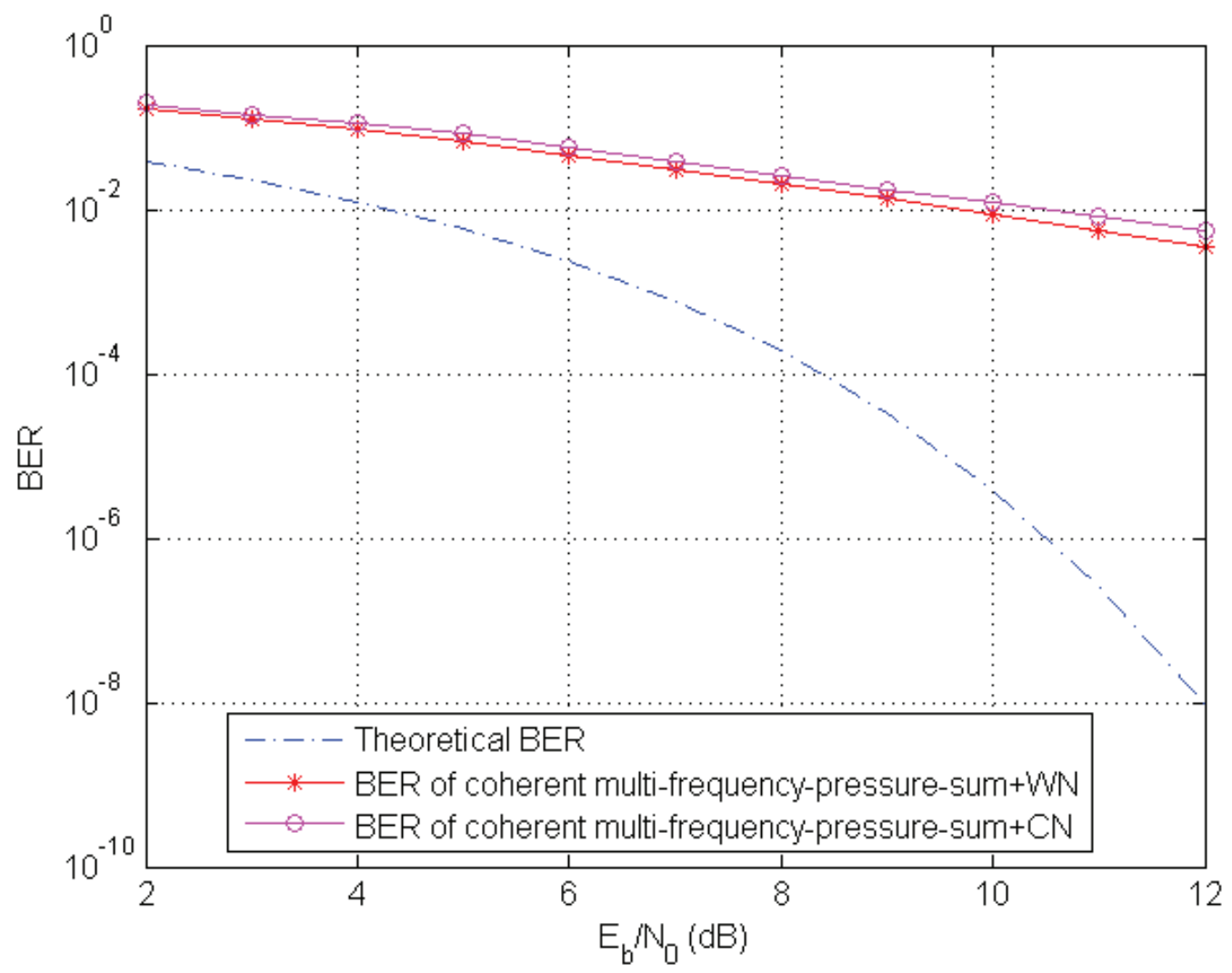

Figure 7.14: Theoretical BER of BPSK modulation vs. the BER of the coherent multi-frequency-pressure-sum model.

\section{Coherent Multi-Frequency-Pressure-Sum}

Figure 7.14 shows three BER versus $E_{b} / N_{0}$ curves. The $x$-axis represents the $E_{b} / N_{0}$ ratio. The $y$-axis corresponds to the BER values. The blue dashed curve is the theoretical BER curve of BPSK modulation [46]. The red curve with asterisks is the BER curve derived from the simulation of the coherent multi-frequency-pressuresum model adding AWGN noise (WN). The magenta curve with circles is the BER curve derived from the simulation of the coherent multi-frequency-pressure-sum model adding colored noise $(\mathrm{CN})$.

The simulation results show that the BERs of the coherent multi-frequencypressure-sum model, with noise, are higher than the theoretical BERs. With colored 
noise, the BERs are larger than with AWGN noise. It is due to the frequency dependent feature of colored noise, which means that colored noise has more impact on the signal. When the $E_{b} / N_{0}$ ratio increases, i.e., the signal-to-noise ratio rises, the gap between the BER of the coherent multi-frequency-pressure-sum $+W N$ curve and coherent multi-frequency-pressure-sum $+C N$ curve are larger. The BERs with AWGN noise decrease more quickly than those with colored noise. However, because the attenuation of this coherent model is larger than for all the incoherent models, the noise has less effects on the BER. Hence, the BER as a function of the $E_{b} / N_{0}$ ratio is decreasing slower in this model, with respect to the incoherent models.

The BER curve of the coherent multi-frequency-pressure-sum is also compared with the one of the baseline model in Figure 7.15. The red curve with circles is the BER acquired from the simulation of the coherent multi-frequency-pressure-sum model. The green one with asterisks is the BER curve derived from the baseline model. Both of them are with AWGN noise. The BER values with the coherent multi-frequency-pressure-sum model are larger than the BER values with the baseline model. When the $E_{b} / N_{0}$ ratio becomes larger, the attenuation calculation increases the differences between the two models.

With coherent attenuation, the BER becomes a positive constant, instead of zero, when the noise drops down below a certain threshold. The exact threshold depends on the coherent model. The coherent attenuation is irregular when the frequency changes because of the interference created by multipath attenuation. Therefore, the signal is damaged by the coherent attenuation, which causes communication errors. For example, the BER curve of the coherent multi-frequency-pressure-sum model, in the range 2 to $50 \mathrm{~dB}$, is shown as Figure 7.16. When the $E_{b} / N_{0}$ ratio is larger than $25 \mathrm{~dB}$, the BERs stay at about $8 \cdot 10^{-5}$. The errors are due to multipath propagation.

\subsection{Comparison With the Experimental Data}

A sea trial was performed by the DRDC Atlantic Research Center [7] using Teledyne Benthos Acoustic Modems [53, 52]. Four nodes were installed in the Bedford Basin (N.S., Canada). Using the data resulting from the sea trial, we have a plot for the BER versus $E_{b} / N_{0}$. The experiment used Frequency Shift Keying (FSK) modulation 


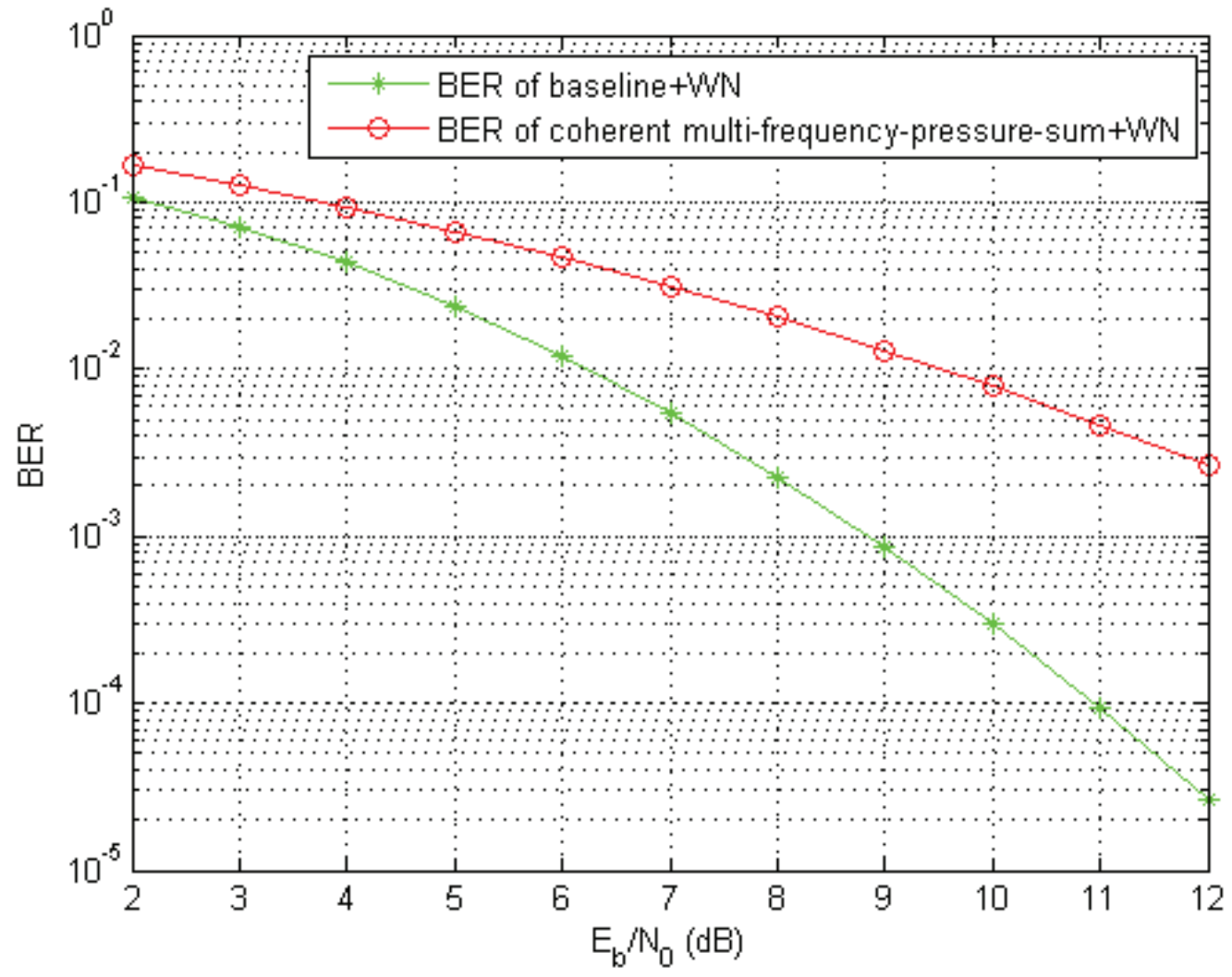

Figure 7.15: The BER of the baseline model vs. the BER of the coherent multifrequency-pressure-sum model. 


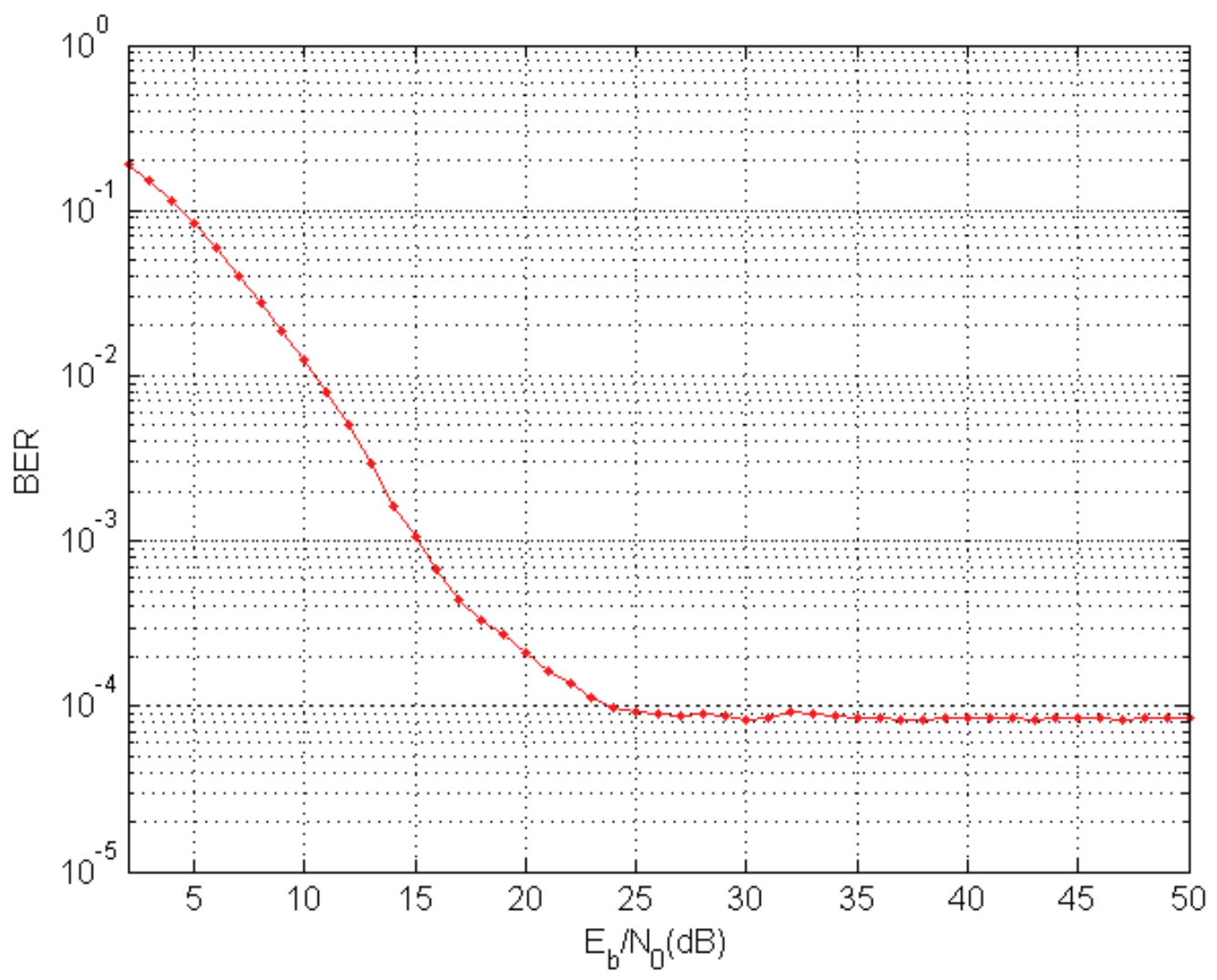

Figure 7.16: The BER of the coherent multi-frequency-pressure-sum model with colored noise. 
[46]. The results of the experiment have been compared with the ones of all the aforementioned models. The BERs of the coherent multi-frequency-pressure-sum model are the closest to the BERs of the experimental data.

Figure 7.17 shows a comparison of the BER curve obtained from the simulation of the coherent multi-frequency-pressure-sum model and experimental data. Three BER versus $E_{b} / N_{0}$ curves are presented. The $x$-axis represents the $E_{b} / N_{0}$ ratio in $\mathrm{dB}$ form. The $y$-axis corresponds to the BER values. The blue solid line is the BER curve of the theoretical reference for FSK modulation. The red circles are the experimental data points. The red solid line is the BER of the experimental data modeled with exponential curve fitting of MATLAB. The green solid line with asterisks represents the BER of the coherent multi-frequency-pressure-sum model with colored noise and Multiple FSK (MFSK) modulation. The BER curves of the experimental data with curve fitting and coherent multi-frequency-pressure-sum model care very similar, but not identical. The BERs of the coherent multi-frequency-pressure-sum model are a little lower than those of the experiment data. The possible reason is that the environment is not exactly the same as the setting in the simulation.

Two statistical tests, paired-sample t-test and two sample Kolmogorov-Smirnov test [5], are used to verify if the BER curve of the coherent multi-frequency-pressuresum model has the same distribution as the experimental BER curve. The t-test is available in MATLAB as function ttest $(\mathrm{x}, \mathrm{y})$. The null hypothesis of ttest $(\mathrm{x}, \mathrm{y})$ is that the difference between random variables $\mathrm{x}$ and $\mathrm{y}$, i.e., $\mathrm{x}-\mathrm{y}$, follows a normal distribution with zero mean [30]. The two sample Kolmogorov-Smirnov test is available in MATLAB as function kstest2 $(\mathrm{x}, \mathrm{y})$. The null hypothesis of kstest2 $(\mathrm{x}, \mathrm{y})$ is that the difference between random variables $\mathrm{x}$ and $\mathrm{y}$, i.e., $\mathrm{x}-\mathrm{y}$, follows a normal distribution with zero mean [30]. Both t-test and Kolmogorov-Smirnov test fail to reject the null hypothesis at the $5 \%$ significance level. It means that the BER curves of the coherent multi-frequency-pressure-sum model and the experimental data in Figure 7.17 have the same probability distribution. 


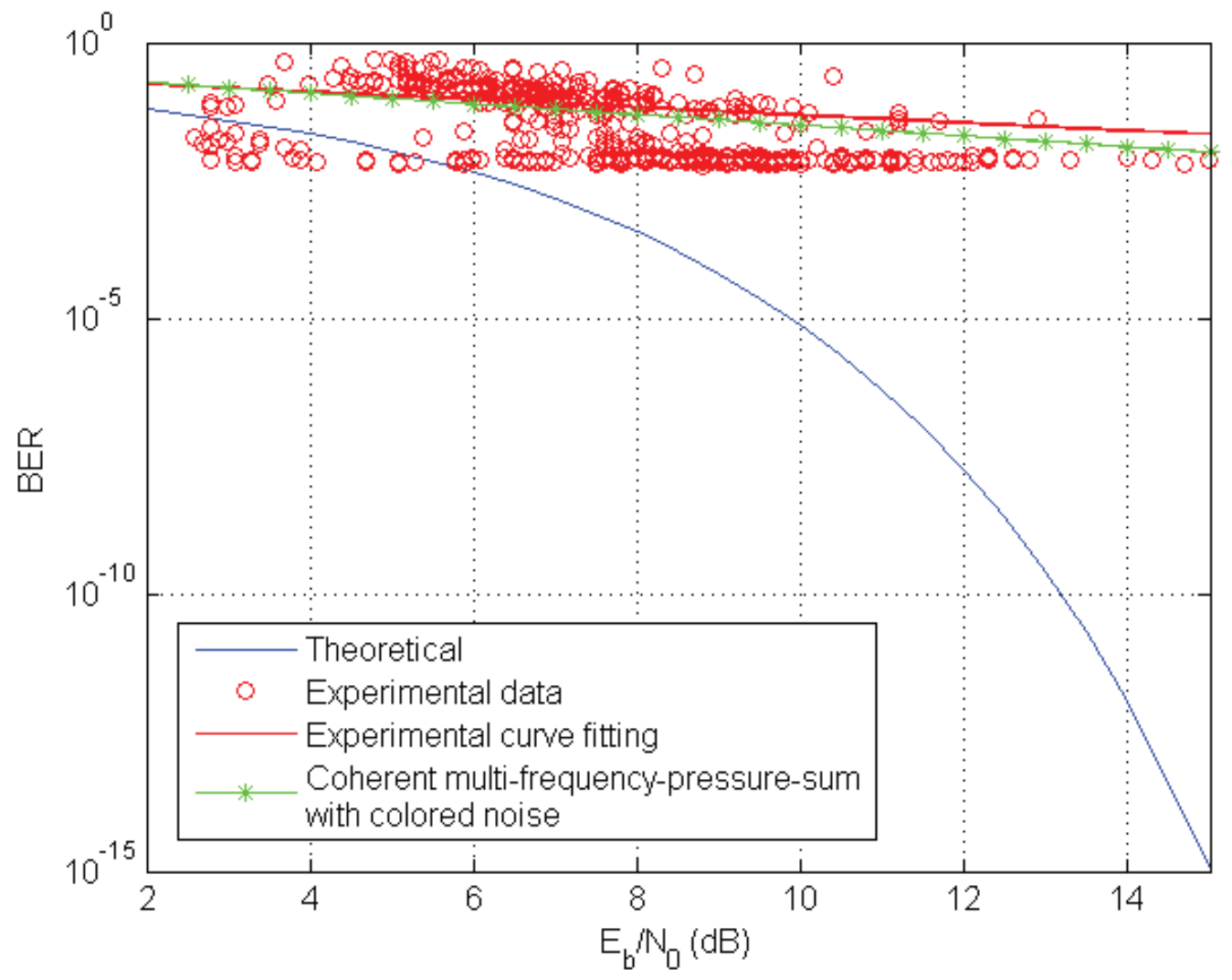

Figure 7.17: The BER comparison of experimental data vs. the coherent multifrequency-pressure-sum model. 


\subsection{Summary}

In this chapter, eight models are simulated, covering the time domain and frequency domain models attenuated by incoherent or coherent loss with white or colored noise. According to the simulation results, the following conclusions can be drawn:

1. All models, in time domain or in frequency domain, have higher BERs, i.e., worse performance, than the theoretical reference. It is due to the fact that the theoretical reference takes solely into account AWGN.

2. Similar, but not identical, simulation results can be obtained using incoherent models.

3. The BER values with all incoherent models are slightly larger than the BER values with the baseline model. All BERs of the coherent models are larger than the ones of the baseline model.

4. The models with coherent attenuation result in worse performance, in terms of BERs, than the models with incoherent attenuation.

5. Colored noise impacts the system performance more than white noise for all models, due to the frequency dependent feature of colored noise. For the incoherent models, when the $E_{b} / N_{0}$ ratio rises, the BERs with AWGN noise decrease more quickly than those with colored noise. However, for the coherent models with colored noise, the BER is falling almost the same as for the coherent models with white noise. The attenuation of the coherent models is larger than for call the incoherent models. The noise has less effects on the BERs in the coherent models than in the incoherent models.

6. The coherent attenuation is such that the BERs of coherent models cannot drop down to zero, even if the noise decreases to zero. The BERs of coherent models are maintained around a certain value after the $E_{b} / N_{0}$ ratio is higher than a threshold.

7. The BER curve of the coherent multi-frequency-pressure-sum model with colored noise is the most similar to the BER curve of the field experimental data. 
However, the BER curves are not identical because the environments are different. The statistical tests verify that these two BER curves have the same distribution. 


\section{Chapter 8}

\section{Conclusions and Future Work}

\subsection{Conclusions}

In this thesis, eight underwater communication models are proposed, simulated and compared. These models consider multi-path attenuation and ambient noise. They are divided into two groups. One group is in the time domain. The other is in the frequency domain. All approaches take into account attenuation along with white or colored noise. Both incoherent and coherent attenuation are considered. Incoherent attenuation means that the interference created by the phase differences is ignored. Coherent attenuation means that the phase differences of signals, propagating through different paths, are taken into account. In the time domain, two models are proposed. In the frequency domain, four incoherent models and two coherent models are implemented. White noise is modeled as traditional Additive White Gaussian Noise (AWGN). Colored noise makes the white noise go through a low-pass filter with a profile similar to the one of the underwater ambient noise. The simulations are implemented using MATLAB and the BELLHOP tracing program. The metric bit error rate versus energy per bit to noise power spectral density ratio $E_{b} / N_{0}$ is applied for evaluation. All models are compared with a theoretical reference and a baseline method. The coherent models with colored noise in frequency domain are more similar to the situation of real environment.

For both groups, time domain and frequency domain, all models have higher BERs, i.e., worse performance, than the theoretical reference. It is due to the fact that the theoretical reference takes solely into account AWGN.

The models with coherent attenuation result in worse performance, in terms of BERs, than the models with incoherent attenuation. The incoherent simulation results are similar, but not identical. All incoherent models have simulation results close to the ones of the baseline model, when the signal-to-noise ratio is low. Due 
to the lower relative effects of noise, the BERs of the incoherent models are larger than the ones of the baseline model. All BERs of the coherent models are larger than the ones of the baseline model. The coherent attenuation is such that the BERs of coherent models cannot drop down to zero, even if the noise is null. The BERs of coherent models are maintained around a certain value when the $E_{b} / N_{0}$ ratio is higher than a threshold.

Colored noise impacts the system performance more than white noise for all models, due to the frequency dependent feature of colored noise. For the incoherent models, when the $E_{b} / N_{0}$ ratio rises, the BERs with AWGN decrease more steeply than with colored noise. However, for the coherent models with colored noise, the BER is falling almost the same as for the coherent models with white noise. The attenuation of the coherent models is larger than for all the incoherent models. The noise has less effects on the BERs in the coherent models than in the incoherent models.

Experimental data are also used to evaluate the models. The BER curve of the coherent multi-frequency-pressure-sum model with colored noise is the most similar to the BER curve of the field experimental data. However, the BER curves are not identical because the environments are different. Statistical tests verify that these two BER curves have the same distribution.

\subsection{Future Work}

Future work will focus on the time-varying model, Orthogonal Frequency Division Multiplexing (OFDM) modulation and parallel computing. Time-varying modeling is used when a transmitter or receiver is mobile. The Doppler effect has to be taken into account. It changes the frequency of the received signal due to the changes of distance between the transmitter and receiver. In time-varying modeling, multipath causes not only intersymbol interference (ISI) but also frequency shift [3]. OFDM is based on multicarrier modulation, using orthogonal subcarriers to carry data. It has been proven robust and much appropriate in underwater environment because it offers low complexity design [39]. Parallel computing is a promising technique to accelerate computation in underwater acoustic communication. Some parallel architectures, 
such as Compute Unified Device Architecture (CUDA) and General Purpose Unit (GPU), can be used for ray tracing or normal mode model [26]. 


\section{Appendix A}

\section{Experiment environment}

The implementation has been done using MATLAB and the BELLHOP ray tracing program. A BELLHOP-MATLAB wrapper is supplied by Maritime Way Scientific, which provides the interfaces to call Bellhop functions. The modulation/demodulation and noise generation are based on the work of Borowski. The key parameters used in the experiments shows as the following table: All parameters are written to a en-

Table A.1: Key Parameters

\begin{tabular}{|l|l|}
\hline Source frequency & $19^{\sim} 21$ kilohertz \\
\hline Sound speed profile & isospeed: 1500 meter/second \\
\hline Source depth & 50 meter \\
\hline Received depth & 100 meter \\
\hline Maximum depth & 200 meter \\
\hline Launching angle & $-45^{\sim} 45$ degree \\
\hline
\end{tabular}

vironmental file used by BELLHOP software. The environmental file (ending with the .env extension) follows the rule in the BELLHOP manual $[41,35]$. An example of environmental file is shown as below: 
Table A.2: Environmental file

'APL:muddy sand/'
20000.00 ! Frequency (Hz)
1 ! NMedia
'CVFT' ! Top Option
00.00200 .00 ! Nmesh sigma depth
0.001500 .00 / ! z c
200.001500 .00 / ! z c
'A ' 0.00 ! Bottom Option, sigma
200.001620 .000 .001 .340 .580 .00 / ! lower halfspace
1 ! NSD
50.00 / ! SD(1:NSD) (m)
1 ! NRD
100.00 / ! RD(1:NRD) (m)
1 ! NRR
1.50 / ! RR(1:NRR) (km)
'E' ! Run Type
$0 !$ Nbeams
-45.000000 45.000000 / ! angles (degrees)
$0.000000220 .0000001 .515000 !$ deltas (m) Box.z (m) Box.r




\section{Appendix B}

\section{Incoherent, coherent and acoustic pressure calculation interface in BELLHOP}

We can set up different run-type parameters to obtain various values. The run-type parameters are shown in the following table [41].

Table B.1: Run-type parameters

The run-type parameters can correspond to:

'A': calculate amplitudes and travel times;

'E': calculate eigenray coordinates;

' $R$ ': calculate ray coordinates;

'C': calculate coherent acoustic pressures;

'I': calculate incoherent acoustic pressures;

'S': calculate semi-coherent acoustic pressures.

\section{B.1 Incoherent Interface}

The incoherent attenuation within a specific range can be acquired from the generated shade file using BELLHOP when setting run-type in environment file as 'I', shown in Table A.2. The following BELLHOP function reads all the information, including the incoherent acoustic pressure, from a shade file:

[PlotTitle, PlotType,freq,atten,Pos,pressure $]=$ read_shd('filename.shd' $)$;

The meaningful return values are Pos and pressure. Pos includes launching angles, source depth, receiver depth and receiver range. pressure is a four-dimension matrix, where the dimensions are the number of launching angles, the number of source depth, the number of receiver depth and the number of receiver range, separately. 


\section{B.2 Coherent Interface}

To obtain coherent acoustic pressure, the BELLHOP interface is very similar to the incoherent interface, except that the run-type in environment file is ' $\mathrm{C}$ ' instead of ' $\mathrm{I}$ ', shown in Table A.2. The results are contained in a shade file. The reading of the shade file is the same as in Section B.1.

\section{B.3 Acoustic Pressure Interface}

The attenuation of each eigenray can be acquired from the generated arrival file using BELLHOP when setting run-type in the environment file as ' $\mathrm{A}$ ', as shown in Table A.2. We can use the function read_arrivals_bin() in BELLHOP to get the Pos and Attenuation from the file.

$$
\left.[\text { Arr }, \text { Pos } S R]=\text { read_arrivals_bin('filename.arr' }{ }^{\prime}\right)
$$

Returned value Arr includes the attenuations, delays, source angles, received angles, the number of times to hit the top and the number of times to hit the bottom for each eigenray in a certain distance. Returned value Pos $R$ consists of number and values of source depth, receiver depth and receiver range. 


\section{Bibliography}

[1] Ocean. http://en.wikipedia.org/wiki/Ocean, May 2015.

[2] M. A. Ainslie and J. G. McColm. A simplified formula for viscous and chemical absorption in sea water. The Journal of the Acoustical Society of America, 103(3):1671-1672, 1998.

[3] I. F. Akyildiz, D. Pompili, and T. Melodia. Challenges for efficient communication in underwater acoustic sensor networks. ACM Sigbed Review, 1(2):3-8, 2004 .

[4] I. F. Akyildiz, D. Pompili, and T. Melodia. Underwater acoustic sensor networks: research challenges. Ad hoc networks, 3(3):257-279, 2005.

[5] M. Barbeau. Wireless Mobile Communications, Networks 8 Security. Available online: http://people.scs.carleton.ca/ barbeau/WirelessBook/index.shtml, 227 pp.

[6] M. Barbeau, S. Blouin, G. Cervera, J. Garcia-Alfaro, B. Hasannezhad, and E. Kranakis. Simulation of underwater communications with a colored noise approximation and mobility. In 28th annual IEEE Canadian Conference on Electrical and Computer Engineering (CCECE), Halifax, Nova Scotia, Canada, May 2015.

[7] S. Blouin. SR acoustic modem parameter - data analysis. Draft report DRDCRDDC-2014-RXXX, 2014.

[8] B. S. Borowski. Application of Channel Estimation to Underwater, Acoustic Communication. PhD thesis, Stevens Institute of Technology, Hoboken, NJ, USA, 2011.

[9] J. B. Bowlin, J. L. Spiesberger, T. F. Duda, and L. F. Freitag. Ocean acoustical ray-tracing software ray. Technical report, DTIC Document, 1992.

[10] M. Chitre, S. Shahabudeen, L. Freitag, and M. Stojanovic. Recent advances in underwater acoustic communications \& networking. In OCEANS 2008, volume 2008, pages 1-10. IEEE, 2008.

[11] M. J. Crocker. Handbook of acoustics. John Wiley \& Sons, 1998.

[12] A. J. Duncan, I. M. Parnum, and P. J. Henley. Efficient modelling of mid to high frequency underwater acoustic propagation. In Proc. Acoustics 2013, Victor Harbor, Australia, 2013. 
[13] B. D. Dushaw and J. A. Colosi. Ray tracing for ocean acoustic tomography. Technical report, DTIC Document, 1998.

[14] P. C. Etter. Underwater acoustic modeling and simulation. CRC Press, 4 edition, 2013.

[15] R. Evans. A coupled mode solution for acoustic propagation in a waveguide with stepwise depth variations of a penetrable bottom. The Journal of the Acoustical Society of America, 74(1):188-195, 1983.

[16] R. Galvin and L. Wang. Measured channel characteristics and the corresponding performance of an underwater acoustic communication system using parametric transduction. IEE Proceedings-Radar, Sonar and Navigation, 147(5):247-253, 2000 .

[17] O. Godin. Wide-angle parabolic equations for sound in a $3 \mathrm{~d}$ inhomogeneous moving medium. In Doklady Physics, volume 47, pages 643-646. Springer, 2002.

[18] R. P. Hodges. Underwater acoustics: Analysis, design and performance of sonar. John Wiley \& Sons, 2011.

[19] J. M. Hovem. Ray trace modeling of underwater sound propagation. In M. G. Beghi, editor, Modeling and Measurement Methods for Acoustic Waves and for Acoustic Microdevices, chapter 23. InTech, 2013.

[20] F. Ingenito, S. Wolf, and J. Miller. Modal acoustic transmission loss (moatl). NRL Report, 8429, 1980.

[21] R. S. Istepanian and M. Stojanovic. Underwater acoustic digital signal processing and communication systems. Springer, 2002.

[22] F. B. Jensen and M. C. Ferla. Snap: The saclantcen normal-mode acoustic propagation model. Technical report, DTIC Document, 1979.

[23] F. B. Jensen, W. A. Kuperman, M. B. Porter, and H. Schmidt. Computational ocean acoustics. Springer Science \& Business Media, 2011.

[24] R. M. Jones, J. P. Riley, and T. M. Georges. Harpo: A versatile three-dimensional hamiltonian ray-tracing program for acoustic waves in an ocean with irregular bottom. Unknown, 1, 1986.

[25] D. B. Kilfoyle, J. C. Preisig, and A. B. Baggeroer. Spatial modulation experiments in the underwater acoustic channel. Oceanic Engineering, IEEE Journal of, 30(2):406-415, 2005. 
[26] M. Lazzarin, P. Casari, and M. Zorzi. Endowing underwater networks with channel awareness: a discussion on computational complexity and information size issues. In Proceedings of Meetings on Acoustics, volume 17, page 070100. Acoustical Society of America, 2014.

[27] X. Lurton. An introduction to underwater acoustics: principles and applications. Springer Science \& Business Media, 2002.

[28] K. V. Mackenzie. Discussion of sea water sound-speed determinations. The Journal of the Acoustical Society of America, 70(3):801-806, 1981.

[29] MathWorks. Fast Fourier Transform (FFT). http://www.mathworks.com/ help/matlab/math/fast-fourier-transform-fft.html/, 2015.

[30] MathWorks. ttest. http://www.mathworks.com/help/stats/ttest.html, 2015.

[31] D. McCammon. Investigation of the transmission loss issue in BELLHOP. http: //cradpdf .drdc-rddc.gc.ca/PDFS/unc84/p531511.pdf, 2008.

[32] T. McDermott. Additive white Gaussian noise (AWGN). In Wireless Digital Communications: Design and Theory, chapter 2, pages 15-26. Tucson Amateur Packet Radio, 1996.

[33] A. Nagl, H. Überall, A. J. Haug, and G. Zarur. Adiabatic mode theory of underwater sound propagation in a range-dependent environment. The Journal of the Acoustical Society of America, 63(3):739-749, 1978.

[34] M. B. Porter. The KRAKEN normal mode program. Technical report, DTIC Document, 1992.

[35] M. B. Porter. The BELLHOP manual and users guide: Preliminary draft. Heat, Light, and Sound Research, Inc., La Jolla, CA, USA, Tech. Rep, 2011.

[36] J. Preisig. Acoustic propagation considerations for underwater acoustic communications network development. ACM SIGMOBILE Mobile Computing and Communications Review, 11(4):2-10, 2007.

[37] J. Proakis and M. Salehi. Introduction. In Digital Communications, chapter 1, pages 10-12. McGraw-Hill Education, 2007.

[38] P. Qarabaqi, Y. M. Aval, and M. Stojanovic. Computationally efficient simulation of underwater acoustic communication systems. In Oceans-San Diego, 2013, pages 1-6. IEEE, 2013. 
[39] A. I. R. Khan, Q. Gang and K. Mehboob. Investigation of channel modeling and simulation of ofdm based communication near northern regions of arabian sea. Research Journal of Applied Sciences, Engineering and Technology, 5(4):11691182, 2013.

[40] K. V. Rangarao and R. K. Mallik. Processing of signals. In Digital Signal Processing: A Practitioner's Approach, chapter 1. John Wiley \& Sons, 2006.

[41] O. C. Rodriguez. General description of the BELLHOP ray tracing program. Physics Department Signal Processing Laboratory Faculty of Sciences and the University of the Algarve Tecnologia (Galician), Version, 1, 2008.

[42] S.Anandalatchoumy and G.Sivaradje. Comprehensive study of acoustic channel models for underwater wireless communication networks. International Journal on Cybernetics and Informatics, 4(2):227-240, April 2015.

[43] W. Seong. Hybrid Galerkin Boundary Element-Wavenurnber Integration Method. PhD thesis, Massachusetts Institute of Technology, 1991.

[44] M. Sipser. Introduction to the Theory of Computation. Cengage Learning, Boston, MA, USA, 3rd edition, 2012.

[45] A. Song, J. Senne, M. Badiey, and K. B. Smith. Underwater acoustic communication channel simulation using parabolic equation. In Proceedings of the Sixth ACM International Workshop on Underwater Networks, page 2. ACM, 2011.

[46] H. P. Stern and S. Mahmoud. Communication systems: analysis and design. Prentice-Hall, Inc., 2003.

[47] M. Stojanovic. Underwater acoustic communications. In Electro/95 International. Professional Program Proceedings., pages 435-440. IEEE, 1995.

[48] M. Stojanovic. On the relationship between capacity and distance in an underwater acoustic communication channel. ACM SIGMOBILE Mobile Computing and Communications Review, 11(4):34-43, 2007.

[49] M. Stojanovic, J. Catipovic, J. G. Proakis, et al. Phase-coherent digital communications for underwater acoustic channels. Oceanic Engineering, IEEE Journal of, 19(1):100-111, 1994.

[50] M. Stojanovic and J. Preisig. Underwater acoustic communication channels: Propagation models and statistical characterization. Communications Magazine, IEEE, 47(1):84-89, 2009.

[51] F. D. Tappert. The parabolic approximation method. In Wave propagation and underwater acoustics, pages 224-287. Springer, 1977. 
[52] Teledyne Benthos. ATM-900 Series Acoustic Telemetry Modems Users Manual P/N M-270-26, Rev. E. Teledyne Benthos, 49 Edgerton Drive, North Falmouth, MA 02556 U.S.A., December 2014.

[53] Teledyne Benthos. Benthos Acoustic Modems. teledynebenthos.com, December 2014.

[54] D. Thomson and N. Chapman. A wide-angle split-step algorithm for the parabolic equation. The Journal of the Acoustical Society of America, 74(6):1848-1854, 1983.

[55] P. van Walree, R. Otnes, et al. Ultrawideband underwater acoustic communication channels. Oceanic Engineering, IEEE Journal of, 38(4):678-688, 2013.

[56] J. Wang, H. Wang, R. Zhang, and F. Li. Chaotic modulations for digital underwater acoustic communications. In Proceedings of the Eighth ACM International Conference on Underwater Networks and Systems, page 10. ACM, 2013.

[57] Wikipedia. Frequency-shift keying. https://en.wikipedia.org/wiki/ Frequency-shift_keying, May 2015.

[58] G. S. Wong and S.-m. Zhu. Speed of sound in seawater as a function of salinity, temperature, and pressure. The Journal of the Acoustical Society of America, 97(3):1732-1736, 1995.

[59] F. Xiong. Digital Modulation Techniques, (Artech House Telecommunications Library). Artech House, Inc., 2006.

[60] W.-B. Yang and T. Yang. High-frequency channel characterization for m-ary frequency-shift-keying underwater acoustic communications. The Journal of the Acoustical Society of America, 120(5):2615-2626, 2006. 